
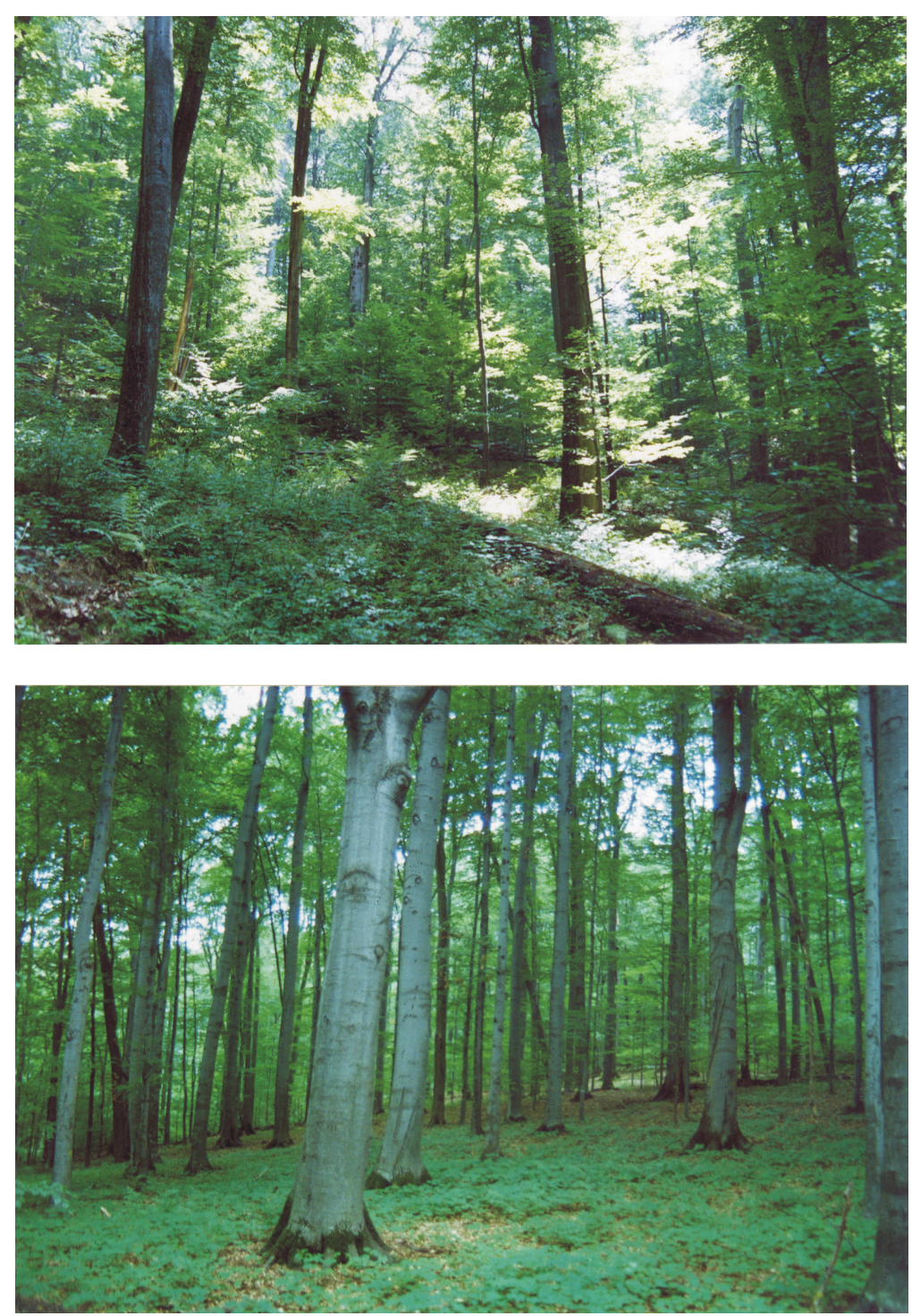

\title{
Struktur und Dynamik von zwei Buchenurwäldern in der Slowakei
}

\author{
Dissertation \\ zur Erlangung des Doktorgrades \\ der Fakultät für Forstwissenschaften und Waldökologie \\ der Georg-August-Universität Göttingen \\ vorgelegt von \\ Lars Drößler \\ Göttingen 2006
}




\section{Inhaltsverzeichnis}

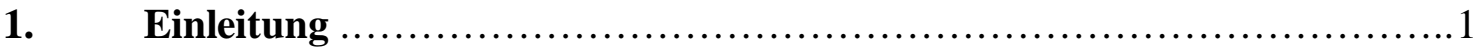

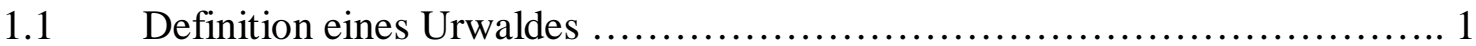

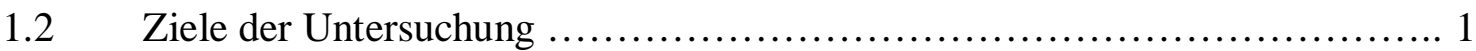

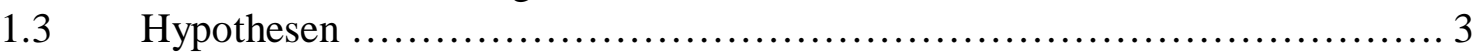

\section{Standortfaktoren, Verjüngung und waldwachstumskundliche}

Kennwerte ............................................................. 5

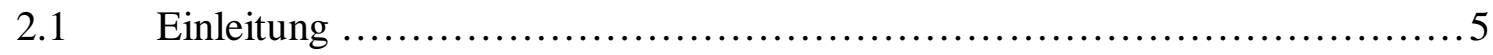

$2.2 \quad$ Material und Methoden ................................................ 5

2.2.1 Beschreibung der Standorte .......................................... 5

2.2.2 Methodik ............................................................. 6

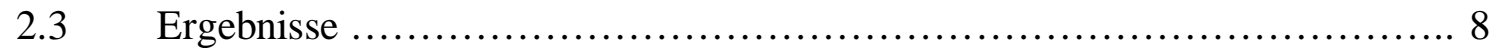

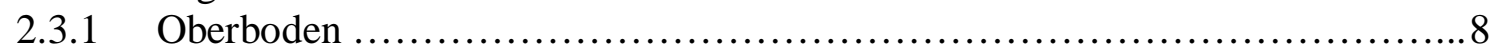

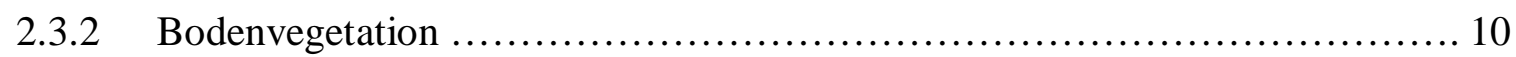

2.3.3 Verjüngung und Strahlungsverhältnisse ................................. 11

2.3.3.1 Dichte, Höhenstruktur und Verbiss ......................................... 11

2.3.3.2 Strahlungsverhältnisse .............................................. 13

2.3.4 Austriebszeitpunkt und jährlicher Jahrestrieb im Vergleich zu Buchen aus

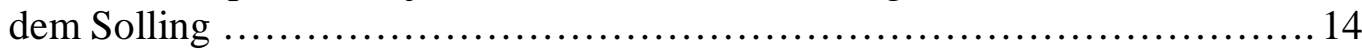

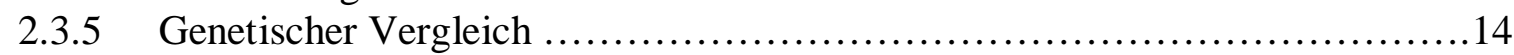

2.3.6 Bestandescharakteristik ................................................ 16

2.3.6.1 Vorrat des lebenden Bestandes ........................................... 16

2.3.6.2 Totholzvorrat............................................................... 17

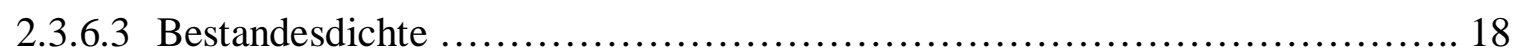

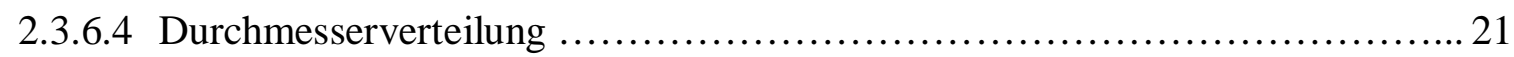

2.3.6.5 Baumhöhen und Kronendimension ....................................... 22

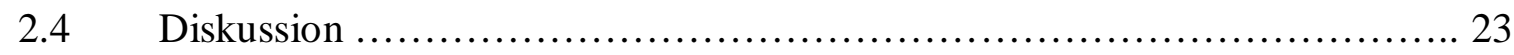

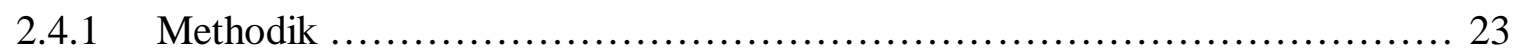

2.4.2 Boden, Krautvegetation und Verjüngung ................................. 24

2.4.3 Die Vergleichbarkeit mit Buchenbeständen in Deutschland ..................... 27

2.4.4 Holzvorrat und Bestandesdichte ....................................... 28

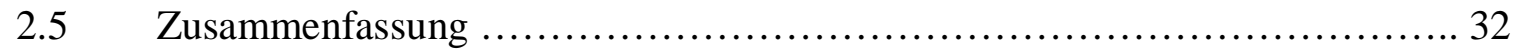

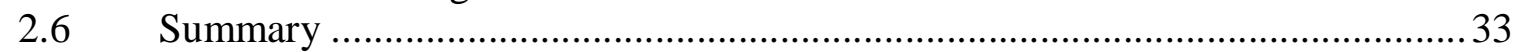

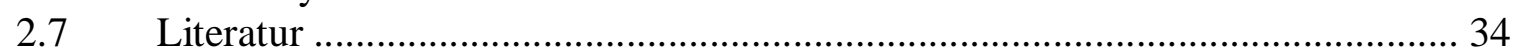

3. Stand structure in virgin and managed beech forests ................................. 37

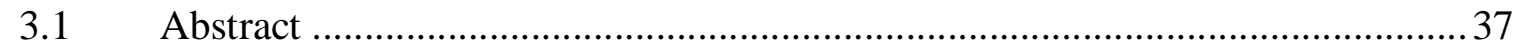

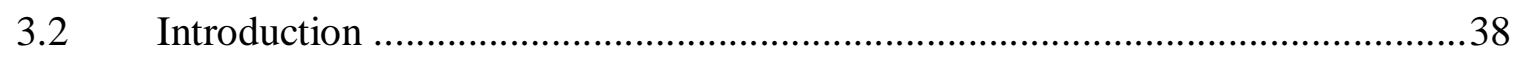

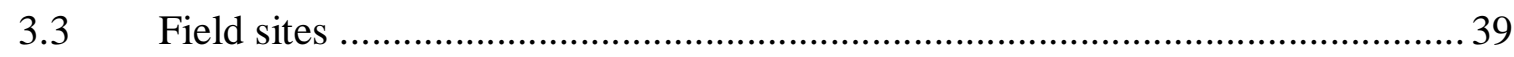

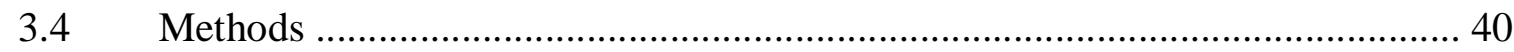

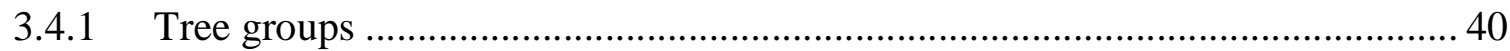

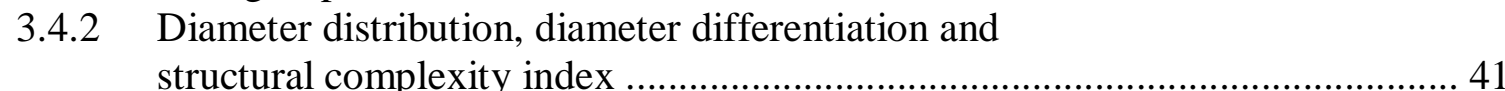

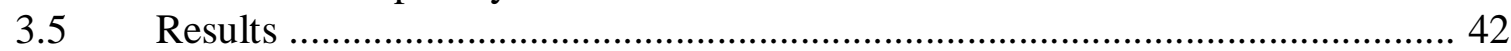

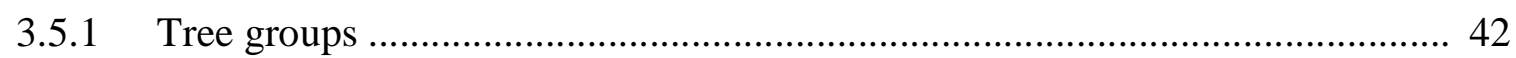

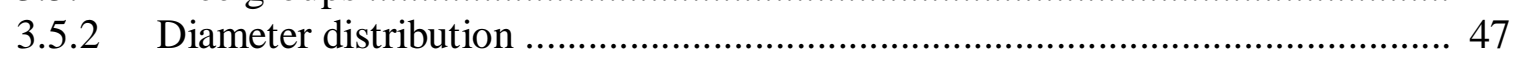

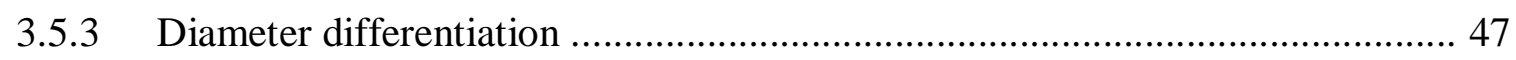

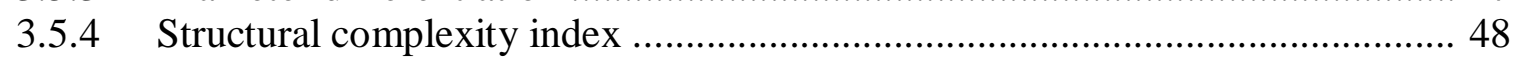

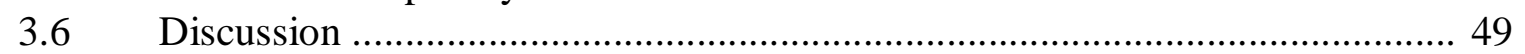




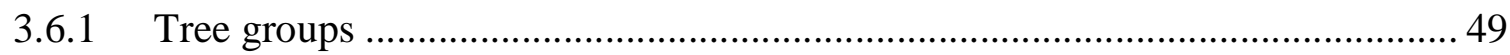

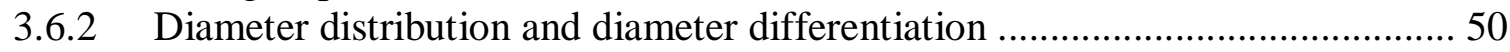

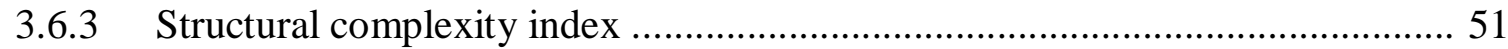

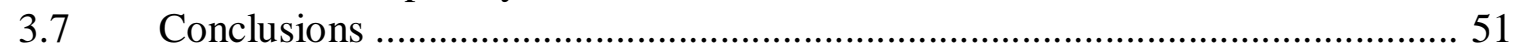

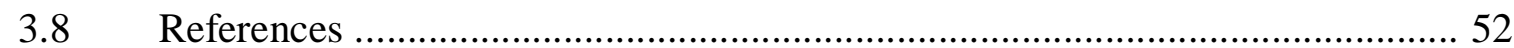

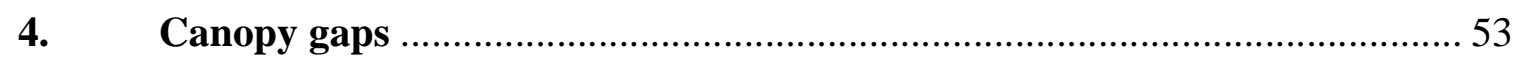

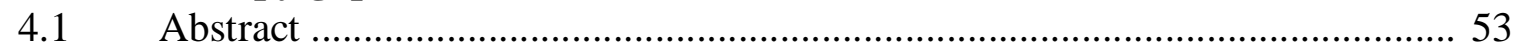

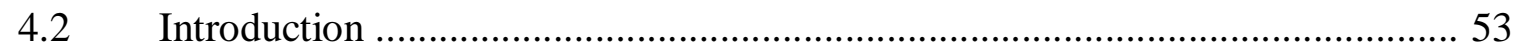

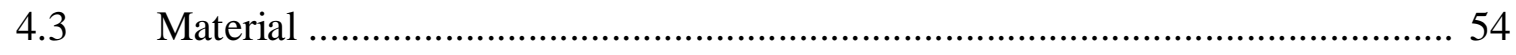

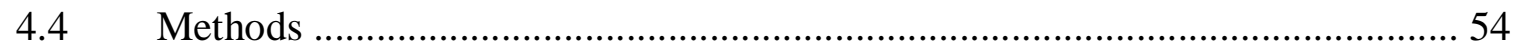

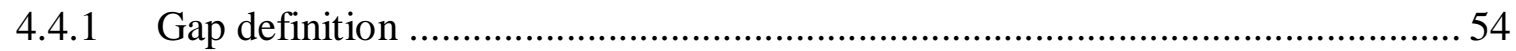

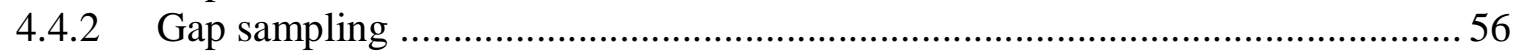

4.4.3 Representative percentage of different sized gaps .........................................56

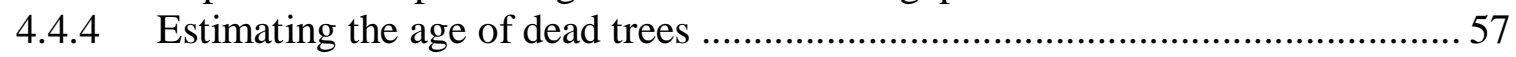

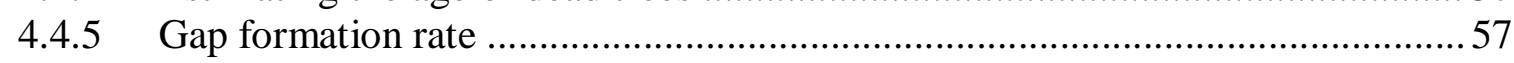

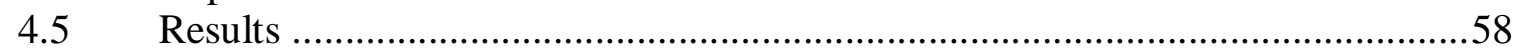

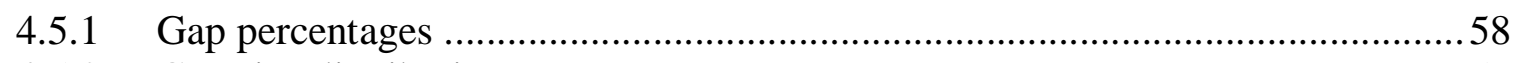

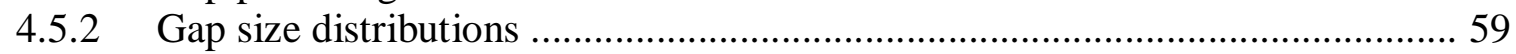

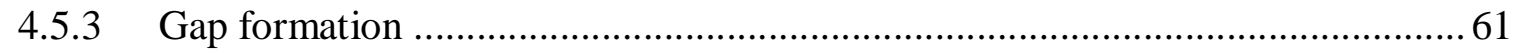

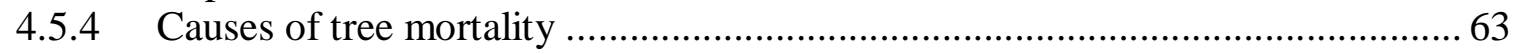

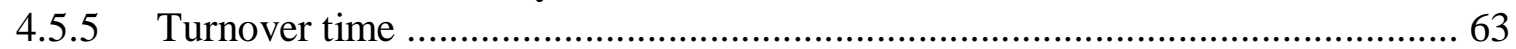

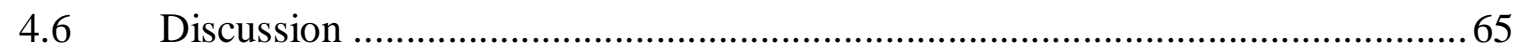

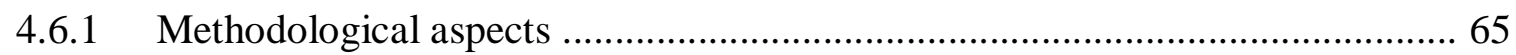

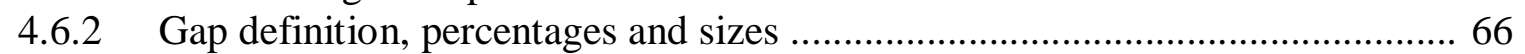

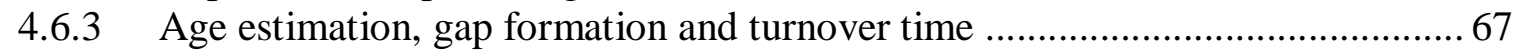

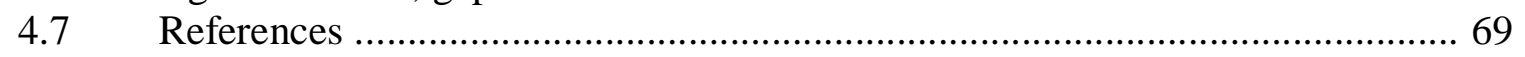

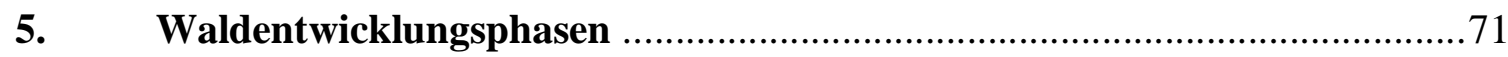

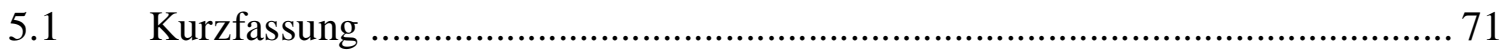

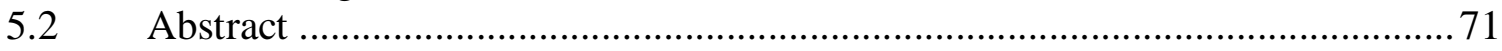

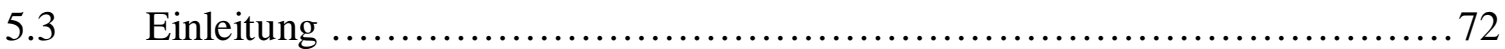

5.4 Material und Methoden .................................................. 72

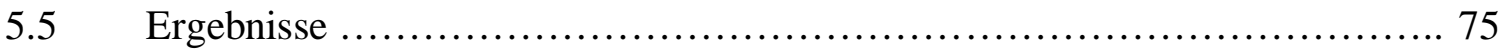

5.5.1 Waldentwicklungsphasen nach dem Verfahren von Tabaku (1999) ............. 75

5.5.2 Die Gruppierung von Bäumen unterschiedlicher Altersstufen ................. 80

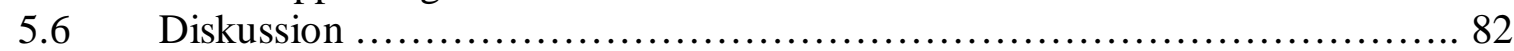

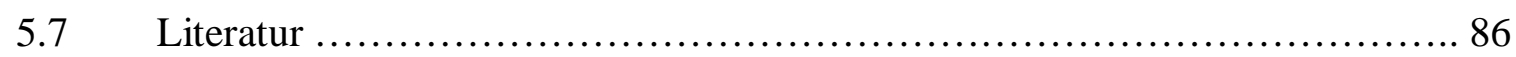

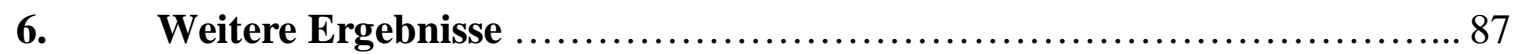

6.1 Paar- und Markenkorrelationsfunktionen .................................. 87

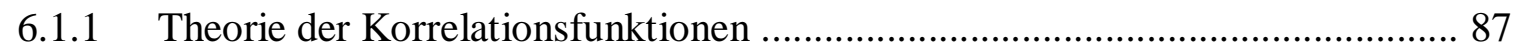

6.1.2 Ergebnisse und Diskussion der Korrelationsfunktionen ................... 88

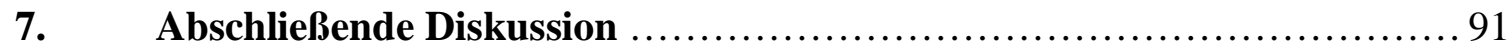

$7.1 \quad$ Diskussion der Hypothesen ............................................... 91

7.2 Waldbauliche Schlussfolgerungen ..................................... 96

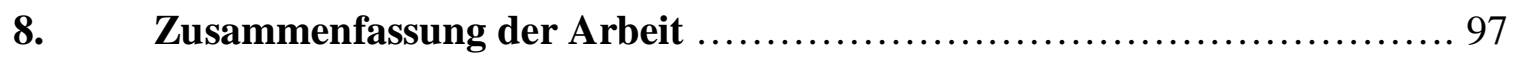

9. Weitere Literatur .......................................................... 100

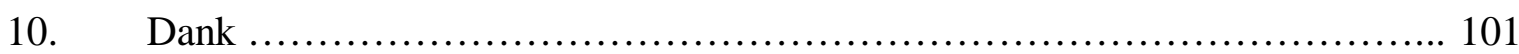




\section{Einleitung}

1.1 Definition eines Urwaldes

Im Folgenden wird Urwald als Wald definiert, in dem noch keine Holznutzung durch Menschen stattgefunden hat. Definitionen anderer Autoren sind in Tabelle 1 aufgeführt. Die in dieser Arbeit verwendete Definition stellt die Mindestanforderung aller Autoren an einen Urwald dar.

Tab. 1 Die Definitionen für einen Urwald durch verschiedene Autoren

\begin{tabular}{|c|c|}
\hline Autor & Definition \\
\hline Cermak (1910) & $\begin{array}{l}\text { Wald, der sich noch vollkommen in dem Zustand befindet, den er ohne } \\
\text { jeden menschlichen Eingriff angenommen hat. }\end{array}$ \\
\hline Rubner (1960) & $\begin{array}{l}\text { Waldvegetation, die hinsichtlich Artenzusammensetzung, Aufbau und } \\
\text { Wuchs als klimatisch bedingtes Schlussglied auftritt. }\end{array}$ \\
\hline Leibundgut (1993) & $\begin{array}{l}\text { Ein vom Menschen unberührter Wald mit riesigen Bäumen, großen } \\
\text { Holzvorräten, einem schwer begehbaren Unterholz und vielen stehenden } \\
\text { und liegenden Baumleichen. }\end{array}$ \\
\hline Korpel' (1995) & $\begin{array}{l}\text { Waldgesellschaft, deren Zusammensetzung, Aufbau, Wachstum und } \\
\text { andere Lebensprozesse durch Eigenschaften der Umwelt, vor allem des } \\
\text { Klimas, bedingt sind. }\end{array}$ \\
\hline
\end{tabular}

Es ist unwahrscheinlich, dass es in Europa Wälder gibt, die noch keinem menschlichen Einfluss unterliegen. Neben Einträgen über die Atmosphäre wurden bereits vor mehreren Jahrhunderten die meisten Wälder als Waldweide oder zur Jagd genutzt. Diese Form der Nutzung wurde mit hoher Wahrscheinlichkeit auch in den beiden vorgestellten Reservaten mit geringer Intensität durchgeführt.

\subsection{Ziele der Untersuchung}

Urwälder sind wichtige Referenzflächen für die Erforschung natürlicher Strukturen und Prozesse. Durch Vergleiche zwischen Urwald und Wirtschaftswald erhält man eine Vorstellung, wie weit man forstlich in Waldökosysteme eingreift. Eine wachsende Bedeutung kommt Urwäldern zu, um globale Stoffflüsse und Stoffvorräte mit quasi-natürlichen Zuständen zu vergleichen (z. Bsp. Kohlenstoffhaushalt). Das Feststellen von Tier- und Pflanzenarten und das Aufstellen von Beziehungen zu ihren Habitaten ist ein weiterer Grund für unser Interesse. Diese angedeuteten Fragestellungen sind nicht Ziel, aber Forschungsumfeld der vorliegenden Arbeit.

Die Arbeit beschreibt den Waldaufbau und das Störungsregime von Buchenurwäldern (Fagus sylvatica) exemplarisch anhand von zwei Reservaten in der Slowakei. Sie konzentriert sich 
auf lebende und tote Bäume als das herausragende Strukturelement im Wald. So betonen Meyer et al. (2001), dass die Waldtextur in Form junger, alter und toter Bäume ein Gerüst für andere Elemente des Waldes darstellen kann. Auf so einer Grundlage kann eine Einordnung häufiger und seltener Struktureinheiten erfolgen, auf die weitergehende Untersuchungen unterschiedlicher wissenschaftlicher Disziplinen Bezug nehmen können.

Die ausgewählten Reservate stellen aus deutscher Sicht die nächstgelegenen ungenutzten Buchenwälder der montanen Höhenstufe dar. Buchen-Urwaldreservate kommen auch in der Ukraine, in Rumänien, Albanien, Kroatien und Slowenien vor. In den slowakischen Reservaten wurden vor ungefähr 50 Jahren Dauerversuchsflächen angelegt und im zehnjährigen Intervall dokumentiert (Korpel' 1995). Eine andere wichtige Untersuchung wurde von Tabaku (1999) in albanischen Urwäldern durchgeführt. Tabaku u. Meyer (1999) sowie Zeibig et al. (2005) liefern Beschreibungen zum Störungsregime in Buchenurwäldern Südosteuropas. Untersuchungen von Neumann (1979), Tsuryk (1980), Leibundgut (1993), Chernyavskyy (2005) und Commarmot et al. (2005) geben ertragskundliche Werte und Waldentwicklungsphasen wieder. Einen interessanten Beitrag über die Alterstruktur einzelner Buchen liefert Korpel' (1967). Frühe Untersuchungen in Ost- und Südosteuropa von Cermak (1910), Fröhlich (1925), Dengler und Markgraf (1931) oder Mauve (1931) beschreiben gutachterlich den Bestandesaufbau von Buchenurwäldern.

Alle genannten Arbeiten haben das Ziel, den Aufbau in Buchenurwäldern zu beschreiben und Entwicklungen zu erkennen. Oft werden auch Schlussfolgerungen für eine naturnahe Waldbewirtschaftung abgeleitet. Dieses Ziel wird auch mit der vorliegenden Arbeit verfolgt. Im Einzelnen stellen sich dazu folgende Teilziele heraus:

- $\quad$ Einschätzung der Vergleichbarkeit slowakischer und deutscher BuchenwaldStandorte (Boden- und Vegetationsansprache, genetische Analyse)

- $\quad$ Ermittlung von Holz- und Totholzvorräten

- $\quad$ Untersuchung der ökologischen Bedingungen für die Verjüngung und Beurteilung der Konkurrenzfähigkeit von Mischbaumarten in diesem Alter

- $\quad$ Quantitative Aussagen zur Anordnung unterschiedlich großer Bäume

- $\quad$ Ableitung des vorherrschenden Störungsregimes aus Strukturbefunden wie Lückengrößen und Lückenbildungsraten 
- $\quad$ Erfassung wichtiger Strukturparameter und Ausscheidung von

Entwicklungsphasen auf der gesamten Fläche

- $\quad$ Vergleich der nach quantitativen Merkmalen ausgeschiedenen

Entwicklungsphasen mit den von Korpel' (1995) ausgeschiedenen Phasen

\subsection{Hypothesen}

In Deutschland haben in allen Buchenwäldern bereits Holznutzungen stattgefunden. Ohne den Einfluss der Menschen würden Buchenurwälder auf $80 \%$ der Fläche Deutschlands vorkommen (Bohn et al. 2003). Tatsächlich nehmen die Wälder heute ein Drittel der Landesfläche ein, werden bewirtschaftet und setzen sich zum Teil aus anderen Baumarten zusammen. Die Bewirtschaftung wird nach strengen Kriterien beurteilt. Waldbau-Programme der Landesforstverwaltungen fordern unter anderem Naturnähe. Urwälder stellen die maximal erreichbare Naturnähe dar, an der sich Beurteilungen orientieren können.

Eine Folge der Forderung nach Naturnähe sind die bereits im großen Maßstab durchgeführte einzelstammweise Nutzung zielstarker Bäume und oft auch eine angestrebte Überführung in ungleichaltrige Wälder. Derartige Veränderungen in der Bewirtschaftung haben zum Teil beträchtliche Investitionen und das Aufgeben gesicherter Erkenntnisse im Altersklassenwald über die Entwicklung von 1-2 Bestandesgenerationen zur Folge. Demgegenüber steht ein vergleichsweise geringes gesichertes Wissen über den Aufbau ungleichaltriger und besonders naturnaher Wälder. Wie sich Bäume ähnlicher Größe im Urwald verteilen und in welchen Mustern die Bäume der Oberschicht absterben, kann zum Beispiel nur vermutet werden. Aus diesem Grund sollen folgende Hypothesen in zwei Buchen-Urwaldreservaten überprüft werden:

I. Die Struktur ist auf kleiner Fläche sehr heterogen. Die Bäume sterben aber nicht nur einzeln ab.

Die heterogene Struktur haben bereits Leibundgut (1993) und Korpel' (1995) angenommen. Im Folgenden soll geprüft werden, ob die Durchmesserdifferenzierung der Bäume deutlich größer ist als im Wirtschaftswald. Zusätzlich soll die maximale Gruppengröße von Bäumen einer Alterstufe festgestellt werden und geprüft werden, ob Bäume mit mehr als $80 \mathrm{~cm}$ BHD nur einzeln stehend auftreten.

Dass Bäume nicht nur einzeln absterben, hat sich bereits für ein Buchenurwaldreservat in Slowenien bestätigt (Zeibig et al. 2005). Zur Überprüfung in den slowakischen 
Reservaten sollen Größenverteilungen der Bestandeslücken erstellt werden. Zusätzlich wird der Frage nachgegangen, ob sich Lücken erweitern.

II. Der Totholzvorrat beträgt weniger als $10 \%$ des Gesamtholzvolumens (lebender Bestand und Totholz).

Diese Vermutung hat sich bereits für albanische Buchenurwälder bestätigt (Tabaku 1999). Die Differenz zu vorgeschlagenen Richtwerten von 15 bis 40 m³/ha (Ammer 1991, Müller 2005) soll ermittelt werden.

III. Verjüngung ist überall vorhanden.

Diese Vermutung hat sich bereits für albanische Buchenurwälder bestätigt (Tabaku 1999). Weiterhin soll geprüft werden, ob neben der Buchenverjüngung ein Anflug anderer Baumarten auf ganzer Fläche erfolgt.

IV. Die ökologischen Bedingungen für das Wachstum der Buchen in der Slowakei sind mit deutschen Buchenbeständen vergleichbar.

Dazu soll der Standort, die Bodenvegetation und die genetische Zusammensetzung von Bucheckern untersucht und mit deutschen Beständen verglichen werden

V. Waldentwicklungsphasen treten meistens deutlich kleinflächiger auf, als auf den von Korpel' (1995) ausgewiesenen Untersuchungsflächen.

Die folgenden Abschnitte 2 bis 3 entsprechen Publikationen, die bei wissenschaftlichen Zeitschriften zur Begutachtung eingereicht wurden. Abschnitt 4 ist bereits im Journal of Forest Science unter dem Titel „Canopy gaps in two virgin beech forest reserves in Slovakia“ und Abschnitt 5 im Forstarchiv unter dem Titel „Waldentwicklungsphasen in zwei BuchenUrwaldreservaten in der Slowakei“ erschienen. Der Text in diesen Abschnitten entspricht den Publikationen. Die Nummern der Überschriften wurden jedoch geändert und Verweise auf andere Abschnitte dieser Arbeit konkretisiert.

Weitere Ergebnisse werden im Anschluss an die Publikationen aufgeführt. Im letzten Teil werden die Hypothesen diskutiert, waldbauliche Schlussfolgerungen gezogen und die wichtigsten Ergebnisse zusammengefasst. 


\section{Standortfaktoren, Verjüngung und waldwachstumskundliche Kennwerte}

Dieser Teil entspricht einem eingereichten Manuskript für die Allgemeine Forst- $u$.

Jagdzeitung mit dem Titel „Bestandesstruktur, Verjüngung und Standortfaktoren in zwei

Buchenurwald-Reservaten der Slowakei“ von L. Drößler und B. v. Lüpke

\section{SCHLAGWÖRTER - KEY WORDS}

Fagus sylvatica; Urwald; Holzvorrat; Bestandesstruktur; Totholz; Verjüngung; Slowakei. Fagus sylvatica; virgin forest; standing volume; stand structure; dead wood; regeneration; Slovakia.

\subsection{EINLEITUNG}

Im Zuge der Gestaltung naturnaher Wälder in der Forstwirtschaft rücken Urwälder verstärkt in den Fokus der Forschung. In Deutschland wären ohne menschlichen Einfluss 80 Prozent der Fläche mit Buchenwald bedeckt (Bohn et al. 2003). Für die Untersuchung wurden deshalb zwei Buchen-Urwaldreservate in der Slowakei ausgewählt. Nach unserer Recherche handelt es sich um die zu Deutschland nächstgelegenen Buchenurwälder unter $800 \mathrm{~m}$ ü. NN.

Ein wichtiges Ziel der Untersuchung war es, Holzvorräte zu bestimmen. Unter anderem wurde der Frage nachgegangen, ob Holzvorräte von $1000 \mathrm{~m}^{3} /$ ha im Urwald überschritten werden und auf welche Flächengröße diese Extrema beschränkt sind. Totholzvorräte wurden ebenso bestimmt. Ein anderes Ziel war die Einschätzung der Wuchsbedingungen in den Untersuchungsbeständen anhand waldökologischer Kenngrößen wie Standort, Bodenvegetation, Strahlung und Verjüngung.

In beiden Urwaldreservaten wurden bereits andere Untersuchungen durchgeführt. Sie betreffen Lücken im Kronendach (Drößler u. Lüpke 2005) und Waldentwicklungsphasen (Drößler u. Meyer 2006).

\subsection{MATERIAL UND METHODEN}

\subsubsection{Beschreibung der Standorte}

Die beiden untersuchten Urwaldbestände befinden sich im Osten der Slowakei. Das Reservat Havešová liegt in den Bergen von Bukov in einer Höhe von 500-650 m ü. NN. Es ist zum Süden exponiert und umfasst eine Fläche von 170 ha. Der jährliche Niederschlag schwankt zwischen 700-800 mm (450 mm in der Vegetationsperiode). Das Klima ist subozeanisch und kontinentaler als in Deutschland. Die mittlere Jahrestemperatur beträgt $7{ }^{\circ} \mathrm{C}$, die Schwankungsbreite der Monatsmittel erreicht ca. $20^{\circ} \mathrm{C}$. Das geologische Ausgangssubstrat 
bilden Meeresablagerungen aus dem späten Eozön in Form von Flysch (Sandstein und Mergel, z.T. mit eingelagerten Tonschichten). Darüber hat sich eine Braunerde entwickelt. Das zweite Reservat Kyjov liegt im Vyhorlat-Gebirge (700-820 m ü. NN). Es ist nach Norden geneigt und umfasst 53 ha. Der jährliche Niederschlag liegt bei 750-800 mm und die Jahresdurchschnittstemperatur beträgt $6{ }^{\circ} \mathrm{C}$. Das Ausgangsgestein für den Boden ist Andesit (vulkanisches Ergussgestein). Darüber hat sich ebenfalls eine Braunerde entwickelt. Die Entfernung zwischen den Reservaten beträgt $50 \mathrm{~km}$. Es handelt sich in beiden Fällen um reine Buchenbestände. Beschreibungen des Waldaufbaus finden sich bei Korpel' (1995) und Drößler u. Lüpke (2005).

\subsubsection{Methodik}

Die Aufnahme des Baumbestands wurde 2002 in Havešová und 2003 in Kyjov durchgeführt. Dazu wurden in den Reservaten 22 bzw. 20 systematisch verteilte Stichprobenflächen angelegt. Der Mindestabstand zur Reservatsgrenze betrug 100 m. Die Flächen waren Quadrate mit 62,5 m Kantenlänge, was eine Fläche von rd. 0,39 ha ergibt. Die ungewöhnliche Größe wurde gewählt, um im Rahmen einer anderen Untersuchung (Drößler u. Meyer 2006) Waldentwicklungsphasen auszuscheiden.

Auf den Stichprobenflächen wurden für jeden Baum ab $7 \mathrm{~cm}$ Brusthöhendurchmesser (BHD) die Lagekoordinaten, der BHD und die Baumart bestimmt. An Bäumen ab $40 \mathrm{~cm}$ BHD wurde die äußerlich sichtbare Stammqualität bis $8 \mathrm{~m}$ Höhe nach den in Deutschland üblichen Güteklassen A bis C angesprochen. Totholzobjekte mit mehr als $20 \mathrm{~cm}$ BHD oder $20 \mathrm{~cm}$ Enddurchmesser sowie mehr als 2 m Länge wurden eingemessen und die Durchmesser aufgenommen. Stärker zersetzte Stämme wurden mit visuell geschätzten Volumenzuschlägen versehen.

Das Holzvolumen des lebenden Bestandes wurde mit Hilfe der Volumenfunktion von Petráš und Pajtík (1991) bestimmt. Die dafür erforderlichen Höhen der einzelnen Bäume wurden einer Bestandeshöhenkurve entnommen (Abb. 10 und 11), die je Reservat von 60 gleichmäßig über das Durchmesserspektrum verteilten Bäumen abgeleitet worden war. An diesen Bäumen wurden auch die Kronenansatzhöhe und acht Kronenradien gemessen. Die Radien wurden gemittelt und die Kronenschirmfläche über ein Kronenkreismodell geschätzt.

Anhand von Stammverteilungsplänen und der Baumhöhen wurde der strukturelle Komplexitätsindex nach Zenner u. Hibbs (2000) berechnet. Dazu wurde eine Triangulation durchgeführt, nach der die gesamte Fläche mit sich nicht überlappenden Dreiecken bedeckt ist 
und die Eckpunkte der Dreiecke auf den Stammfußpunkten der einzelnen Bäume liegen. Die Dreiecke werden entsprechend der Höhe der Bäume dreidimensional aufgerichtet. Das Verhältnis des Flächeninhalts dieses Dreiecks zum Flächeninhalt des Dreiecks in der Ebene ergibt bei gleich hohen Bäumen 1 und steigt mit größeren Höhenunterschieden. Der Durchschnitt des Verhältnisses aller Dreieckspaare auf einer Stichprobenfläche ergibt den strukturellen Komplexitätsindex (Zenner u. Hibbs 2000), nach dem die Stichprobenflächen in Abb. 2-7 geordnet sind.

Zur Aufnahme der Verjüngung wurden die Stichprobenflächen in 25 Quadrate mit 12,5 m Kantenlänge geteilt, deren Mitte den Mittelpunkt für $5 \mathrm{~m}^{2}$ große Probekreise darstellte. Die Quadrate wurden auch als Grundeinheit für die Berechnung des Holzvolumens auf kleiner Fläche verwendet. Die Verjüngungsaufnahme wurde im Jahr 2003 in Havešová und 2004 in Kyjov durchgeführt. Es wurden alle Gehölze bis zu einer Stärke von 4 cm BHD aufgenommen. Keimlinge wurden nicht berücksichtigt. Die Pflanzen wurden nach Höhenklassen, Baumart und Verbiss erfasst.

10 Stichprobenflächen wurden je Reservat zufällig ausgewählt, in denen $1 \mathrm{~m}$ über dem Mittelpunkt der Probekreise der Verjüngung insgesamt 250 hemisphärische Fotos zur Strahlungsschätzung nach der Methode von Wagner (1994) aufgenommen wurden. Nach einer anschließenden computergestützten Auswertung der Fotos konnte das diffuse Strahlungsangebot an bewölkten Tagen durch den Diffuse-Site-Factor prozentual im Vergleich zur Freilandhelligkeit geschätzt werden.

Auf fünf zufällig ausgewählten Stichprobenflächen wurden in Havešová nach einer Buchenmast auf jedem Quadrat zehn Bucheckern gesammelt. Je Stichprobenfläche wurden 20-30 Eckern genetisch analysiert, wobei neun Genloci mittels Gelelektrophorese untersucht wurden. Durch die Analyse sollte ein Kriterium für die Vergleichbarkeit mit deutschen Beständen erarbeitet werden. Dazu wurden die entsprechenden Parameter von zwei Buchenbeständen aus dem Solling und dem Harz herangezogen, von denen jeweils 1000-2000 Eckern untersucht wurden.

Zwei weitere Vergleichskriterien boten sich durch den Dickenzuwachs und den Blattaustrieb junger Buchen aus Havešová und einer Provenienz aus dem Solling. Dazu wurden Bucheckern aus Havešová in einem Versuch von Czajkowski u. Bolte (2006) zur Keimung gebracht. An 162 Pflanzen aus Havešová wurde der Dickenzuwachs im zweiten Jahr festgestellt und mit dem Zuwachs von 15 Pflanzen aus dem Solling verglichen. Je Provenienz 
wurde zusätzlich an 31 Pflanzen der Blattaustrieb nach der Methode von Dierschke (1972) bestimmt. Beide Herkünfte wurden in Töpfen im Gewächshaus angebaut und unter kontrollierten Wuchsbedingungen gleich behandelt.

Die Ansprache der Bodenhorizonte erfolgte an einem Profil je Reservat an der Reservatsgrenze. Die Humus- und Bodenproben für die chemische Analyse wurden an jeweils 12 über das gesamte Reservat verteilten Orten entnommen. Je Ort wurden 4 Bohrungen in 1 m Abstand durchgeführt und Mischproben gebildet. Die Ansprache der Bodenvegetation erfolgte je Reservat auf drei repräsentativ ausgewählten Flächen á $625 \mathrm{~m}^{2}$ nach der Methode von Braun-Blanquet (1964).

\subsection{ERGEBNISSE}

\subsubsection{Oberboden}

In Havešová hat sich auf den überwiegend aus Sandstein gebildeten Flysch-Fließerden eine mesotrophe Braunerde entwickelt. Der Boden ist tiefgründig und skeletthaltig (Tab. 1). Mit $\mathrm{pH}$-Werten über 4,4 befindet er sich im Austauscher-Pufferbereich (Tab. 4). Die hohen Schluffanteile des Feinbodens gewährleisten eine gute Wasserversorgung (Tab. 2). Die Humusform ist ein typischer Moder. Das C/N-Verhältnis beträgt in der Humusauflage in Havešová 24 und in Kyjov 27 (Tab. 3), im Mineralboden (bis 40 cm Tiefe) 9 bzw. 10 (Tab. 4). Stickstoff nimmt im Mineralboden einen Anteil von durchschnittlich $1,7 \mathrm{~g} \mathrm{~kg}^{-1}$ Trockenmasse ein. Kohlenstoff besitzt einen Anteil von $14,3 \mathrm{~g} \mathrm{~kg}^{-1}$. Die Konzentration an pflanzenverfügbarem Phosphor beträgt $3 \mathrm{mg} \mathrm{kg}^{-1}$.

In Kyjov befindet sich über dem Andesitgestein ebenfalls eine mesotrophe Braunerde. Der Boden ist skeletthaltig, die Humusform ist Moder (Tab. 1). Der tonige Lehmboden gewährleistet eine gute Wasserversorgung (Tab. 2). Der in Wasser gemessene pH-Wert ist etwas niedriger und das C/N-Verhältnis geringfügig größer als in Havešová (Tab. 3 und 4). Die absoluten Elementgehalte von C und N sind in Kyjov deutlich höher. 1 kg Mineralboden (Trockenmasse) enthält durchschnittlich 5 g Stickstoff und 52 g Kohlenstoff. Der Anteil von pflanzenverfügbarem Phosphor beträgt 3,9 $\mathrm{mg} \mathrm{kg}^{-1}$. Die Stoffkonzentration von Magnesium beträgt $0,2 \mathrm{cmol} \mathrm{kg}^{-1}$ (Tab. 5), was etwa $0,5 \mathrm{~g} \mathrm{~kg}^{-1}$ entspricht.

Die mittlere Basensättigung der beiden Böden liegt bei 60 bzw. knapp $50 \%$. 
Tab. 1. Bodenprofile in Havešová und Kyjov

\begin{tabular}{|c|c|c|c|c|c|}
\hline \multicolumn{2}{|c|}{ Horizont } & \multirow{2}{*}{$\begin{array}{l}\text { Havešová } \\
\text { mäßig verdichtetes Laub }\end{array}$} & \multicolumn{2}{|c|}{ Horizont } & \multirow{2}{*}{$\begin{array}{l}\text { Kyjov } \\
\text { mäßig verdichtetes Laub }\end{array}$} \\
\hline Ol & $0-2 \mathrm{~cm}$ & & $\mathrm{Ol}$ & $0-1,5 \mathrm{~cm}$ & \\
\hline Of & $2-4 \mathrm{~cm}$ & $\begin{array}{l}\text { Laubblätter in verschiedenen Graden der Zersetzung, } \\
\text { zusammenklebend in Platten }\end{array}$ & Of & $1,5-3 \mathrm{~cm}$ & Laubblätter in verscheidenen Graden der Zersetzung \\
\hline $\mathrm{Oh}$ & $4-4,5 \mathrm{~cm}$ & leicht bis mäßig grießiger Humus, unregelmäßig & Oh & $3-3,5 \mathrm{~cm}$ & schwarzbrauner Humus in Griessform \\
\hline A & $4,5-10 \mathrm{~cm}$ & $\begin{array}{l}\text { dunkle Braunerde, gut durchlüftet, mäßig frisch, } \\
5-10 \% \text { Skelettanteil, gut durchwurzelt }\end{array}$ & A & $3,5-9 \mathrm{~cm}$ & $\begin{array}{l}\text { dunkle Braunerde, gut durchlüftet, frisch-feucht, } \\
\text { leicht körnig bis körnig, schwach kieselig, } \\
15 \% \text { Skelettanteil, gut durchwurzelt }\end{array}$ \\
\hline B & $10-65 \mathrm{~cm}$ & $\begin{array}{l}\text { hellbraun-beige, sandige Erde, frisch-feucht, mäßig } \\
\text { verdichtet, } 25 \% \text { Skelettanteil, gut durchwurzelt }\end{array}$ & B & $9-45 \mathrm{~cm}$ & $\begin{array}{l}\text { Braunerde, mäßig verdichtet, frisch-feucht, } \\
\text { leicht körnig bis körnig, schwach kieselig, } \\
10-20 \% \text { Skelettanteil, gut durchwurzelt }\end{array}$ \\
\hline C & $65-100 \mathrm{~cm}$ & $\begin{array}{l}\text { hellbraun-beige, sandige Erde, verdichtet, } \\
\text { frisch-feucht, } 70 \% \text { Skelettanteil, vereinzelt Wurzeln }\end{array}$ & C & $45-80 \mathrm{~cm}$ & $\begin{array}{l}\text { hellbraune, tonige Erde, verdichtet, feucht, verklumpt, } \\
80 \% \text { Skelettanteil, vereinzelt Wurzeln }\end{array}$ \\
\hline
\end{tabular}

Tab. 2. Anteile der Korngrößenfraktionen in 0-40 cm Tiefe des Mineralbodens [\%]

\begin{tabular}{lcccc}
\hline Reservat & Ton & Schluff & Feinsand & Grobsand \\
\hline Havešová & 35,8 & 41,3 & 10,6 & 12,3 \\
Kyjov & 25,2 & 41,9 & 27,6 & 5,3 \\
\hline
\end{tabular}

Tab. 3. Mittlere Elementgehalte in der Humusauflage

\begin{tabular}{lrllllll}
\hline Reservat & $\mathrm{N}$ in \% & $\mathrm{C}$ in \% & $\mathrm{C} / \mathrm{N}$ & $\mathrm{P}$ & $\mathrm{K}$ & $\mathrm{Mg}$ & $\mathrm{Ca}$ \\
& & & \multicolumn{5}{c}{ [g/kg Trockenmasse] } \\
\hline Havešová & 1,62 & 39,1 & 24,43 & 0,95 & 2,26 & 0,83 & 5,07 \\
Kyjov & 1,43 & 38,5 & 27,13 & 0,71 & 3,07 & 0,89 & 6,93 \\
\hline
\end{tabular}

Tab. 4. pH-Werte und Elementgehalte von Kohlenstoff, Stickstoff und pflanzenverfügbarem Phosphor in 0-40 cm Tiefe des Mineralbodens

\begin{tabular}{lccccccc}
\hline Reservat & Tiefe & \multicolumn{2}{c}{$\mathrm{pH}-$ Werte } & $\mathrm{C}$ & $\mathrm{N}$ & $\mathrm{C} / \mathrm{N}$ & $\begin{array}{c}\mathrm{P} \\
{[\mathrm{g} / \mathrm{kg}]}\end{array}$ \\
\hline \multirow{3}{*}{ Havešová } & $10 \mathrm{~cm}$ & 4,42 & 3,57 & 25,96 & 3,00 & 8,69 & 4,83 \\
& $20 \mathrm{~cm}$ & 4,60 & 3,68 & 14,30 & 1,74 & 8,74 & 2,53 \\
& $30 \mathrm{~cm}$ & 4,78 & 3,76 & 10,06 & 1,09 & 9,99 & 2,32 \\
& $40 \mathrm{~cm}$ & 4,94 & 3,84 & 6,71 & 0,80 & 9,22 & 2,12 \\
& & & & & & & \\
Kyjov & $10 \mathrm{~cm}$ & 4,18 & 3,80 & 74,82 & 6,73 & 10,80 & 3,82 \\
& $20 \mathrm{~cm}$ & 4,39 & 4,04 & 55,48 & 5,45 & 10,10 & 3,45 \\
& $30 \mathrm{~cm}$ & 4,55 & 4,15 & 46,52 & 4,43 & 10,49 & 4,20 \\
& $40 \mathrm{~cm}$ & 4,62 & 4,20 & 33,07 & 3,32 & 9,95 & 3,93 \\
\hline
\end{tabular}


Tab. 5. Effektive Kationenaustauschkapazität (AKe) und die Anteile der einzelnen Elemente

\begin{tabular}{lcccccccccc}
\hline Reservat & Tiefe & AKe & \multicolumn{10}{c}{ Anteile an der AKe $[\%]$} & & & \\
& & {$[\mathrm{cmol} / \mathrm{kg}]$} & $\mathrm{Ca}$ & $\mathrm{K}$ & $\mathrm{Mg}$ & $\mathrm{Na}$ & $\mathrm{Fe}$ & $\mathrm{Mn}$ & $\mathrm{Al}$ & $\mathrm{H}$ \\
\hline \multirow{3}{*}{ Havešová } & $10 \mathrm{~cm}$ & 7,34 & 48,59 & 14,13 & 5,45 & 1,43 & 0,10 & 1,79 & 27,54 & 0,97 \\
& $20 \mathrm{~cm}$ & 4,78 & 41,02 & 9,76 & 5,58 & 2,13 & 0,03 & 1,29 & 38,90 & 1,29 \\
& $30 \mathrm{~cm}$ & 4,44 & 37,58 & 9,30 & 7,33 & 2,61 & 0,00 & 1,17 & 40,75 & 1,25 \\
& $40 \mathrm{~cm}$ & 4,22 & 38,31 & 8,29 & 12,83 & 2,58 & 0,00 & 2,24 & 34,68 & 1,07 \\
& & & & & & & & & & \\
Kyjov & $10 \mathrm{~cm}$ & 6,98 & 25,72 & 18,72 & 3,78 & 1,22 & 0,06 & 0,72 & 48,88 & 0,90 \\
& $20 \mathrm{~cm}$ & 5,34 & 27,52 & 10,78 & 3,45 & 1,69 & 0,00 & 0,38 & 55,36 & 0,81 \\
& $30 \mathrm{~cm}$ & 4,51 & 33,93 & 8,84 & 3,72 & 2,17 & 0,00 & 0,33 & 50,13 & 0,88 \\
& $40 \mathrm{~cm}$ & 4,23 & 32,87 & 8,37 & 4,15 & 2,51 & 0,00 & 0,26 & 51,04 & 0,80 \\
\hline
\end{tabular}

\subsubsection{Bodenvegetation}

Die Bodenvegetation ist relativ spärlich ausgebildet und bedeckt weniger als ein Drittel der

Fläche (Tab. 6). Die Artenzahl ist in Havešová deutlich niedriger (6 Arten) als in Kyjov (24 Arten). Die häufigsten Arten waren in Havešová Dentaria bulbifera und Galium odoratum und in Kyjov Rubus hirtus und Dryopteris filix-mas.

Die Zusammensetzung der Krautschicht entspricht in beiden Reservaten dem Dentario glandulosae-Fagetum der herzynisch-karpatischen Buchenwälder (Bohn et al., 2003). Als Nebenbaumarten kamen in der Baum- und Strauchschicht Acer pseudoplatanus, A. platanoides und Fraxinus excelsior in sehr geringen Anteilen von unter einem Prozent der Baumzahl (mit mindestens $7 \mathrm{~cm}$ BHD) vor. 
Tab. 6. Deckungsgrade, Artenzahl und Stetigkeiten der Pflanzenarten nach Braun-Blanquet (1964) auf drei repräsentativen Aufnahmeflächen je Reservat

\begin{tabular}{|c|c|c|c|c|c|c|}
\hline \multirow[b]{2}{*}{ Deckungsgrad Baumschicht (\%) } & \multicolumn{3}{|c|}{ Havešová } & \multicolumn{3}{|c|}{ Kyjov } \\
\hline & 95 & 90 & 100 & 90 & 90 & 95 \\
\hline Deckungsgrad Strauchschicht (\%) & 10 & 5 & 5 & 5 & 10 & 5 \\
\hline Deckungsgrad Krautschicht (\%) & 10 & 30 & 0 & 25 & 20 & 35 \\
\hline Artenzahl & 3 & 5 & 1 & 13 & 12 & 19 \\
\hline \multicolumn{7}{|l|}{ Baumschicht } \\
\hline Fagus sylvatica & 5 & 5 & 5 & 5 & 5 & 5 \\
\hline Acer pseudoplatanus & & 2 & & & & 2 \\
\hline \multicolumn{7}{|l|}{ Strauchschicht } \\
\hline Fagus sylvatica & 1 & 1 & 1 & 2 & 2 & 1 \\
\hline Acer pseudoplatanus & & 1 & & & & 1 \\
\hline Fraxinus excelsior & & & & & & + \\
\hline \multicolumn{7}{|l|}{ Krautschicht* } \\
\hline Dentaria bulbifera & 2 & 2 & & & & + \\
\hline Dentaria glandulosa & 1 & & & 1 & + & 1 \\
\hline Galium odoratum & & 2 & & & & \\
\hline Carex pilosa & & 1 & & & & \\
\hline Rubus hirtus & & + & & 2 & 2 & 2 \\
\hline Rubus idaeus & & + & & & & + \\
\hline Dryopteris filix-mas & & & & 1 & 1 & 2 \\
\hline Athyrium filix-femina & & & & 1 & 1 & 1 \\
\hline Paris quadrifolia & & + & & 1 & + & + \\
\hline Lamiastrum galeobdolon & & & & 1 & + & 1 \\
\hline Prenanthes purpurea & & & & $\mathrm{r}$ & & \\
\hline Polygonatum verticillatum & & & & $r$ & & \\
\hline Anemone nemorosa & & & & + & + & 1 \\
\hline Dryopteris carthusiana & & & & + & + & \\
\hline Maianthemum bifolium & & & & + & & \\
\hline Senecio ovatus & & & & & & 2 \\
\hline Impatiens noli-tangere & & & & & + & $\mathrm{r}$ \\
\hline Dryopteris expansa & & & & & + & \\
\hline Gymnocarpium dryopteris & & & & & & + \\
\hline Urtica dioica & & & & & & 1 \\
\hline Galeopsis speciosa & & & & & & + \\
\hline Lunaria rediviva & & & & & & + \\
\hline Salvia glutinosa & & & & & & + \\
\hline
\end{tabular}

* Informationen zu den Baumarten in der Krautschicht finden sich unter 2.3.3.1

\subsubsection{Die Verjüngung und Strahlungsverhältnisse}

\subsubsection{Dichte, Höhenstruktur und Verbiss}

Die Verjüngungsdichte lag bei 65.000 bzw. 77.000 Individuen pro Hektar, wobei mehr als die Hälfte der Pflanzen kleiner als $20 \mathrm{~cm}$ war. Tab. 7 zeigt, dass der Anteil an Mischbaumarten bis zu dieser Größe sehr hoch ist, obwohl der Hauptbestand fast ausschließlich aus Buchen besteht. Ab 1 m Höhe besteht die Verjüngung zu 95-100 \% aus Buche. Neben der Buche 
waren Bergahorn, Spitzahorn und Esche vertreten. Vereinzelt kamen Bergulme, Hainbuche, Haselnuss, Saalweide, Birke und Holunder vor. In Kyjov trat zusätzlich die Eberesche auf. Der Anteil der Mischbaumarten in der Verjüngung betrug in Havešová knapp 50 \% und in Kyjov $10 \%$.

Tab. 8 stellt Unterschiede bei der Besetzung der Probekreise heraus. Mehr als $90 \%$ aller Probekreise waren mit Jungpflanzen besetzt. Aber nur $40 \%$ der Probekreise waren mit Pflanzen besetzt, die höher als ein Meter waren. Die Dichte der mehr als ein Meter hohen Pflanzen betrug in beiden Reservaten rd. 3400 Individuen je Hektar. Die Streuung der Dichte war aber groß: Der Variationskoeffizient betrug in Havešová $243 \%$ und in Kyjov $342 \%$.

Tab. 7. Dichte und Höhenstruktur der Verjüngung mit Anteil der Buche je Höhenklasse

\begin{tabular}{|c|c|c|c|c|c|c|c|c|c|}
\hline \multirow[t]{2}{*}{$\overline{\text { Reservat }}$} & \multirow{2}{*}{$\begin{array}{c}\text { Anzahl } \\
\text { Probekreise } \\
\end{array}$} & \multirow[t]{2}{*}{$\mathrm{N} / \mathrm{ha}$} & \multicolumn{7}{|c|}{ Höhenverteilung [\% von N] } \\
\hline & & & $<0,2$ & $0,2-0,5$ & $0,5-1$ & $1-2$ & $2-3$ & $3-4$ & $>4 \mathrm{~m}$ \\
\hline $\begin{array}{l}\text { Havešová } \\
\text { (Buche) }\end{array}$ & 550 & $\begin{array}{l}64567 \\
(51,7)\end{array}$ & $\begin{array}{l}55,02 \\
(40,9)\end{array}$ & $\begin{array}{l}32,20 \\
(56,1)\end{array}$ & $\begin{array}{c}7,54 \\
(81,1)\end{array}$ & $\begin{array}{c}3,37 \\
(95,0)\end{array}$ & $\begin{array}{c}0,88 \\
(95,5)\end{array}$ & $\begin{array}{c}0,47 \\
(100)\end{array}$ & $\begin{array}{c}0,46 \\
(100)\end{array}$ \\
\hline $\begin{array}{l}\text { Kyjov } \\
\text { (Buche) }\end{array}$ & 500 & $\begin{array}{l}77292 \\
(89,4) \\
\end{array}$ & $\begin{array}{l}73,91 \\
(90,8) \\
\end{array}$ & $\begin{array}{l}13,56 \\
(77,3) \\
\end{array}$ & $\begin{array}{r}7,72 \\
(91,2) \\
\end{array}$ & $\begin{array}{r}4,07 \\
(98,5) \\
\end{array}$ & $\begin{array}{r}0,56 \\
(100) \\
\end{array}$ & $\begin{array}{c}0,14 \\
(100) \\
\end{array}$ & $\begin{array}{c}0,04 \\
(100) \\
\end{array}$ \\
\hline
\end{tabular}

Tab. 8. Statistische Kennwerte der Verjüngungsdichte auf den $5 \mathrm{~m}^{2}$-Probekreisen (Pflanzenzahl je Hektar)

\begin{tabular}{lcccc}
\hline & \multicolumn{2}{c}{ Havešová } & \multicolumn{2}{c}{ Kyjov } \\
& $\begin{array}{c}\text { alle } \\
\text { Jungpflanzen }\end{array}$ & $\begin{array}{c}\text { Jungpflanzen } \\
\text { ab 1m Höhe }\end{array}$ & $\begin{array}{c}\text { alle } \\
\text { Jungpflanzen }\end{array}$ & $\begin{array}{c}\text { Jungpflanzen } \\
\text { ab 1m Höhe }\end{array}$ \\
\hline Mittelwert & 64567 & 3215 & 77292 & 3504 \\
Standardabweichung & 95021 & 7814 & 92311 & 11990 \\
Minimum & 0 & 0 & 0 & 0 \\
Maximum & 714000 & 88000 & 914000 & 190000 \\
Anzahl der Probekreise & 550 & 550 & 500 & 500 \\
davon unbesetzte Kreise & 20 & 325 & 36 & 315 \\
Median & 29000 & 0 & 51000 & 0 \\
\hline
\end{tabular}

In Havešová waren 0,03 \% aller Buchen und 0,3\% aller Nebenbaumarten im Jahr der Aufnahme verbissen. In Kyjov betrug der Anteil 0,01 \% bei Buchen und 0,05\% bei den anderen Baumarten. 90 \% der beschädigten Pflanzen waren 0,2 bis $1 \mathrm{~m}$ hoch. 


\subsubsection{Strahlungsverhältnisse}

Deutliche Unterschiede bestanden zwischen den Reservaten hinsichtlich der Strahlungsverhältnisse am Waldboden. Der Diffuse-Site-Factor betrug in Havešová im Durchschnitt 2,5 \% und in Kyjov 9,4 \%. Die Probepunkte mit Werten unter 5 \% nahmen in Havešová einen Anteil von 85,6 \% und in Kyjov 38,8 \% ein. Der Maximalwert erreichte in Havešová an einem Punkt 38,6 \% und in Kyjov 46,7 \% (Abb. 1).

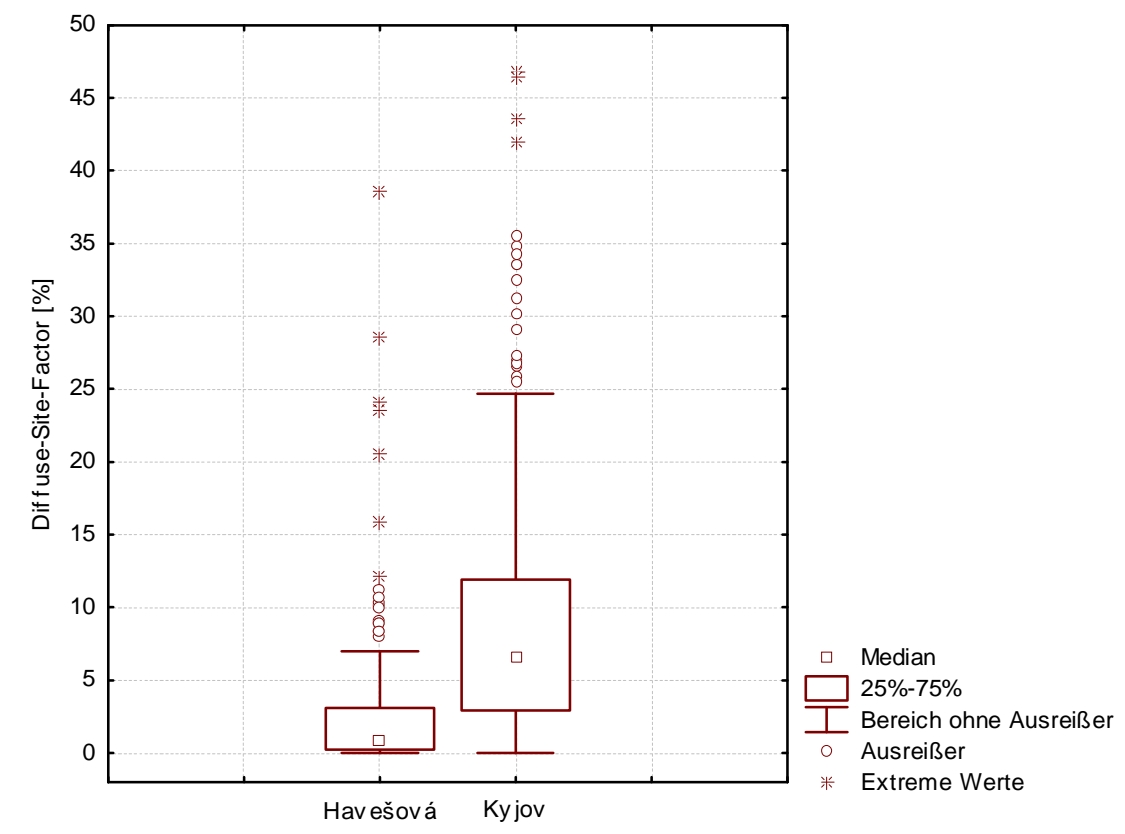

Abb. 1. Boxplot-Diagramm des Diffuse-Site-Factors [\%] in Havešová und Kyjov

Regressionsanalysen zeigten in beiden Reservaten einen signifikanten Zusammenhang zwischen Strahlungsangebot und maximaler Höhe der Verjüngung, wobei der Diffuse-SiteFactor Veränderungen der maximalen Höhe mit Hilfe eines linearen Modells aber nicht erklären konnte $\left(\mathrm{R}^{2}<0,02\right)$. Kein Zusammenhang ergab sich für Regressionen zwischen Strahlungsangebot und der Verjüngungsdichte (sowohl für Buche als auch für Mischbaumarten).

Bei der Verjüngungsaufnahme fiel jedoch auf, dass Mischbaumarten mit weniger als $20 \mathrm{~cm}$ Höhe besonders häufig an dunklen Stellen auftraten. So kamen $71 \%$ der Mischbaumarten in Havešová auf Probekreisen mit einem Diffuse-Site-Factor unter $2 \%$ vor, in Kyjov $28 \%$. Bei einem Diffuse-Site-Factor unter $5 \%$ kamen in Kyjov $59 \%$ der Mischbaumarten vor. 


\subsubsection{Austriebszeitpunkt und jährlicher Dickenzuwachs von Sämlingen aus Havešová- und Solling-Saatgut}

Tab. 9 gibt die Anzahl der Buchen wieder, die nach Knospenzustand unterschieden wurden.

Die Spalten geben fünftägige Intervalle wieder. Jungpflanzen aus der Slowakei begannen rd. 3-4 Tage früher mit dem Austrieb als die Buchen aus dem Solling. Die Beobachtung der Sollingbuchen musste nach dem 16. April abgebrochen werden, da sie in einem anderen Versuch verwendet wurden. Die Buchen aus der Slowakei waren am 21.4. bis zur vollen Blattentfaltung ausgetrieben.

Tab. 9. Austriebszeitpunkt der Buchenblätter zu Beginn der 2. Vegetationsperiode (31 Pflanzen je Herkunft)

\begin{tabular}{lccccccccc}
\hline & \multicolumn{4}{c}{ Havešová } & \multicolumn{4}{c}{ Solling } \\
& 01.04. & 06.04. & 11.04. & 16.04 & 01.04. & 06.04. & 11.04. & 16.04. \\
\hline Winterknospen & 19 & 7 & 2 & - & 30 & 13 & 8 & 2 \\
dicke Knospen & 12 & 15 & 16 & 4 & 1 & 16 & 15 & 8 \\
Knospen mit grünen Spitzen & - & 3 & 5 & 11 & - & 1 & 4 & 7 \\
grüne Blatttüten & - & 6 & 4 & 5 & - & 1 & 3 & 10 \\
$50 \%$ Blattentfaltung & - & - & 4 & 5 & - & - & 1 & 3 \\
volle Blattentfaltung & - & - & - & 6 & - & - & - & 1 \\
\hline
\end{tabular}

Der relative Dickenzuwachs betrug im zweiten Jahr für die Jungpflanzen aus Havešová 50 \%, für die aus dem Solling $40 \%$.

\subsubsection{Genetischer Vergleich}

Die genetische Diversität ist ein Maß, welches auf der Anzahl und dem Verteilungsmuster der Genvarianten basiert. Je größer die Anzahl der Genvarianten ist und je gleichmäßiger diese verteilt sind, umso höher ist die Diversität (Hattemer et al. 1993). Tabelle 10 zeigt nur geringe Unterschiede der Diversität von Buchen aus deutschen Beständen und aus Havešová.

Lediglich die Stichprobe E besaß einen höheren Anteil an Allelen an einem Genlocus. Hierfür können aber Zufallseffekte aufgrund des geringen Stichprobenumfangs eine Rolle spielen. 
Tab. 10. Genetische Diversität von Bucheckern, gesammelt auf fünf Stichprobenflächen in Havešová und in vier deutschen Buchenbeständen

\begin{tabular}{rc}
\hline Genpool & Genetische Diversität \\
\hline Havešová Stichprobe A & 1,423 \\
Havešová Stichprobe B & 1,430 \\
Havešová Stichprobe C & 1,462 \\
Havešová Stichprobe D & 1,467 \\
Havešová Stichprobe E & 1,549 \\
Solling A & 1,472 \\
Solling B & 1,494 \\
Harz A & 1,482 \\
Harz B & 1,511 \\
\hline
\end{tabular}

Der genetische Abstand in Tab. 11 gibt an, wie hoch der relative Anteil am genetischen Material ist, dass nicht mit den Allelen am selben Genlocus übereinstimmt. So müssten für die Bestände aus Deutschland bei 3 bis 5,7 \% der Allele ihre Lage verändert werden, um identisches Genmaterial zu erzeugen. Die Probeflächen in Havešová wiesen untereinander Unterschiede um 5,8 bis 9,4\% auf. Die Unterschiede zwischen Havešová und den deutschen Beständen betrugen 6 bis $11,1 \%$.

Tab. 11. Genetischer Abstand von Bucheckern auf fünf Stichprobenflächen in Havešová und in vier deutschen Buchenbeständen (paarweiser Vergleich)

\begin{tabular}{|c|c|c|c|c|c|c|c|c|c|}
\hline & Hav A & Hav B & Hav C & Hav D & Hav E & Soll A & Soll B & Harz A & Jarz B \\
\hline Hav A & - & & & & & & & & \\
\hline Hav B & 7,6 & - & & & & & & & \\
\hline Hav C & 9,4 & 7,1 & - & & & & & & \\
\hline Hav D & 5,8 & 8,0 & 8,6 & - & & & & & \\
\hline Hav E & 9,0 & 8,3 & 7,5 & 6,7 & - & & & & \\
\hline Soll A & 6,0 & 7,4 & 9,8 & 7,4 & 9,2 & - & & & \\
\hline Soll B & 6,6 & 8,5 & 11,1 & 6,9 & 9,1 & 3,0 & - & & \\
\hline Harz A & 7,5 & 8,2 & 10,6 & 7,8 & 9,3 & 3,3 & 3,2 & - & \\
\hline Harz B & 7,5 & 7,8 & 10,9 & 6,8 & 10,7 & 4,7 & 5,2 & 5,7 & - \\
\hline
\end{tabular}




\subsubsection{Bestandescharakteristik}

\subsubsection{Vorrat des lebenden Bestandes}

Der Derbholzvorrat der lebenden Bäume schwankte in Havešová auf 0,39 ha großen Flächen zwischen 450 und $960 \mathrm{~m}^{3} \mathrm{ha}^{-1}$ (Abb. 2). Der Mittelwert betrug $716 \mathrm{~m}^{3} \mathrm{ha}^{-1}$ (Standardfehler: \pm $25 \mathrm{~m}^{3} \mathrm{ha}^{-1}$ ). In Kyjov war der Derbholzvorrat des lebenden Bestandes deutlich niedriger. Er schwankte zwischen 352 und $553 \mathrm{~m}^{3} \mathrm{ha}^{-1}$ und lag im Durchschnitt bei $451 \mathrm{~m}^{3} \mathrm{ha}^{-1}$ (Abb. 3). Der Standardfehler des Mittelwerts betrug $\pm 12 \mathrm{~m}^{3} \mathrm{ha}^{-1}$.

Für eine kleinflächige Analyse der Holzvorräte wurden die einzelnen Probeflächen jeweils in 25 Quadrate mit je 12,5 m Kantenlänge eingeteilt. Ein besonders hoher Holzvorrat von 1000 m³/ha wurde in Havešová auf insgesamt 17 benachbarten Quadraten mit einer Gesamtfläche von rd. $2650 \mathrm{~m}^{2}$ festgestellt. Ein besonders geringer Vorrat befand sich in einem Fall auf insgesamt 16 benachbarten Quadraten $\left(2500 \mathrm{~m}^{2}\right)$ und betrug $136 \mathrm{~m}^{3} \mathrm{ha}^{-1}$. In Kyjov wurden Holzvorräte von $1000 \mathrm{~m}^{3} \mathrm{ha}^{-1}$ nur auf 3 einzelnen 12,5 x 12,5 m-Quadraten erreicht. Auf 17 benachbarten Quadraten (rd. $2650 \mathrm{~m}^{2}$ ) stockten in einem Fall sehr geringe Holzvorräte von $104 \mathrm{~m}^{3} \mathrm{ha}^{-1}$.

Eine Ansprache der äußerlich sichtbaren Stammqualität von Bäumen ab 40 cm BHD ergab in Havešová, dass 4,7 \% der Bäume eine Einstufung in die in Deutschland handelsübliche Klasse B erlauben, in Kyjov betrug der Anteil 2,6 \%. Der übrige Anteil setzte sich aus Bäumen mit schlechterer Qualität zusammen. Ein Zusammenhang zwischen der Anzahl der Bäume der Klasse B und der Bestandesdichte der einzelnen Probeflächen wurde nicht festgestellt.

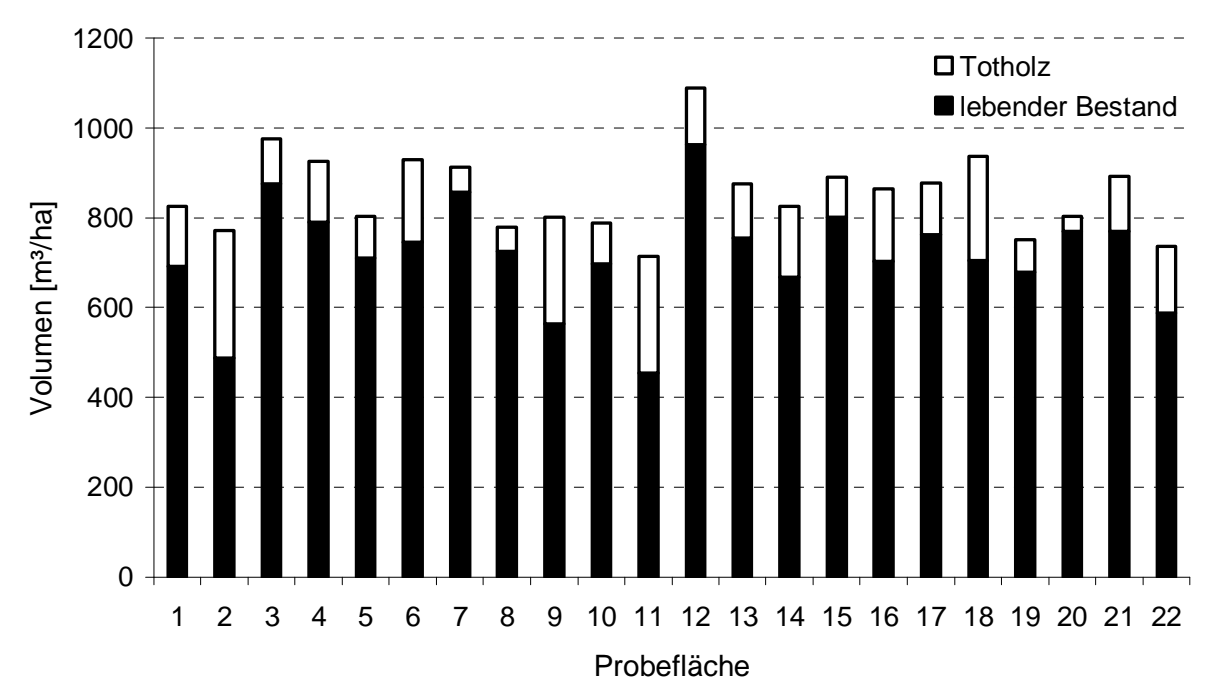

Abb. 2. Derbholzvorrat (lebend und tot) auf 22 Probeflächen in Havešová (Die Strukturvielfalt im Bestandesaufbau nimmt nach rechts zu.) 


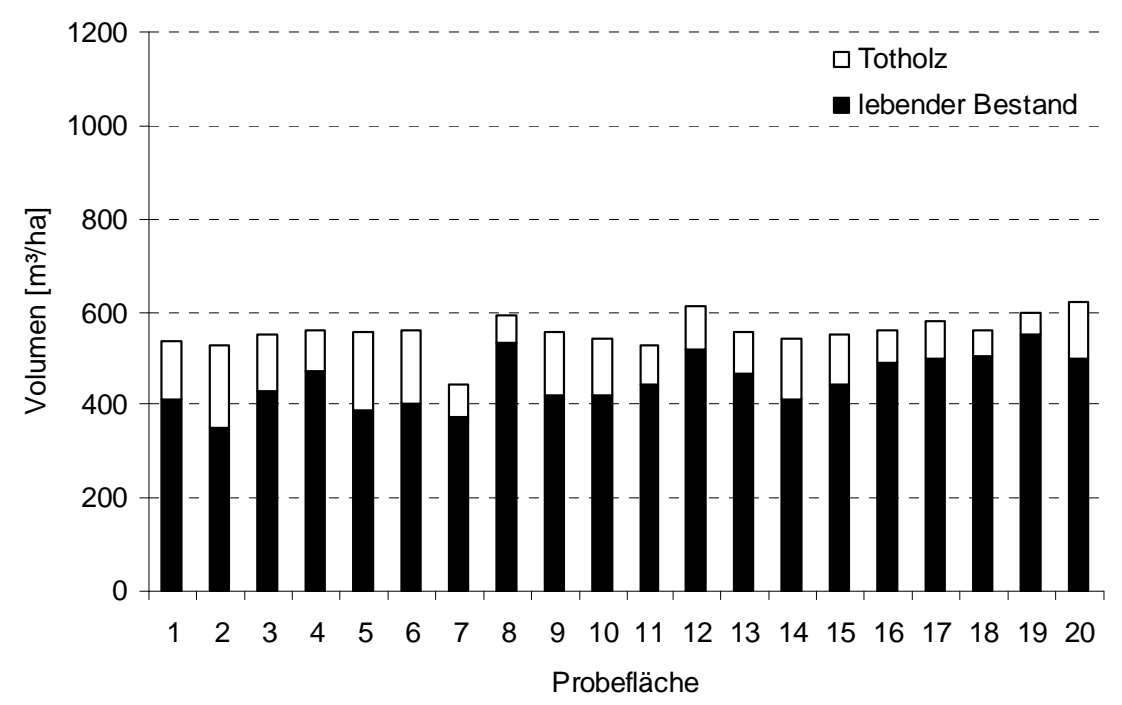

Abb. 3. Derbholzvorrat (lebend und tot) auf 20 Probeflächen in Kyjov (Die Strukturvielfalt im Bestandesaufbau nimmt nach rechts zu.)

\subsubsection{Totholzvorrat}

Der Totholzvorrat variierte in Havešová von 33 bis $285 \mathrm{~m}^{3} \mathrm{ha}^{-1}$ (Abb. 2) und lag im Durchschnitt bei $128 \mathrm{~m}^{3} \mathrm{ha}^{-1}$ (Standardfehler: $\pm 15 \mathrm{~m}^{3} \mathrm{ha}^{-1}$ ). Davon nahm das liegende Totholz $101 \mathrm{~m}^{3} \mathrm{ha}^{-1}$ und das stehende Totholz $27 \mathrm{~m}^{3} \mathrm{ha}^{-1}$ ein. Das Verhältnis zwischen dem Holzvorrat lebender Bäume und dem Totholzvorrat betrug durchschnittlich 5,5:1. Der mittlere Totholzanteil am Gesamtholzvolumen betrug $16 \%$ (Standardfehler: 1,9\%) und schwankte auf den einzelnen Flächen zwischen 4 und $37 \%$. Die kleinflächige Verteilung von Totholz

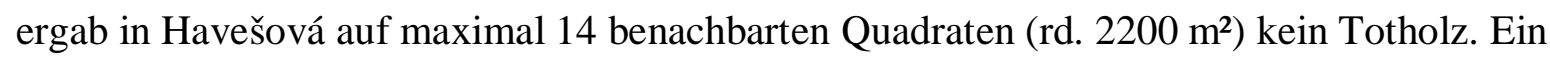
besonders hoher Totholzvorrat von $304 \mathrm{~m}^{3} \mathrm{ha}^{-1}$ wurde in einem Fall auf 9 benachbarten Quadraten (rd. $1400 \mathrm{~m}^{2}$ ) erreicht.

In Kyjov war der Totholzvorrat im Unterschied zum Vorrat des lebenden Bestandes nicht wesentlich geringer als in Havešová. Er variierte zwischen 45 und $176 \mathrm{~m}^{3} \mathrm{ha}^{-1}$ (Abb. 3) und ergab im Durchschnitt $110 \mathrm{~m}^{3} \mathrm{ha}^{-1}$ (Standardfehler: $\pm 8 \mathrm{~m}^{3} \mathrm{ha}^{-1}$ ). Davon nahm das liegende Totholz $77 \mathrm{~m}^{3} \mathrm{ha}^{-1}$ und das stehende Totholz $33 \mathrm{~m}^{3} \mathrm{ha}^{-1}$ ein. Das Verhältnis zwischen dem Vorrat lebender Bäume und dem Totholzvorrat betrug 4,1:1. Der mittlere Totholzanteil von $19 \%$ (Standardfehler: 1,6 \%) schwankte zwischen 8 und $33 \%$. Frei von starkem Totholz waren maximal 15 benachbarte Quadrate (rd. $2300 \mathrm{~m}^{2}$ ). In einem Fall ergab sich auf $1400 \mathrm{~m}^{2}$ ein besonders hoher Totholzvorrat von $278 \mathrm{~m}^{3} \mathrm{ha}^{-1}$.

Regressionsanalysen zeigten in beiden Reservaten einen signifikanten Zusammenhang zwischen dem Vorrat des lebenden Bestandes und dem Totholzvorrat auf den Stichprobenflächen (62,5 m x 62,5 m), wobei der Vorrat des lebenden Bestandes 
Veränderungen des Totholzvorrats mit Hilfe eines linearen Modells zu rd. 50 \% erklärte. Je größer der Vorrat des lebenden Bestandes war, umso geringer war das Totholzvolumen. Auf den 12,5 m x 12,5 m-Quadraten ergab sich auch ein signifikanter Zusammenhang, der jedoch von geringer Relevanz war $\left(\mathrm{R}^{2}<0,05\right)$.

In Havešová waren $53 \%$ der toten Bäume entwurzelt und $47 \%$ abgebrochen, während in Kyjov 22,5 \% der Bäume entwurzelt, $76 \%$ abgebrochen und 1,5\% stehend abgestorben waren. Windwürfe von gleichzeitig mehreren Bäumen traten mit ähnlicher Frequenz und Größe auf wie abgebrochene Bäume.

Regressionsanalysen zeigten für Kyjov auf den Stichprobenflächen (62,5 m x 62,5 m) signifikante Zusammenhänge zwischen dem strukturellen Komplexitätsindex und dem Vorrat des lebenden Bestandes sowie zwischen dem Komplexitätsindex und dem Totholzvorrat. So erklärte ein ansteigender Komplexitätsindex zu 46 \% die Zunahme des Holzvorrats und zu 36 $\%$ die Abnahme des Totholzvorrats (lineare Modelle). In Havešová ergaben sich dagegen keine signifikanten Zusammenhänge.

Für Regressionen zwischen Holzvorrat und Diffuse-Site-Factor konnte in beiden Reservaten kein signifikanter Zusammenhang festgestellt werden.

\subsubsection{Bestandesdichte}

Die Grundfläche variierte in Havešová zwischen 25 und $45 \mathrm{~m}^{2} \mathrm{ha}^{-1}$, der Durchschnitt betrug $35 \mathrm{~m}^{2} \mathrm{ha}^{-1}$ (Abb. 4). In Kyjov lagen die Werte zwischen 25 und $40 \mathrm{~m}^{2}$ ha $^{-1}$ (Abb. 5). Der Durchschnitt betrug $32 \mathrm{~m}^{2} \mathrm{ha}^{-1}$.

Ein Zusammenhang zwischen der Grundfläche und dem strukturellen Komplexitätsindex von Zenner u. Hibbs (2000) stellte sich in Havešová nicht heraus. In Kyjov wurden auf heterogener aufgebauten Flächen etwas größere Grundflächen festgestellt (siehe Abb. 5). Der Index schwankte auf den Probeflächen in Havešová von 3,06 bis 4,96 und in Kyjov von 1,98 bis 2,83 . 


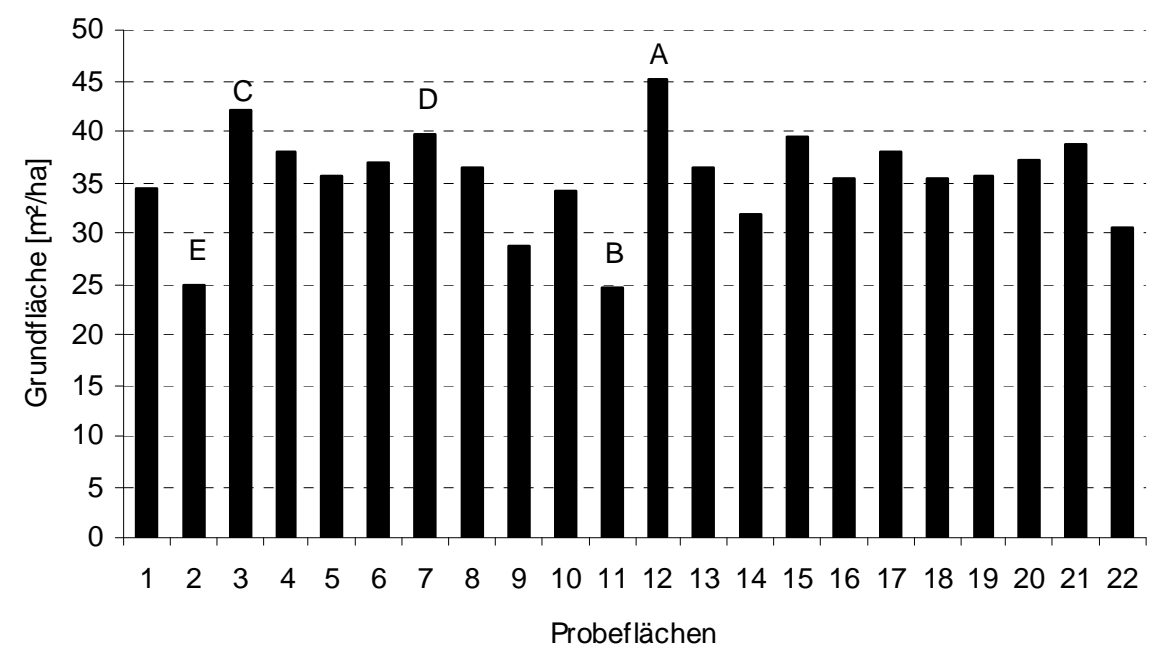

Abb. 4. Grundfläche aller Bäume mit $\geq 7 \mathrm{~cm}$ BHD auf 22 Probeflächen in Havešová (Die Strukturvielfalt im Bestandesaufbau nimmt nach rechts zu. Die Probeflächen A-E wurden genetisch untersucht, siehe Abschnitt 2.3.5)

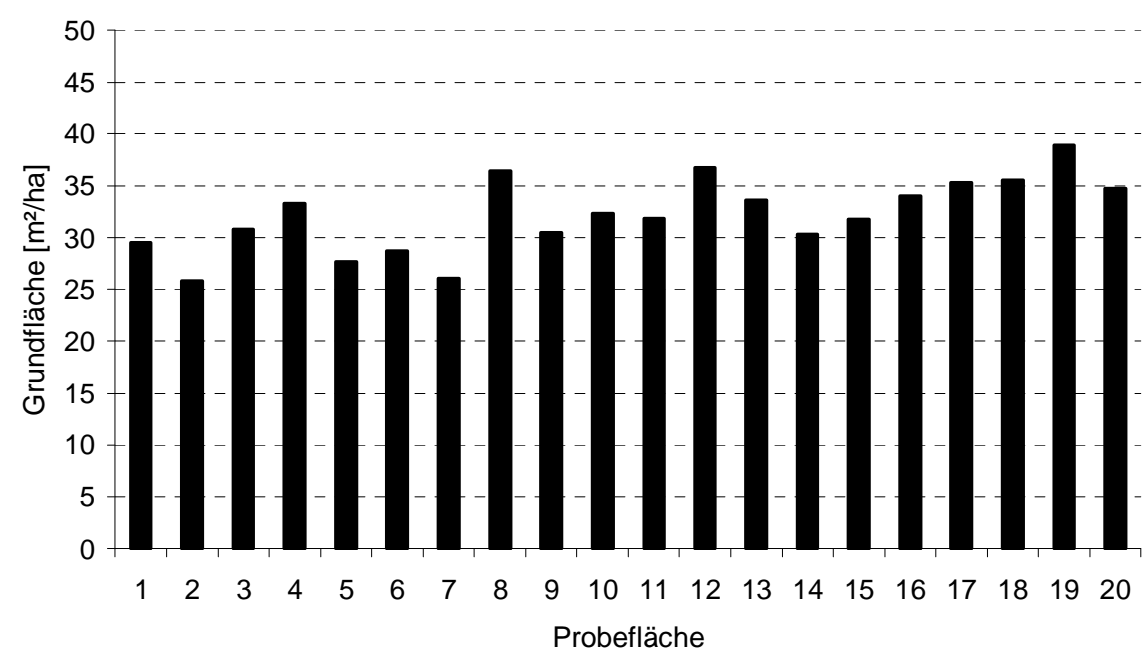

Abb. 5. Grundfläche aller Bäume mit $\geq 7 \mathrm{~cm}$ BHD auf 20 Probeflächen in Kyjov (Die Strukturvielfalt im Bestandesaufbau nimmt nach rechts zu.)

Die Anzahl der Bäume betrug auf den einzelnen Probeflächen in Havešová zwischen 200 und 400 Stück pro ha (Abb. 6) und in Kyjov zwischen 250 und 400 Stück pro ha (Abb. 7).

Durchschnittlich standen in Havešová 290 Bäume und in Kyjov 320 Bäume auf einem ha. 


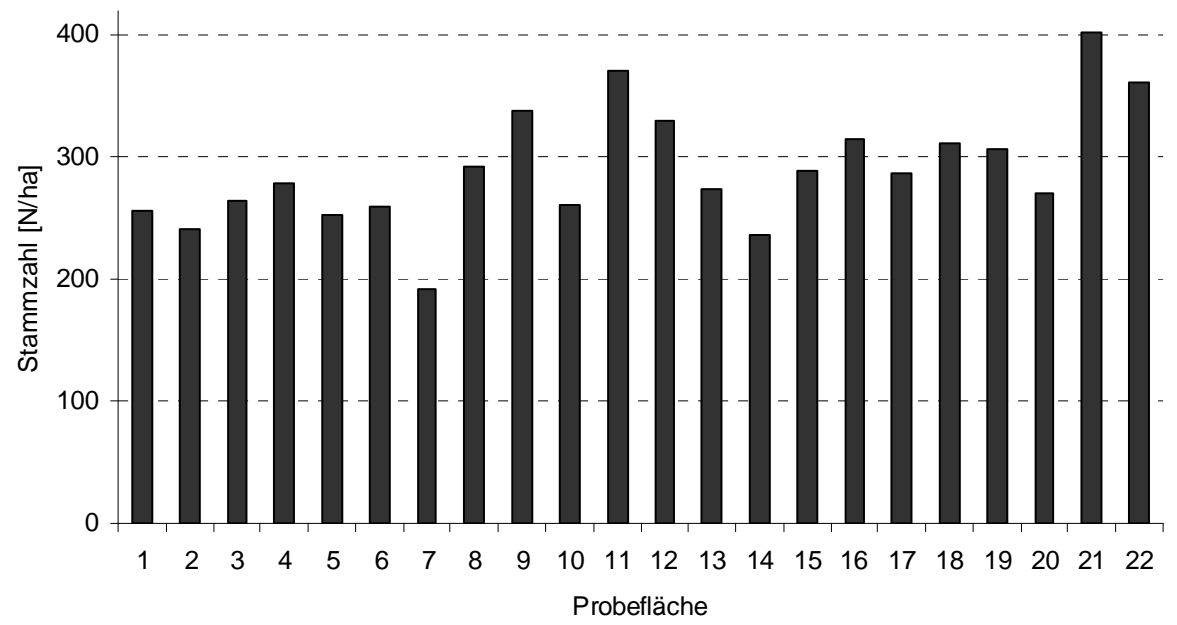

Abb. 6. Stammzahl aller Bäume mit $\geq 7 \mathrm{~cm}$ BHD auf 22 Probeflächen in Havešová (Die Strukturvielfalt im Bestandesaufbau nimmt nach rechts zu.)

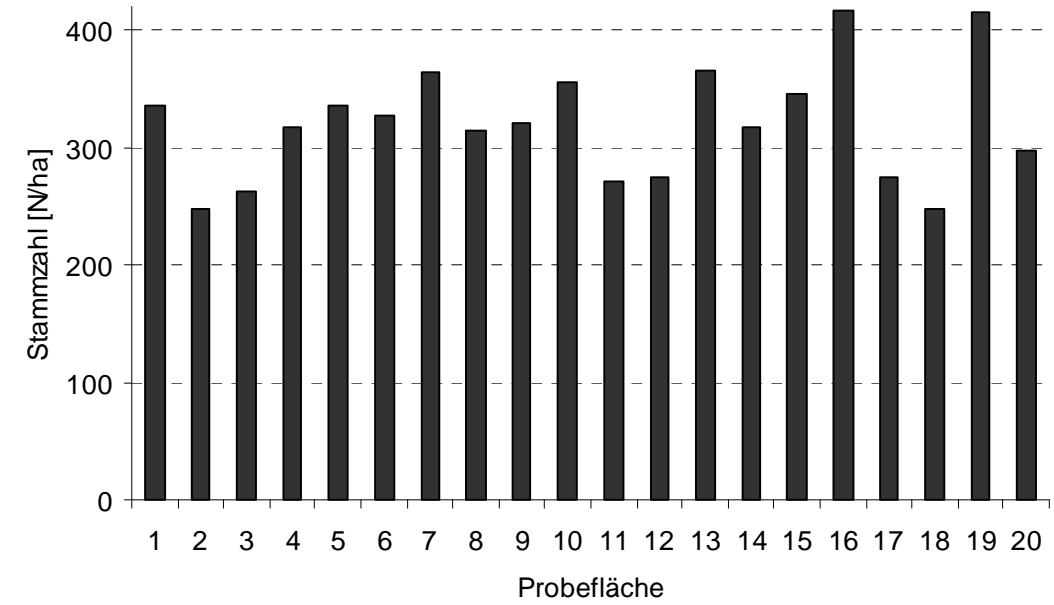

Abb. 7. Stammzahl aller Bäume mit $\geq 7 \mathrm{~cm}$ BHD auf 20 Probeflächen in Kyjov (Die Strukturvielfalt im Bestandesaufbau nimmt nach rechts zu.) 


\subsubsection{Durchmesserverteilung}

In Abb. 8 und 9 ist die Durchmesserverteilung dargestellt. Dafür wurden die

Durchmesserverteilungen der einzelnen Stichprobenflächen zusammengefasst. In beiden

Reservaten nahm die Stammzahl mit zunehmendem Durchmesser ständig ab. In Kyjov traten jedoch Bäume mit 7 bis $11 \mathrm{~cm}$ BHD seltener auf, während Bäume mit 12-20 cm BHD häufiger vorkamen.

Der größte gemessene BHD betrug in Havešová 127 cm, der mittlere BHD 29,3 cm und der Durchmesser des Grundflächenmittelstamms $\left(\mathrm{d}_{\mathrm{g}}\right) 38,8 \mathrm{~cm}$. In Kyjov betrug der maximale BHD $121 \mathrm{~cm}$, der mittlere BHD 27,6 cm und der $\mathrm{d}_{\mathrm{g}} 35,4 \mathrm{~cm}$.

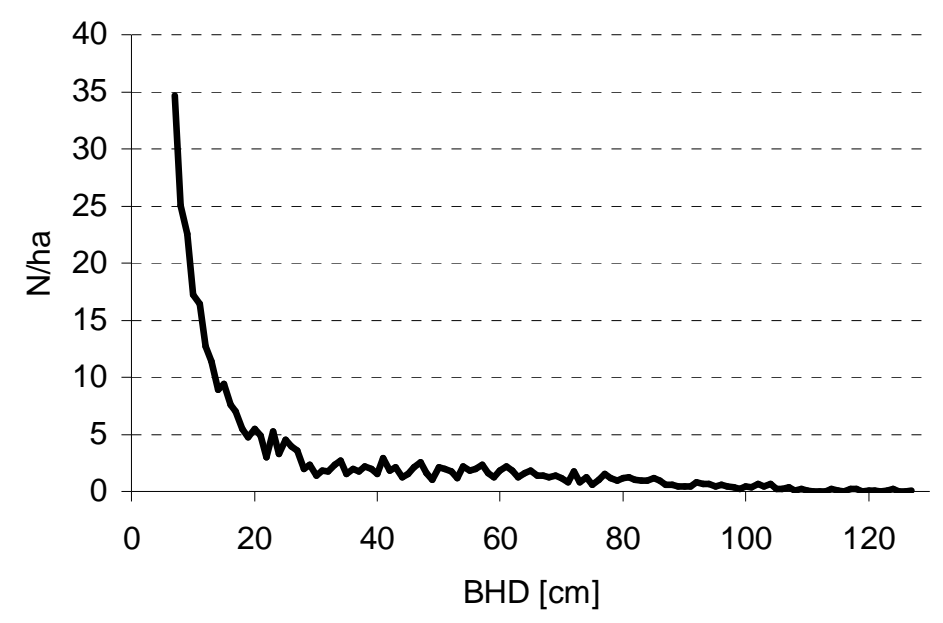

Abb. 8. Durchmesserhäufigkeitsverteilung in Havešová

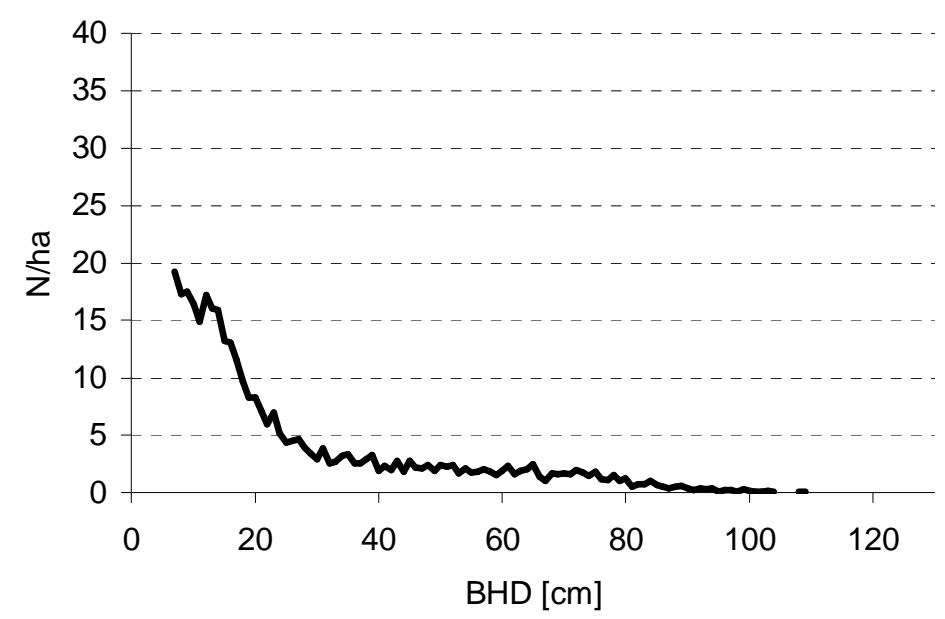

Abb. 9. Durchmesserhäufigkeitsverteilung in Kyjov 


\subsubsection{Baumhöhen und Kronendimension}

Die höchsten Bäume in Havešová erreichten knapp 50 m (Abb. 10). Eine extrem hohe Buche war 56 m hoch. Der Kronenansatz der Bäume der Oberschicht (ab $30 \mathrm{~cm}$ BHD) lag in $18 \mathrm{~m}$ Höhe. Die höchsten Bäume in Kyjov waren 35 m hoch (Abb. 11). Der Kronenansatz der Bäume der Oberschicht (ab 20 cm BHD) lag in 11 m Höhe.

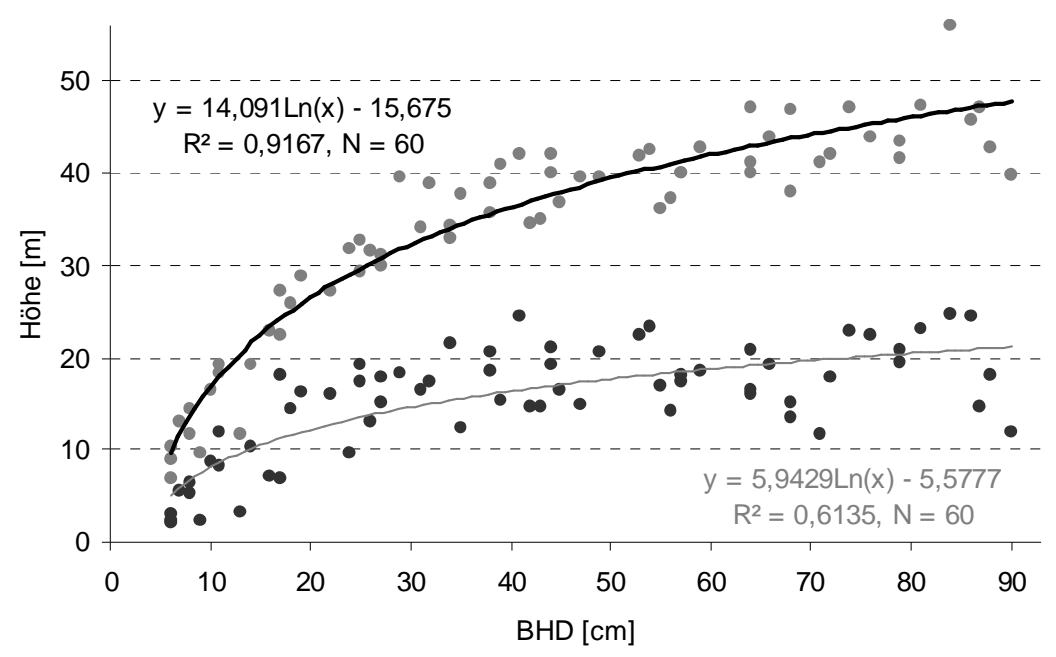

Abb. 10. Bestandeshöhenkurve und Kronenansatzhöhen in Havešová

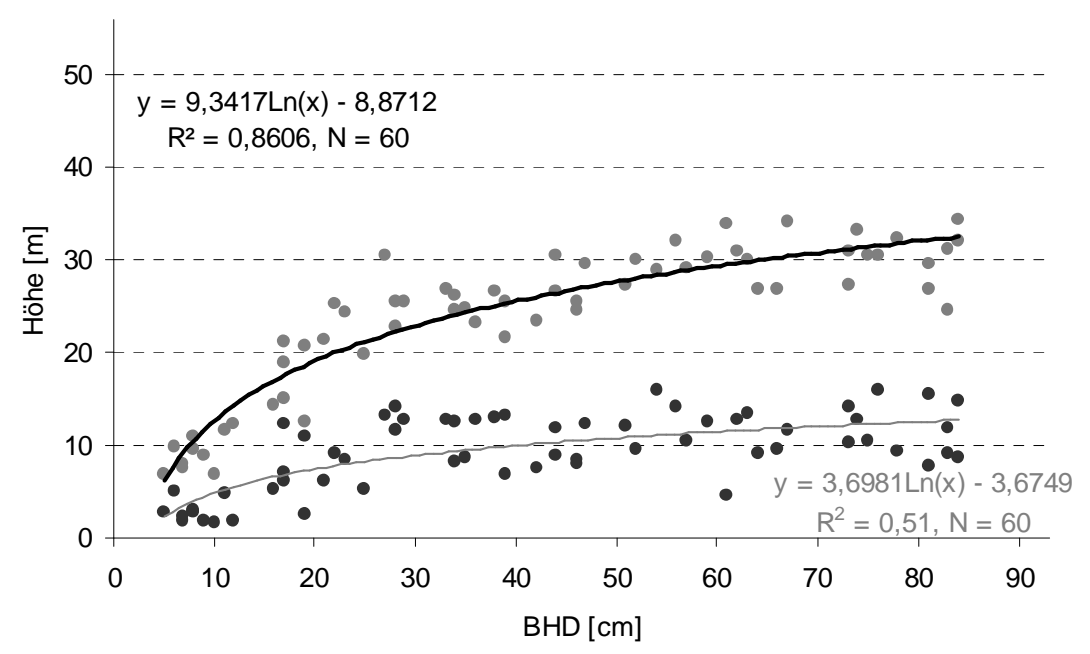

Abb. 11. Bestandeshöhenkurve und Kronenansatzhöhen in Kyjov

Bäume der Oberschicht (definiert nach Leibundgut 1993) besaßen in Havešová eine durchschnittliche Kronenlänge von $18 \mathrm{~m}$ und in Kyjov von $17 \mathrm{~m}$. In Havešová betrug der Schlankheitsgrad (= Höhe/BHD) 139 für Bäume der Unter- u. Mittelschicht und 67 für Bäume der Oberschicht. In Kyjov besaßen die Bäume der Unter- u. Mittelschicht einen Schlankheitsgrad von 103 und die Bäume der oberen Bestandesschicht einen Wert von 60. 
Die Abb. 12 und 13 zeigen einen engen und in beiden Beständen fast gleichartigen Zusammenhang zwischen dem BHD und dem mittleren Kronenradius der Bäume. In beiden Reservaten besaßen $80 \mathrm{~cm}$ starke Bäume einen mittleren Kronenradius von 8 m, was (bei angenommener Kreisform) einer Kronenschirmfläche von $200 \mathrm{~m}^{2}$ entspricht. Bäume mit 40 cm BHD besaßen im Durchschnitt eine Krone mit 5 m Radius, was einer Fläche von 80 m² $^{2}$ entspricht.

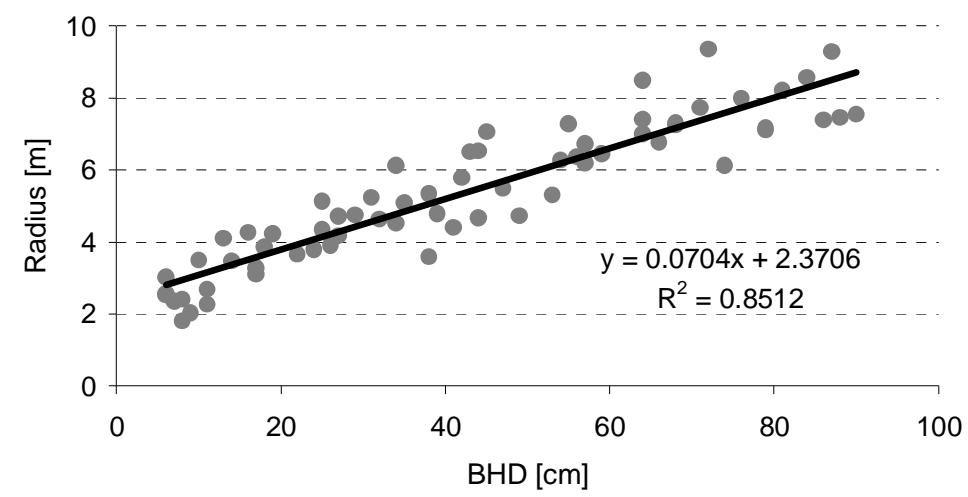

Abb. 12. Zusammenhang zwischen Kronenradius und BHD in Havešová

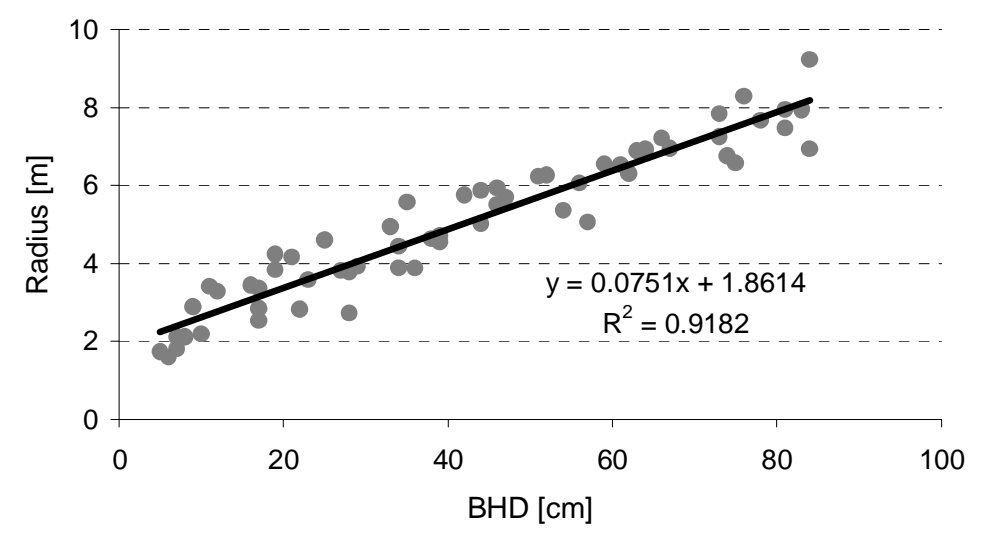

Abb. 13. Zusammenhang zwischen Kronenradius und BHD in Kyjov

\subsection{DISKUSSION}

\subsubsection{Methodik}

Die Aufnahme erfolgte in den Reservaten auf 20 bzw. 22 systematisch verteilten

Probeflächen. Im Unterschied zur Aufnahme einer zusammenhängenden Fläche (Kernfläche) konnten so statistische Streuungsparameter ermittelt werden. Dadurch ließen sich unter anderem Volumenangaben von Korpel' (1995) in den beiden Reservaten überprüfen, die auf drei bzw. vier 0,5 ha großen Flächen in unterschiedlichen Waldentwicklungsstadien erhoben 
wurden. Außerdem konnte durch systematische Stichproben das Problem umgangen werden, wie weit eine solche Kernfläche repräsentativ für die Verhältnisse im gesamten Reservat ist.

Die Zerschneidung der Aufnahmefläche schränkte die Auswertung der kleinflächigen

Verteilung der Holzvorräte etwas ein. In einem etwa 300 km entfernten Buchen-

Urwaldreservat in der Ukraine wurde dagegen eine 10 ha große Fläche aufgenommen

(Commarmot et al. 2005), die eine Analyse ohne große Randeffekte erlaubt.

\subsubsection{Boden, Krautvegetation und Verjüngung}

Die Böden der slowakischen Reservate unterscheiden sich im Ausgangssubstrat mit Flysch und Andesit von den anschließend diskutierten albanischen Buchenurwäldern mit Gabbro und Serpentin und deutschen Beständen mit Muschelkalk (Bleicherode, Göttinger Wald) oder Buntsandstein (Limker Strang, Solling). Erstere sind durch höhere Kalium- und

Natriumvorräte und deutlich geringere Magnesiumvorräte gekennzeichnet. Für den Vergleich mit deutschen Wirtschaftswäldern werden die Bewertungsmaßstäbe der

Bodenzustandserhebung (Wolff u. Riek 1997) herangezogen. Dafür war eine Umrechnung der Daten aus Tabelle 4 und 5 in die Einheit $\mathrm{kg} \mathrm{ha}^{-1}$ bis $30 \mathrm{~cm}$ Mineralbodentiefe notwendig. Die Bodendichte betrug in Havešová 1,3 $\mathrm{g} \mathrm{cm}^{-3}$ und in Kyjov $1,2 \mathrm{~g} \mathrm{~cm}^{-3}$ (Pichler et al. 2005).

Tab. 12. Elementvorräte $[\mathrm{kg} / \mathrm{ha}]$ in 0-30 cm Tiefe des Mineralbodens mit einer Bewertung entsprechend Wolff u. Riek (1997). Wenn keine Bewertung vorgenommen wurde, sind Median und Extremwerte aus allen Proben der Bodenzustandserhebung angegeben.

\begin{tabular}{|c|c|c|c|c|}
\hline Element & $\begin{array}{c}\text { Havešová } \\
{[\mathrm{kg} / \mathrm{ha}]}\end{array}$ & Bewertung & $\begin{array}{c}\text { Kyjov } \\
{[\mathrm{kg} / \mathrm{ha}]}\end{array}$ & Bewertung \\
\hline C & 65416 & gering & 212184 & hoch \\
\hline $\mathrm{N}$ & 7579 & mittel & 19932 & hoch \\
\hline $\mathrm{P}$ (pflanzenverfügbar) & 13 & hoch* ${ }^{*}$ - & 14 & hoch* \\
\hline $\mathrm{Ca}$ & 3750 & mittel/hoch & 2306 & $\mathrm{mittel} / \mathrm{hoch}$ \\
\hline K & 974 & mittel/hoch & 1070 & $\mathrm{mittel} / \mathrm{hoch}$ \\
\hline $\mathrm{Mg}$ & 313 & mittel & 180 & mäßig \\
\hline $\mathrm{Na}$ & 96 & k. A. & 75 & k. A. \\
\hline $\mathrm{Fe}$ & 7 & Min. 1, Median 402, Max. 13162 & 3 & \\
\hline $\mathrm{Mn}$ & 175 & Min. 1, Median 118, Max. 3534 & 56 & \\
\hline $\mathrm{Al}$ & 1996 & Min. 10, Median 1420, Max. 29160 & 2794 & \\
\hline $\mathrm{H}$ & 2 & Min. 0, Median 7, Max. 106 & 2 & \\
\hline $\mathrm{C} / \mathrm{N}$ & $9,1: 1$ & eng* ${ }^{*}-10$ & $10,5: 1$ & eng* \\
\hline AKe & $5,5 \mathrm{cmol} / \mathrm{kg}$ & mittel $^{\star *}$ & $5,6 \mathrm{cmol} / \mathrm{kg}$ & mittel $^{\star *}$ \\
\hline Basensättigung & $61 \%$ & mäßig hoch & $47 \%$ & mittel \\
\hline
\end{tabular}

* Bewertung des P-Gehaltes und C/N-Verhältnisses im Vergleich zu Buchenwäldern von Schmidt (1970)

** Bewertung der AKe im Vergleich zu Buchenwäldern von Tabaku (1999) 
Der Kohlenstoffanteil im Mineralboden in Havešová entspricht dem Rahmen, der in bewirtschafteten Buchenbeständen beobachtet wurde und zwischen 57 und $122 \mathrm{t} / \mathrm{ha}$ liegt (Mund 2004, Schöning 2003). In Kyjov werden diese Werte erheblich überschritten. Die einzelnen Profile zeigen jedoch einen typischen, nach unten abnehmenden Gradienten des Kohlenstoffgehalts ohne Ausreißer. Ähnlich hohe Werte stellte Tabaku (1999) im Urwaldreservat Rajca in Albanien +fest: 9,0 \% in 0-10 cm Tiefe und 3,2\% in 20-30 cm Tiefe. Die hohen Anteile in Kyjov und Rajca sprechen für eine Kohlenstoffakkumulation im Boden von Urwäldern, der Anteil in Havešová demonstriert jedoch das Gegenteil. Möglicherweise ist die Akkumulation gerade in Urwäldern besonders abhängig vom Bodentyp und anderen Merkmalen, deren großer Einfluss auf die C-Speicherung im Boden von Wirtschaftswäldern bereits festgestellt und für eine große Variation verantwortlich gemacht wurde (Brumme et al. 2006). Zuverlässigere Aussagen setzen allerdings weitere Untersuchungen voraus. Der Stickstoffanteil ist in Kyjov zwei- bis dreimal höher als in den anderen Reservaten. Im Vergleich zu albanischen Buchenurwäldern (Tabaku 1999) ist das C/N-Verhältnis in den slowakischen Reservaten nur halb so groß. Schmidt (1970) stellte im humosen Oberboden von 20 Braunerde-Buchenwäldern ein C/N-Verhältnis von 14 fest. Das besonders enge Verhältnis von 10 der slowakischen Reservate weist auf eine schnellere Zersetzung des Kohlenstoffs hin. Wie beim Kohlenstoff lassen die hohen N-Anteile in Kyjov eine Akkumulation unter den ungestörten Bodenverhältnissen eines Urwaldes vermuten. Die niedrigen Anteile in Havešová widersprechen wiederum dieser Vermutung.

Die Magnesium- und Kalziumversorgung war in Kyjov etwas schlechter als in Havešová. Es wurde aber in keinem Reservat ein Mangel an einem Nährelement festgestellt. Die Basensättigung lag im mittleren Bereich. Der geringe Anteil austauschbarer H- und Fe-Ionen an der effektiven Austauschkapazität (rd. $1 \%$ ) lässt auf eine hohe Elastizität gegenüber Säurebelastung schließen.

Beide Braunerden sind mit ihrer guten Nährstoffversorgung zwischen den KalkBuchenwäldern und bodensauren Buchenwäldern einzuordnen. Geht man entsprechend der Korngrößenfraktionen (Tabelle 2) von tonhaltigen Lehmböden, einer mittleren Lagerungsdichte (Pichler et al. 2005) und jährlichen Niederschlägen um $750 \mathrm{~mm}$ in beiden Reservaten aus, kann man nach Hoffmann (1996) unter Berücksichtigung der Hanglage von sehr frischen Standorten ausgehen, die keine Probleme mit der Wasserversorgung haben. Zusätzlich kann man in den ungestörten Beständen von einer intensiveren Durchwurzelung ausgehen, welche eine gute Wasserversorgung gewährleistet. 
Zusätzlich zu unserer Arbeit untersuchte Jaloviar (2005) in Havešová an den gleichen

Bodenprobepunkten die Feinwurzelmasse (Oberboden bis $40 \mathrm{~cm}$ ). Die Proben wurden im Juli 2004 entnommen. Er ermittelte 4,5 $\mathrm{t} \mathrm{ha}^{-1}$ lebende Feinwurzeln und $2 \mathrm{t} \mathrm{ha}^{-1}$ abgestorbene Feinwurzeln. Diese Werte lagen auch im Göttinger Wald vor (Hertel 1999), allerdings mit einer kleineren Masse abgestorbener Feinwurzeln.

Von der Artenzusammensetzung der Krautvegetation ähneln die slowakischen Bestände den

Waldmeister-Buchenwäldern in Deutschland. Auf einer Aufnahmefläche in Havešová ist eine Tendenz zum Carici pilosae-Fagetum erkennbar. Die vorgefundenen Arten Dentaria bulbifera, Paris quadrifolia und Polygonatum verticillatum werden von Müller (1989) und Dierschke (1989) als Trennarten für montane Höhenformen des Galio- und HordelymoFagetum angesehen.

In Tabelle 13 wurden Durchschnittswerte aus den ökologischen Ansprüchen der Arten bezüglich Licht, Temperatur, Kontinentalität, Wasserhaushalt, Bodenreaktion und Stickstoffversorgung berechnet.

Tab. 13. Durchschnittliche Zeigerwerte der vorgefundenen Pflanzenarten nach Ellenberg (2001) und Zolyomi (1966)

\begin{tabular}{lcccccccc}
\hline & $\mathrm{L}$ & $\mathrm{T}$ & $\mathrm{K}$ & $\mathrm{WHS}$ & $\mathrm{F}$ & $\mathrm{HUF}$ & $\mathrm{R}$ & $\mathrm{N}$ \\
\hline \hline Havešová & 3,2 & 4,9 & 3,9 & II & 5,5 & 3 & 5,2 & 6,0 \\
Kyjov & 4,1 & 4,3 & 3,8 & II-III & 5,6 & 3,2 & 5,4 & 5,8 \\
\hline
\end{tabular}

Schattenpflanzen dominieren in Havešová. In Kyjov kamen Halbschattenpflanzen hinzu, die aber meist auf feuchte Stellen begrenzt blieben. In Havešová zeigen die Arten mäßige Wärme und ein subozeanisches Klima an, in Kyjov kommen zusätzlich Kühlezeiger vor. In beiden Reservaten handelt es sich um typische Arten für einen frischen Standort, die bessere ModerHumusformen anzeigen. Die Böden sind zwischen stickstoffreich und mäßig stickstoffreich einzustufen. Reaktionszahlen um 5 weisen auf einen mäßig sauren pH-Bereich hin, was mit den Messungen übereinstimmt (Tab. 4).

Die Verjüngung war in beiden Reservaten zahlreich vorhanden. In Kyjov produzierte der Bestand ein Jahr vor der Aufnahme eine Buchenmast, welche einen besonders hohen Buchenanteil in der kleinsten Höhenklasse verursachte. Der relative Anteil der Pflanzen von 0,5 bis $3 \mathrm{~m}$ Höhe war im Vergleich zu albanischen Urwäldern kleiner (Tabaku 1999). Die Verjüngung war in allen Urwaldreservaten nahezu flächig vorhanden, wie der hohe Anteil besetzter Probekreise von rd. 90 \% zeigt. 
Als Grund für die schnelle Abnahme der Zahl der Nebenbaumarten bis 1 m Höhe kann Wildeinfluss ausgeschlossen werden, da nur eine sehr geringe Verbissbelastung festgestellt wurde. Das geringe Strahlungsangebot kann dagegen eine große Rolle spielen. In den größeren Höhenklassen ist das Auftreten der Nebenbaumarten zu gering, um Aussagen zur Konkurrenz mit Buchen treffen oder Zusammenhänge mit dem Lichtangebot erkennen zu können.

Die Lichtverhältnisse am Boden unterschieden sich von denen in albanischen BuchenUrwäldern. Dort schwankten die Werte von 4,3 bis 6,7 \% (Tabaku 1999). Diaci und Thormann (2002) ermittelten in einem slowenischen Buchennaturwald mit vereinzelten Nadel- und Edellaubbäumen einen Diffuse-Site-Factor von durchschnittlich 4,7 \%. Das Lichtangebot in Kyjov war daher mit 9,4 \% im Vergleich zu anderen Buchenurwäldern relativ hoch. Es entsprach dem Durchschnittswert, der von Tabaku (1999) im Buchenplenterwald Bleicherode in Thüringen festgestellt wurde. Der Diffuse-Site-Factor in Havešová ähnelte mit 2,5\% dem Durchschnittswert von $2 \%$ im Naturwaldreservat Limker Strang, das sich in der mittleren und späten Optimalphase befand (Tabaku 1999). Im Vergleich mit anderen Urwaldreservaten waren die Lichtverhältnisse in Havešová niedrig.

Das geringe Lichtangebot in Havešová lässt sich zum Teil durch sehr hohe Bäume erklären, die den Einstrahlungswinkel in Lücken verkleinern. Das erhöhte Lichtangebot in Kyjov im Vergleich zu albanischen Urwäldern lässt auf stärkere Störungen schließen. Eine Ausscheidung von Waldentwicklungsphasen durch Drößler u. Meyer (2006) ergab dementsprechend einen höheren Anteil der Phasen mit geringer Überschirmung in Kyjov.

\subsubsection{Die Vergleichbarkeit mit Buchenbeständen in Deutschland}

Um aus Urwäldern Rückschlüsse für bewirtschaftete Wälder (Vorratshaltung, Störungsregime etc.) ziehen zu können, muss man klimatische, standörtliche und pflanzenspezifische Unterschiede einschätzen können. Eine vollständige Übereinstimmung mit den Wuchsbedingungen in Deutschland ist sicher nicht gegeben, die herangezogenen ökologischen Kriterien lassen aber insgesamt auf eine gute Vergleichbarkeit schließen: Die Buche besitzt eine weite Standortamplitude, wie auch das Ökogramm aus Leuschner (1997) zeigt. pH-Werte im Boden können 3 bis über 7 betragen und spielen keine entscheidende Rolle (Leuschner 1998). Die Humusform (Rohhumus bis Mull) und das Nährstoffangebot begrenzen die Buche in ihrem Vorkommen ebenfalls nicht (Leuschner et al. 1993). Ihr Wuchsoptimum findet sie auf ,tiefgründigen, nachhaltig frischen, basenreichen, gut durchlüfteten und drainierten Braunerden“ (Mayer 1984, zit. aus Czajkowski et al. 2006). 
Anders als der Standort besitzt das Klima einen großen Einfluss auf das Vorkommen der Buche: Lange, strenge Winter und Sommertrockenheit begrenzen ihre Verbreitung im Osten (Tarasiuk 1999). De Candolle (1855) und Willkomm (1887, beide zit. aus Czajkowski et al. 2006) heben die Bedeutung der durchschnittlichen Wintertemperatur über $-6{ }^{\circ} \mathrm{C}$ und der Niederschlagsmenge in der Vegetationsperiode hervor. Ellenberg (1996) leitet dagegen aus dem Verhältnis der Durchschnittstemperatur im Juli und dem Jahresniederschlag einen Weiser ab. Niederschläge über $450 \mathrm{~mm}$ in der Vegetationsperiode stellen keinen Limitierungsfaktor dar. In Uzhgorod (25 km Luftlinie von den Reservaten entfernt, auf $118 \mathrm{~m}$ ü. NN) beträgt die durchschnittliche Temperatur im Januar $-3^{\circ} \mathrm{C}$ und im Juli $20^{\circ} \mathrm{C}$ (Mühr 2002). Im Frühjahr sind Spätfröste die größte Gefahr für Keimlinge und Blüten (Röhrig et al. 2006). Spätfrostschäden konnten aber in beiden Reservaten nicht entdeckt werden. Der frühere Blattaustrieb der Buchen aus Havešová im Vergleich zu Buchen aus dem Solling kann sogar als eine Anpassung an eine geringe Spätfrostgefahr interpretiert werden. In einem Vergleich der Frostresistenz von Buchen entlang eines Nord-Süd-Gradienten beschreiben Višnjic u. Dohrenbusch (2002) eine rumänische Herkunft, die im Vergleich zu einer Herkunft aus dem Solling 11 Tage früher austrieb.

Insgesamt zeigten die Untersuchungsbestände eine sehr gute Höhenwuchsleistung und eine große Konkurrenzkraft gegenüber anderen Baumarten. Deshalb kann man davon ausgehen, dass das im Vergleich zu Deutschland kontinentaler geprägte Klima keine großen Einschränkungen im Wachstum und im Bestandesaufbau im Vergleich zu Buchenwäldern in Deutschland hervorruft. Diese Annahme wird auch dadurch gestützt, dass Buchenurwälder auf noch unterschiedlicheren Standorten, z.B. in Albanien (Tabaku 1999, Tabaku u. Meyer 1999) oder Slowenien (Zeibig et al. 2005), sehr ähnliche Bestockungsverhältnisse und Lückenmuster aufweisen.

Die genetischen Untersuchungen zeigen ebenfalls, dass die slowakischen Buchenbestände in einem Rahmen liegen, der die Vergleichbarkeit mit deutschen Buchenbeständen erlaubt (Konnert et al. 2000). Der deutlich geringere Stichprobenumfang in Havešová gegenüber Beständen in Deutschland kann zu größeren Unterschieden innerhalb des Bestandes beitragen.

\subsubsection{Holzvorrat und Bestandesdichte}

Von den drei von Tabaku (1999) untersuchten albanischen Buchen-Urwaldreservaten entsprach der am geringsten bevorratete Bestand Mirdita mit $559 \mathrm{~m}^{3} \mathrm{ha}^{-1}$ am ehesten dem Bestand in Kyjov. Die anderen beiden Reservate wiesen etwa $800 \mathrm{~m}^{3} \mathrm{ha}^{-1}$ auf. Die maximalen Baumhöhen in Mirdita entsprachen denen in Kyjov. Die höchsten Bäume in den anderen 
beiden albanischen Reservaten waren etwa 40 m hoch (Tabaku 1999). Korpel' (1995) ermittelte in Kyjov auf vier 0,5 ha großen Flächen 400 bis $600 \mathrm{~m}^{3} \mathrm{ha}^{-1}$ Holzvorrat und in Havešová auf drei 0,5 ha großen Flächen zwischen 600 und $800 \mathrm{~m}^{3} \mathrm{ha}^{-1}$. In diesem Rahmen liegen auch die Vorräte in unserer Untersuchung. Dazu passen die ebenfalls von Korpel' (1995) festgestellten Durchschnittswerte in zwei benachbarten Buchen-Urwaldreservaten Rožok und Stužica von $740 \mathrm{~m}^{3}$ bzw. $570 \mathrm{~m}^{3}$ je ha. Commarmot et al. (2005) ermittelten für den Urwald Uholka in der Ukraine einen Vorrat von durchschnittlich $770 \mathrm{~m}^{3} \mathrm{ha}^{-1}$, der auf 0,25 ha großen Unterflächen von 421 bis $1042 \mathrm{~m}^{3} \mathrm{ha}^{-1}$ variierte. Chernyavskyy (2005) stellte in diesen Urwäldern Vorräte je nach Entwicklungsphase von 435 und $540 \mathrm{~m}^{3} \mathrm{ha}^{-1}$ fest. Tsuryk (1980) gibt 588 und $678 \mathrm{~m}^{3} \mathrm{ha}^{-1}$ an. Aufgrund all dieser Untersuchungen kann man je nach Wüchsigkeit im Buchenurwald von durchschnittlich $450 \mathrm{~m}^{3} \mathrm{ha}^{-1}$ (30 m Oberhöhe) bis $800 \mathrm{~m}^{3}$ $\mathrm{ha}^{-1}$ (etwa $40 \mathrm{~m}$ Oberhöhe) ausgehen.

Tab. 14. Holzvorrat, Totholzvorrat und Grundfläche [Angaben je ha] von BuchenUrwaldreservaten, die bis jetzt publiziert wurden.

\begin{tabular}{|c|c|c|c|c|c|c|}
\hline Reservat & Land & Quelle & $\begin{array}{c}\text { Größe der } \\
\text { Aufnahmefläche }\end{array}$ & Holzvorrat & Totholzvorrat & Grundfläche \\
\hline Havešová & Slowakei & Text & $22 \times 0,39$ ha & $716 \mathrm{~m}^{3}$ & $128 \mathrm{~m}^{3}$ & $35 \mathrm{~m}^{2}$ \\
\hline Havešová & Slowakei & Korpel' 1995 & $3 \times 0,5$ ha & $655-773 \mathrm{~m}^{3}$ & $81-201 \mathrm{~m}^{3}$ & $32-36 m^{2}$ \\
\hline Kyjov & Slowakei & Text & $20 \times 0,39$ ha & $451 \mathrm{~m}^{3}$ & $110 \mathrm{~m}^{3}$ & $32 \mathrm{~m}^{2}$ \\
\hline Kyjov & Slowakei & Korpel' 1995 & $4 \times 0,5$ ha & $405-512 \mathrm{~m}^{3}$ & $63-66 \mathrm{~m}^{3}$ & $29-35 m^{2}$ \\
\hline Rožok & Slowakei & Korpel' 1995 & $3 \times 0,5$ ha* $^{*}$ & $577-794 \mathrm{~m}^{3}$ & $32-297 \mathrm{~m}^{3}$ & $32,6-44,3 \mathrm{~m}^{2}$ \\
\hline Stužica & Slowakei & Korpel' 1995 & $3 \times 0,5 \mathrm{ha}^{*}$ & $520-628 \mathrm{~m}^{3}$ & $71-149 \mathrm{~m}^{3}$ & - \\
\hline Puka & Albanien & Tabaku 1999 & 3,625 ha & $781 \mathrm{~m}^{3}$ & $32 \mathrm{~m}^{3}$ & $45,6 \mathrm{~m}^{2}$ \\
\hline Mirdita & Albanien & Tabaku 1999 & 5 ha & $559 \mathrm{~m}^{3}$ & $40 \mathrm{~m}^{3}$ & $37,2 \mathrm{~m}^{2}$ \\
\hline Rajca & Albanien & Tabaku 1999 & 6 ha & $807 \mathrm{~m}^{3}$ & $86 \mathrm{~m}^{3}$ & $43,4 \mathrm{~m}^{2}$ \\
\hline im Uholka-Massiv & Ukraine & Tsuryk 1980 & $2 \times 1$ ha & 588 u. $678 \mathrm{~m}^{3}$ & - & 40,2 u. $47,1 \mathrm{~m}^{2}$ \\
\hline im Uholka-Massiv & Ukraine & Commarmot 2005 & 10 ha & $770 \mathrm{~m}^{3}$ & $111 \mathrm{~m}^{3}$ & $38,5 \mathrm{~m}^{2}$ \\
\hline Uholka u. Berezniky & Ukraine & Chernavskyy 2005 & $21 \times 0,5-1$ ha & $463 \mathrm{~m}^{3}$ & $79 \mathrm{~m}^{3}$ & $35,9 \mathrm{~m}^{2}$ \\
\hline
\end{tabular}

Das Modell zur Simulation natürlicher Strukturen im Buchenwald von Neuert (1999) sollte deshalb an größere Holzvorräte angepasst werden. Seine Schätzung des Flächenanteils verschiedener Waldentwicklungsphasen und des mittleren Holzvorrats je Phase führt zu einem durchschnittlichen Bestandesvolumen von nur $360 \mathrm{~m}^{3} \mathrm{ha}^{-1}$.

Havešová erreicht einen Holzvorrat, wie er auch in den Tannen-Buchen-Urwaldreservaten Čorkova Uvala in Kroatien (Neumann 1979) oder Peručica in Bosnien (Leibundgut 1993) stockt. Im Tannen-Buchen-Urwald Dobroč in der Slowakei schwankt der Vorrat auf 0,5 ha großen Flächen zwischen 550 und $1120 \mathrm{~m}^{3} \mathrm{ha}^{-1}$ (Saniga 1999). Der durchschnittliche Holzvorrat im Tannen-Buchen-Urwaldreservat Rothwald in Österreich (Neumann 1979) entsprach dagegen dem Vorrat in Kyjov. Holzvorräte über $1000 \mathrm{~m}^{3} \mathrm{ha}^{-1}$ werden in den 
untersuchten Buchen-Urwaldreservaten nur auf kleiner Teilfläche und auch nicht in jedem Reservat erreicht.

Der durchschnittliche Totholzvorrat in Havešová und Kyjov war größer als in albanischen Buchen-Urwaldreservaten, deren Vorräte im Mittel zwischen 31 und $86 \mathrm{~m}^{3} \mathrm{ha}^{-1}$ lagen (Tabaku 1999). Nach Saniga und Schütz (2002) schwankten in den slowakischen BuchenUrwaldreservaten Havešová, Rožok und Stužica die Vorräte auf 0,5 ha großen Flächen zwischen 25 und $300 \mathrm{~m}^{3} \mathrm{ha}^{-1}$. Der Durchschnitt beträgt ungefähr $130 \mathrm{~m}^{3} \mathrm{ha}^{-1}$. Diese Werte stimmen mit den Ergebnissen unserer Untersuchung gut überein. Lediglich die Maximalwerte wurden nur auf kleinerer Fläche erreicht. Korpel' (1995) ermittelte in Kyjov im Jahr 1983 einen Totholzvorrat von $65 \mathrm{~m}^{3} \mathrm{ha}^{-1}$. Neben methodischen Unterschieden bei der Flächenauswahl gibt die Differenz zu unseren Ergebnissen auch Hinweise auf verstärkte Störungen in den letzten 20 Jahren. Chernyavskyy (2005) gibt für einen Urwald in der Ukraine einen über mehrere Phasen gemittelten Vorrat von $75 \mathrm{~m}^{3} \mathrm{ha}^{-1}$ an. Als Fazit lässt sich für das mittlere Totholzvolumen eine große Streuung zwischen den Beständen festhalten: je nach Wüchsigkeit und natürlichen Störungseinflüssen können die Durchschnittswerte 30 bis $130 \mathrm{~m}^{3} \mathrm{ha}^{-1}$ betragen. Die große Streuung wird in erster Linie durch unterschiedliche Standorte verursacht, auf denen unterschiedlich hohe Bäume mit unterschiedlichem Holzvolumen wachsen. Im Durchschnitt lag der Anteil des Totholzes am Gesamtholzvorrat etwas über $10 \%$, ein Anteil über $20 \%$ wurde in keinem der untersuchten Urwaldreservate erreicht. Müller (2005) schlägt $40 \mathrm{~m}^{3}$ starkes Totholz pro Hektar als Richtwert im Wirtschaftswald vor, Ammer (1991) 15-30 $\mathrm{m}^{3} \mathrm{ha}^{-1}$. Das würde ungefähr 20-50\% des Totholzvorrats in Buchen-Urwäldern entsprechen. Zahlreiche Diskussionspunkte zu diesem Thema spricht Richter (2000) an und verweist auf 9 bis $21 \mathrm{~m}^{3} \mathrm{ha}^{-1}$ als Empfehlung von Korpel' (1995) für Wirtschaftswälder. Ein von der Bedeutung für xylobionte Käfer abgeleitetes Verhältnis von 1:1 an liegendem und stehendem Totholz von Ammer (1991) stimmt nicht mit dem vorgefundenen Verhältnis in den slowakischen Buchenurwäldern überein. Der Anteil an liegendem Totholz war drei- bis viermal höher. Auf Grund der Abhängigkeit des Totholzvolumens von der Bestandesoberhöhe sollten Richtwerte für den Totholzanteil in bewirtschafteten Wäldern die Höhenwuchsleistung mitberücksichtigen.

Die Unterschiede im Derbholzvorrat zwischen den beiden untersuchten Reservaten sind in erster Linie auf unterschiedliche Baumhöhen bzw. den Standort zurückzuführen. Ein Vergleich der Grundfläche führte zu keinen deutlichen Unterschieden zwischen den Reservaten. Korpel' (1995) ermittelte auf 0,5 ha großen Flächen in beiden Reservaten 
Grundflächen, die zwischen 29 und $36 \mathrm{~m}^{2} \mathrm{ha}^{-1}$ schwankten. Tabaku (1999) ermittelte in den drei albanischen Buchen-Urwaldreservaten Grundflächen von 37 bis $46 \mathrm{~m}^{2} \mathrm{ha}^{-1}$. Die zwei wüchsigeren Reservate in Albanien besitzen Grundflächen, die auch in Tannen-BuchenUrwäldern (Neumann, 1979) erreicht werden. In den untersuchten Reservaten in der Slowakei werden diese Werte nur in Ausnahmefällen auf den 0,4 ha großen Probeflächen erreicht. Commarmot et al. (2005) ermittelten auf 0,25 ha großen Unterflächen Grundflächen von 25 bis $50 \mathrm{~m}^{2} \mathrm{ha}^{-1}$, die im Durchschnitt $38,5 \mathrm{~m}^{2}$ ha $^{-1}$ betrugen. Je nach Standort kann man dementsprechend in einem Buchenurwald mittlere Grundflächen von 30 bis rund $45 \mathrm{~m}^{2} \mathrm{ha}^{-1}$ annehmen.

Die slowakischen und zwei der albanischen Reservate ähneln sich in der Anzahl der Bäume pro Hektar (durchschnittlich 289 bis 331 Bäume, siehe Tabaku 1999). Nur das albanische Reservat Rajca besitzt mit 391 Bäumen ha ${ }^{-1}$ eine höhere Dichte, die in dem häufigeren Auftreten schwacher Bäume begründet ist. Korpel' (1995) gibt für Havešová etwa 225 und für Kyjov ungefähr 350 Bäume ha ${ }^{-1}$ an.

Die Ansprache der räumlichen Struktur auf den einzelnen Probeflächen durch den strukturellen Komplexitätsindex nach Zenner und Hibbs (2000) zeigte nur geringen Einfluss auf Holzvorrat, Bestandesdichte oder Stammzahl. Während in Kyjov der Vorrat und die Grundfläche mit zunehmend heterogener Struktur etwas anstieg, war in Havešová keine Tendenz zu erkennen.

Die Durchmesserverteilungen in Havešová und im albanischen Reservat Rajca wiesen einen ausgeprägten exponentiellen Verlauf auf. Westphal et al. (2006) stellten unterschiedliche Durchmesserverteilungen in 9 Buchenurwäldern (einschließlich Havešová und Kyjov mit Daten aus dieser Untersuchung) fest. In Kyjov tritt eine vergleichsweise geringe Anzahl Bäume mit 7-11 cm BHD und eine große Anzahl mit 12-20 cm BHD auf. Eine mögliche Ursache für dieses Ungleichgewicht können Kahlschläge darstellen, die vor 2-3 Jahrzehnten in unmittelbarer Umgebung des 53 ha großen Reservats durchgeführt wurden und verstärkt zu flächigen Windwürfen geführt haben. Die Verjüngung dieser Windwurfflächen stellt heute wahrscheinlich einen großen Teil der Bäume in der übermäßig stark besetzten 12-20 cm BHD Klasse dar. Ein anderer Grund kann verstärkter Eisbruch im Gebiet von Kyjov nach dem Bau eines Stausees vor 30 Jahren sein, vom dem ortsansässige Förster berichten. Diese Überlegungen machen deutlich, dass bereits ein Reservat der Größe wie Kyjov durch anthropogene Tätigkeiten außerhalb des Reservats gestört werden kann. 
Der maximale Durchmesser in Kyjov wurde dagegen nicht beeinflusst und liegt, wie in den anderen Reservaten auch, über $110 \mathrm{~cm}$ (nur für Mirdita in Albanien werden $99 \mathrm{~cm}$ berichtet, siehe Tabaku 1999).

Die Baumhöhen für $80 \mathrm{~cm}$ starke Buchen schwankten in den betrachteten fünf slowakischen und albanischen Urwaldreservaten zwischen 33 und 47 m (Tabaku 1999). Danach besitzt Havešová die beste Höhenbonität, während Kyjov und Mirdita die schlechteste aufweisen. Der Kronenansatz der Bäume in der Oberschicht lag in Kyjov und den albanischen Reservaten in ungefähr 10-13 m Höhe. In Havešová liegt der Kronenansatz in 15-20 m Höhe. Der durchschnittliche Anteil der Kronenlänge an der Baumhöhe liegt in der Oberschicht in den fünf betrachteten Reservaten bei 56 bis 61\%. Der Schlankheitsgrad der Bäume betrug 60 bis 67. Die Kronenschirmfläche eines $60 \mathrm{~cm}$ starken Baumes nahm in allen Reservaten durchschnittlich $125 \mathrm{~m}^{2}$ ein. Extrem ausladende Kronen überschirmten bis zu $300 \mathrm{~m}^{3}$ (Tabaku 1999).

\subsection{ZUSAMMENFASSUNG}

In zwei Buchen-Urwaldreservaten der montanen Stufe in der Ostslowakei (Havešová und Kyjov) wurden durch eine Stichprobeninventur insgesamt 16,4 ha Waldfläche voll aufgenommen. Das Reservat Havešová weist sehr gute und das Reservat Kyjov durchschnittliche Höhenwuchsleistungen auf. Die Buche bildete auf beiden Standorten Reinbestände. Das Wasser- und Nährstoffangebot, die Zusammensetzung der Bodenvegetation und die im Reservat Havešová geprüfte genetische Ausstattung ließen keine großen Unterschiede im Vergleich mit deutschen Buchenwirtschaftswäldern erkennen.

Die Verjüngung setzte sich in beiden Reservaten trotz unterschiedlichen Lichtangebots von im Mittel 2,5 \% in Havešová und 9,4 \% in Kyjov (Diffuse Site Factor am Waldboden) aus rund 70.000 Pfl. ha ${ }^{-1}$ zusammen. Buchenpflanzen dominierten die Verjüngung,

Nebenbaumarten besaßen aber sehr große Anteile an Pflanzen < 0,5 m Höhe. Die Verjüngung war ohne erkennbaren Einfluss der unterschiedlichen Lichtverhältnisse in beiden Reservaten auf $90 \%$ der Fläche vorhanden.

In Havešová stockte ein durchschnittlicher Holzvorrat von $720 \mathrm{~m}^{3} \mathrm{ha}^{-1}$, in Kyjov von $450 \mathrm{~m}^{3}$ ha $^{-1}$. Kleinflächig schwankten die Werte zwischen 100 und $1000 \mathrm{~m}^{3} \mathrm{ha}^{-1}$. Das Totholzvolumen betrug $130 \mathrm{~m}^{3} \mathrm{ha}^{-1}$ bzw. $110 \mathrm{~m}^{3} \mathrm{ha}^{-1}$, was einem Fünftel des lebenden Vorrats entspricht. In beiden Reservaten standen ungefähr 300 Bäume mit mindestens $7 \mathrm{~cm}$ BHD auf einem Hektar. Sie bildeten eine Grundfläche von $35 \mathrm{~m}^{2} \mathrm{ha}^{-1}$ in Havešová und $32 \mathrm{~m}^{2}$ $\mathrm{ha}^{-1}$ in Kyjov. 
Die Bäume der Oberschicht besaßen einen Schlankheitsgrad von 60 bzw. 67. Die Kronenschirmfläche von Bäumen mit $60 \mathrm{~cm}$ BHD betrug rd. $125 \mathrm{~m}^{2}$.

Zusammenhänge zwischen Bestandesdichte und Bestandesstruktur (ausgedrückt durch den Komplexitätsindex nach Zenner und Hibbs, 2000) und zwischen Bestandesdichte und Stammqualität ließen sich nicht erkennen.

\subsection{Summary}

Title: Stand structure, regeneration and site conditions in two virgin beech forest reserves in Slovakia.

The study was conducted in the virgin beech forest reserves Havešová and Kyjov in eastern Slovakia. Both reserves are located in the montane region and represent pure beech stands with less than $1 \%$ other tree species. The Havešová reserve showed a very good height growth (max. tree height $>50 \mathrm{~m}$ ) and the Kyjov reserve an intermediate height growth (max. tree height $34 \mathrm{~m}$ ). The base rock in Havešová is flysch and in Kyjov andesit. Both reserves comprise cambisol soils.

An important aim of the study was to determine the amount of standing volume and deadwood volume in virgin beech forests. Therefor a systematic sample inventory was carried out on an area of together 16.4 ha. In Havešová 22 sample plots and in Kyjov 20 sample plots á $62.5 \mathrm{~m} \mathrm{x} 62.5 \mathrm{~m}$ were established and trees with $\mathrm{dbh} \geq 7 \mathrm{~cm}$ and deadwood with diameter $\geq$ $20 \mathrm{~cm}$ and lenght $\geq 2 \mathrm{~m}$ were measured.

In addition the water and nutrient supply and the composition of herb vegetation as well as genetic features were analysed to assess the comparability to other beech stands. In general these features did not indicate large differences to managed beech stands in Germany. The genetic distance of seed from Havešová and seed from northwestern Germany amounted to $10 \%$ approximately. Seedlings from Slovakia started to unfold their leaves 3-4 days earlier than German seedlings. An important difference to western Europe is the more continental climate, which is characterized by $20^{\circ} \mathrm{C}$ mean temperature in july and $-3{ }^{\circ} \mathrm{C}$ in january. However, this amplitude of temperature is no limitation for the growth of beech. The annual precipitation (700-800 $\mathrm{mm}$ ) and soil conditions (depth at least $1 \mathrm{~m}$, with a high proportion of clay and silt) ensure a good water supply. A normal storage of nutrients was found. Only the carbon storage in Havešová and the magnesium storage in Kyjov was low compared to criteria of the forest soil inventory in Germany. However, no lack of elements was found. The ratio of carbon and nitrogen amounted to 10 and indicated a high decomposition rate. 
Ecological indicators derived from the vegetation describe the temperature as cool to moderately warm and the humidity as fresh. The ecotype of both beech forests is the Dentario glandulosae-Fagetum.

In spite of different light conditions (2.5\% respectively $9.4 \%$ diffuse site factor) in both reserves the density of forest regeneration amounted to 70.000 trees $^{-1} \mathrm{a}^{-1}$. It was dominated by beech, only the seedling height class $<0.5 \mathrm{~m}$ containing a significant proportion of mixed tree species. In both reserves the regeneration occured on $90 \%$ of the area independently from light conditions.

The first reserve had a mean standing volume of $720 \mathrm{~m}^{3} \mathrm{ha}^{-1}$. On the single 22 plots the volume amounted from 450 to $960 \mathrm{~m}^{3} \mathrm{ha}^{-1}$. The second reserve had a mean standing volume of $450 \mathrm{~m}^{3} \mathrm{ha}^{-1}$. It amounted from 350 to $550 \mathrm{~m}^{3} \mathrm{ha}^{-1}$ on the plots. On small patches of 2.500 $\mathrm{m}^{2}$ the volume ranged from 100 to $1000 \mathrm{~m}^{3} \mathrm{ha}^{-1}$.

Deadwood was determined with $130 \mathrm{~m}^{3} \mathrm{ha}^{-1}$ respectively $110 \mathrm{~m}^{3} \mathrm{ha}^{-1}$ (approximately one fifth of live timber volume). In Havešová it amounted from 33 to $286 \mathrm{~m}^{3} \mathrm{ha}^{-1}$ and in Kyjov from 46 to $176 \mathrm{~m}^{3} \mathrm{ha}^{-1}$ on the sample plots.

300 trees ha ${ }^{-1}$ were counted and comprised a basal area of $35 \mathrm{~m}^{2} \mathrm{ha}^{-1}$ in Havešová and $32 \mathrm{~m}^{2}$ $\mathrm{ha}^{-1}$ in Kyjov. On the plots the tree number amounted from 200 to 400 trees $^{-1}$ and the basal area from 25 to $45 \mathrm{~m}^{2} \mathrm{ha}^{-1}$.

The ratio of height to dbh amounted to 60 and 67 for canopy trees. The crown cover of a tree with $60 \mathrm{~cm}$ dbh reached as an average $125 \mathrm{~m}^{2}$. A relation between stand density and stand structure (described by the complexity index of Zenner and Hibbs, 2000) or between stand density and stem quality could not be observed.

\subsection{Literatur}

Ammer, U.: Konsequenzen aus den Ergebnissen der Totholzforschung für die forstliche Praxis. Forstw. Cbl. 110: 149-157.

Bohn, U., Neuhäusl, R., Gollub, G., Hettwer, C., Neuhäuslova, Z., Schlüter, H., Weber, H.: Karte der natürlichen Vegetation Europas. Teil 1: Erläuterungstext. S.321. Landwirtschaftsverlag. Münster, 2003

Braun-Blanquet, J.: Pflanzensoziologie. Springer Verlag. Wien, New York. 3. Auflage. 1964

Brumme, R., Egenolf, M., Riek, W., Aydin, C., Block, J., Lohmann, H., Meiwes, K. J., Wilpert, K. v., Wolff, B.: Soil organic carbon and nitrogen stocks and control in forest soils of Germany. In: Brumme, R. Khanna, P. K. (eds): Functioning and management of European beech ecosystems. Ecological Studies. Springer Verlag Berlin, Heidelberg, New York, 2006 (in press)

Wolff, B. u. Riek, W.: Deutscher Waldbodenbericht 1996. Bd. 2. BMELF Bonn, 1997

Chernyavskyy, M.: The dynamics of virgin beech forests in the Ukrainian Carpathians. In: Hamor, F.D. and Commarmot, B. (eds): Natural forests in the temperate zone of Europe - Values and utilisation. International conference in Mukachevo, Ukraine. October 13-17, 2003. Rakhiv, Carpathian biosphere Reserve; Birmensdorf, Swiss Federal Research Institute WSL.

Commarmot, B., Bachofen, H., Bundziak, Y., Bürgi, A., Ramp, B., Shparyk, Y., Sukhariuk, D., Viter, R. und Zingg, A. 2005. Structures of virgin and managed beech forests in Uholka (Ukraine) and Sihlwald (Switzerland): A comparative study. For. Snow Landsc. Res. 79: 45-56, 2005

Czajkowski, T., Bolte, A.: Unterschiedliche Reaktion deutscher und polnischer Herkünfte der Buche (Fagus sylvatica L.) auf Trockenheit. Allg. Forst- u. J.-Ztg. 177: 30-40, 2006 
Czajkowski, T., Köhling, M., Bolte, A.: Zur Verbreitungsgrenze der Buche (Fagus sylvatica L.) im nordöstlichen Mitteleuropa. Forstarchiv, 2006 (in press)

De Candolle, A.: Geographie Botanique raisonée. Paris et Genévé, 1855

Diaci, J., Thormann, J.-J.: Ein Vergleich verschiedener Lichtmessmethoden in Buchennaturwäldern Sloweniens aus verjüngungsökologischer Sicht. Schweiz. Z. Forstwes. 153: 39-50, 2002

Dierschke, H.: Zur Aufnahme und Darstellung phänologischer Erscheinungen in Pflanzengesellschaften. In: Tüxen, R. (Ed.): Grundlagen und Methoden in der Pflanzensoziologie. Berichte vom Int. Symp. IXV in Rinteln 1970: 291-311, 1972

Dierschke, H.: Artenreiche Buchenwald-Gesellschaften Nordwest-Deutschlands. Ber. D. Reinh. Tüxen-Ges. 1, 107-148. Göttingen, 1989

Drößler, L., Lüpke, B. v.: Canopy gaps in two virgin beech forest reserves in Slovakia. Journal of Forest Science 51: 446-457, 2005

Drößler, L., Meyer, P.: Waldentwicklungsphasen in zwei Buchen-Urwaldreservaten in der Slowakei. Forstarchiv 77: 155-161, 2006

Ellenberg, H.: Vegetation Mitteleuropas mit den Alpen. Ulmer Verlag. Stuttgart, 1996

Ellenberg, H.: Zeigerwerte von Pflanzen in Mitteleuropa. Goltze Verlag. Göttingen, 2001

Hattemer, H.H., Bergmann, F., Ziehe, M.: Einführung in die Genetik für Studierende der Forstwissenschaft. Sauerländer's Verlag. Frankfurt, 1993

Hertel, D.: Das Feinwurzelsystem von Rein- und Mischbeständen der Rotbuche: Struktur, Dynamik und interspezifische Konkurrenz. Dissertationes Botanicae, Band 317, 1999

Hoffmann, D.: Wasser- und Lufthaushalt des Bodens. In: Forstliche Standortsaufnahme. IHW-Verlag. Eching, 1996

Jaloviar, P.: Biomasa a nekromasa jemných koreňov v bukovom prírodnom lese Havešová. Vedecké štúdie, TU Zvolen, Slowakisch (in press), 2005

Konnert, M., Ziehe, M., Tröber, U., Maurer, W., Janßen, A., Sander, T., Hussendörfer, E., Hertel, H.: Genetische Variation der Buche (Fagus sylvatica L.) in Deutschland: Gemeinsame Auswertung genetischer Inventuren über verschiedene Bundesländer. Forst und Holz 55, 403-408, 2000

Korpel', Š.: Die Urwälder der Westkarpaten. G. Fischer Verlag. Stuttgart, Jena, New York, 1995

Leibundgut, H.: Europäische Urwälder. Verlag Paul Haupt. Bern, Stuttgart, 1993

Le Tacon, F.: Caracterisation edaphique. In : Teissier du Cros, E. La Hêtre. 77-84, INRA, Paris, 1981

Leuschner, C., Rode, M., Heinken, T.: Gibt es eine Nährstoffmangel-Grenze der Buche im nordwestdeutschen Flachland? Flora 188: 239-249, 1993

Leuschner, C.: Das Konzept der potentiellen natürlichen Vegetation (PNV): Schwachstellen und Entwicklungsperspektiven. Flora 192: 379-391, 1997

Leuschner, C.: Mechanismen der Konkurrenzüberlegenheit der Rotbuche. Ber. d. Reinh.-Tüxen-Ges. 10: 5-18, 1998

Mayer, H.: Waldbau auf soziologisch-ökologischer Grundlage. Fischer Verlag, Stuttgart, New York, 1984

Mühr, B.: URL http://www.klimadiagramme.de/Europa/uzgorod.html (abgerufen am 29.5.06), 2002

Müller, J.: Waldstrukturen als Steuergröße für Artengemeinschaften in kollinen bis submontanen

Buchenwäldern. Diss. TU München, 2005

Müller, T.: Die artenreichen Rotbuchenwälder Süddeutschlands. Ber. D. Reinh. Tüxen-Ges. 1, 149-163.

Göttingen, 1989

Mund, M.: Carbon pools of European beech forests (Fagus sylvatica) under different silvicultural management. Ber. d. Forschungszentrums Waldökosysteme, Reihe A, Bd. 189, 2004

Neuert, C.: Die Dynamik räumlicher Strukturen in naturnahen Buchenwäldern Mitteleuropas. UFZ-Bericht Nr. 20/1999

Neumann, M.: Bestandesstruktur und Entwicklungsdynamik im Urwald Rothwald/NÖ und im Urwald Čorkova Uvala/Kroatien. Diss. Univ. f. Bodenkultur, Wien, 1979

Petráš, R. und Pajtík, J., Sústava Česko-slovenských objemových tabuliek drevín. Lesnícky Časopis 37: 49-56. Slowakisch, 1991

Pichler, V., Gregor, J., Bebej, J.: Retenèné krivky vybraných lesných pôd Slovenska. LF TU Zvolen.

Slowakisch, 2005

Richter, J.: Wie naturnah kann mitteleuropäische Forstwirtschaft sein? Schriftenr. der Landesforstverw. NRW, Heft 12, 2000

Röhrig, E., Bartsch, N, Lüpke, B. v.: Waldbau auf ökologischer Grundlage. Ulmer Verlag, Stuttgart, 2006 Saniga, M.: Štruktúra, produkčné a regeneračné procesy Dobročského pralesa. Vedecké štúdie 2/1999/A, TU Zvolen, Slowakisch, 1999

Saniga, M., Schütz, J.P.: Relation of dead wood course within the development cycle of selected virgin forests in Slovakia. Journal of Forest Science 48: 513-528, 2002

Schmidt, W.: Untersuchungen über die Phosphorversorgung niedersächsischer Buchenwaldgesellschaften.

Scripta Geobotanica Band 1, 1970 
Schöning, I.: FORCAST (Forest Carbon - Nitrogen Trajectories) data base.

URL http://www.dow.wau.nl/natcons/NP/FORCAST/files_database_forecast2.htm (abgerufen am 6.7.06), 2003

Tabaku, V.: Struktur von Buchen-Urwäldern in Albanien im Vergleich mit deutschen Buchen-

Naturwaldreservaten und -Wirtschaftwäldern. Diss. Univ. Göttingen, 1999

Tabaku, V., Meyer, P.: Lückenmuster albanischer und mitteleuropäischer Buchenwälder unterschiedlicher Nutzungsintensität. Forstarchiv 70: 87-97, 1999

Tarasiuk, S.: Buk zwyczajny (Fagus sylvatica L.) na obrzeżach zasięgu w Polsce. Diss. Univ. Warzsawa, 1999

Tsuryk, J.E.: Struktura i vosobnowlenie devstvennyh bukovyh drevostojev Karpat. Lesovedenie 5: 75-84.

Russisch, 1980

Višnjic, C., Dohrenbusch, A.: Frostresistenz und Phänologie europäischer Buchenprovenienzen (Fagus sylvatica L.). Allg. Forst- u. J.-Ztg., 175: 101-108.

Wagner, S.: Strahlungsschätzung in Wäldern durch hemisphärische Fotos - Methode und Anwendung. Berichte des Forschungszentrums Waldökosysteme, Göttingen. Reihe A 123: 1-166, 1994

Westphal, C., Tremer, N., Oheimb, G. v., Hansen, J., Gadow, K. v., Härdtle, W.: Ist the reverse J-shaped diameter distribution universally applicable in Eurpean virgin beech forests? Forest Ecology and Management 223: 75-83, 2006

Willkomm, M.: Forstliche Flora von Deutschland und Österreich. Winter Verlag, Leipzig, 1887

Zeibig, A., Diaci, J., Wagner, S.: Gap disturbance patterns of a beech virgin forest remnant in the mountain vegetation belt of Slovenia. In: Hamor, F.D., Commarmot, B. (eds.), Natural forests in the temperate zone of Europe - Values and utilisation. International Conference in Mukachevo, Ukraine. October 13-17, 2003. Rakhiv, Carpathian Biosphere Reserve; Birmensdorf, WSL, 2005

Zenner, E.K., Hibbs D.E.: A new method for modeling the heterogeneity of forest structure. Forest Ecology and Management 129: 75-87, 2000

Zolyomi, B., Barath, Z., Fekete, G., Jakucs, P., Karpati, I., Karpati, V., Kovacs, M., Mate, I.: Einreihung von 1400 Arten der ungarischen Flora in ökologische Gruppen nach TWR-Zahlen. Fragmenta Bot. Mus. Hist. Nat. Hung. 4: 101-142, 1966 


\section{Stand structure in virgin and managed beech forests}

Dieser Teil entspricht einem eingereichten Manuskript für das European Journal of Forest Research mit dem Titel „Stand structure in virgin and managed beech forests“ von L. Drößler und B. v. Lüpke

\subsection{Abstract}

The study focuses on stand structure in two virgin beech forests in Slovakia and six relatively intensively managed forests in Germany. The objective is to test several structural parameters in order to find out the most suitable one for describing differences between natural and managed beech forests and to aid management decisions aiming at close-to-nature forestry. Different structural indicators are calculated and their meaningfulness is assessed.

Firstly, a very detailed structural analysis was undertaken using tree coordinates, stem diameter and tree crown radius data. Neighbouring trees of similar sizes were regarded as a group and the number and size of groups were determined. As a result in each stand the majority of such groups consisted of single trees. The frequency of larger tree groups decreased exponentially. This grouping summarized data about complex forest structure in a comprehensive way for silvicultural applications. It demonstrated for virgin forests smaller patches in the average than in even aged forests. However, the smallest patches were found in selection forests (Plenterwald). Comparisons of forest types could be drawn by absolute tree numbers of groups.

Secondly, other indicators like diameter distribution, diameter differentiation and a structural complexity index were calculated. All indicators allowed differentiation between the stands. Diameter distributions were helpful for management decisions, because they could suggest removals of trees by diameter. The diameter differentiation and the structural complexity index both resulted in one relative value per stand. Thus the most heterogenously structured stands could be determined however, no management conclusions could be drawn.

Concerning silvicultural decisions in forest practice, we recommend diameter distributions due to the low input in data survey. For more detailed investigations concerning stand structure we recommend the spatial grouping of trees of similar size.

Keywords: Forest structure, heterogeneity, spatial pattern, close-to-nature forestry, Fagus sylvatica 


\subsection{Introduction}

Forest structure has always played a central role in forest descriptions. More recently this broadly used term has gained importance in discussions about close-to-nature forestry. In Germany, this is a widely accepted management goal, at least in forests owned by the public. To achieve this goal in an optimal way two points are of basic importance: knowledge about the structure of natural forests, and adequate methods to describe this structure. The last point should enable forest management to control silvicultural decisions, and to assess how much the actual silvicultural situation differs from natural conditions. In both respects aspects information is scarce. Through the following investigation we want to narrow this knowledge gap for beech forests as the most important objects of close-to-nature forestry in Germany.

There are some intensive investigations comparing forests managed under different close-tonature regimes (Kern, 1966; Schrempf, 1986; Tabaku, 1999). They focus on stand data such as density, crown parameters and diameter distribution. Besides other output they produced crown cover maps providing valuable information about the arrangement of trees. Yet it is difficult to analyse complex forest structures on large representative areas. Schrempf (1986) and Tabaku (1999) additionally identified forest development stages to describe the horizontal forest structure. However, the boundaries of these stages are not always clearly detectable in the field, and often arbitrary decisions have to be taken. Tabaku's (1999) classification of forest development stages used an objective computer based method developed by Meyer (1999). The whole stand was divided in $12.5 \mathrm{~m} \mathrm{x} 12.5 \mathrm{~m}$ plots, and each plot was assigned one development stage. Plot size approximates the area an old dominant beech tree crown can cover. Yet the arrangement of trees does not always follow this pattern. Stages can overlap as shown by the differentiation of development stages in a selection forest by Grassi et al. (2003).

Our observations in virgin forests indicated that a development stage can comprise only of one tree. This led us to the idea of defining development stages by groups of neighbouring trees of approximately the same size. By this, we regarded forests as a mixture of such groups. We hypothesized that the frequency of groups of every particular tree size will decrease exponentially as group size (in terms of tree numbers per group) increases. Canopy gaps in these forests, e.g. show exponentially decreasing size distributions (Drößler and Lüpke, 2005). Therefore, later stages might also occupy many small areas and few large areas.

In a second step we compared this stand structure indicator with other indicators and tested whether they were suitable to distinguish between forests of different degrees of naturalness, ranging from a classical uniform shelterwood to virgin forests. Information about structural 
indicators and their derivations is available in Pommerening (2002) with a particular focus on diameter differentiation, and Zenner and Hibbs (2000) on the structural complexity index.

Our study considers mainly horizontal tree distribution, tree diameter, tree height, and the mean crown radius of live trees. Thus it excludes many other important elements of forest structure such as dead wood (see e.g. Winter et al., 2003). However, setting this limit simplified the task, which may be justified as a step towards the development of a silviculturally useful indicator capable of describing the degree of naturalness of forest structures.

\subsection{Field Sites}

The main investigation was carried out in two virgin forest reserves in Eastern Slovakia. In the Havešová Reserve (170 ha) 22 systematically distributed sample plots of 0.4 ha were established (Table 14). In the second reserve Kyjov (50 ha) 20 sample plots of the same size were recorded. After the sampling in Kyjov one large plot (13 ha with 4.112 trees) was established to determine tree groups by additional measurement of trees between the original sample plots.

Both reserves comprise of pure beech forests with almost no admixed tree species (less than one percent). Stand top height is approximately $45 \mathrm{~m}$ in Havešová and $30 \mathrm{~m}$ in Kyjov. Additional stand information is available in Korpel' (1995) and Drößler and Lüpke (2006).

Table 14. Description of the stands investigated

\begin{tabular}{|c|c|c|c|c|c|c|c|c|}
\hline Stand & $\begin{array}{c}\text { Havešová } \\
\text { (HAV) }\end{array}$ & $\begin{array}{l}\text { Kyjov } \\
\text { (KYJ) }\end{array}$ & $\begin{array}{c}\text { Bleicherode } \\
\text { (UA1) }\end{array}$ & $\begin{array}{c}\text { Dörna } 2 \\
\text { (UA2) }\end{array}$ & $\begin{array}{c}\text { unit 148a } \\
(Y 86)\end{array}$ & Y120 & $\begin{array}{c}\text { Stauffenburg } \\
(Y 166)\end{array}$ & $\begin{array}{c}\text { Limker Strang } \\
(\mathrm{Y} 148)\end{array}$ \\
\hline Age & uneven & uneven & uneven & uneven & 86 years & 120 years & 166 years & 148 years \\
\hline $\begin{array}{l}\text { Type of } \\
\text { Management }\end{array}$ & $\begin{array}{l}\text { virgin forest } \\
\text { reserve }\end{array}$ & $\begin{array}{l}\text { virgin forest } \\
\text { reserve }\end{array}$ & $\begin{array}{l}\text { single tree } \\
\text { selection }\end{array}$ & $\begin{array}{l}\text { single tree } \\
\text { selection }\end{array}$ & $\begin{array}{l}\text { heavy } \\
\text { thinning }\end{array}$ & $\begin{array}{l}\text { heavy } \\
\text { thinning }\end{array}$ & $\begin{array}{l}\text { target } \\
\text { diameter } \\
\text { cutting }\end{array}$ & $\begin{array}{l}\text { reserve for } \\
25 \text { years }\end{array}$ \\
\hline $\begin{array}{l}\text { base rock } \\
\text { soil type }\end{array}$ & $\begin{array}{l}\text { sandstone } \\
\text { cambisol }\end{array}$ & $\begin{array}{l}\text { andesite } \\
\text { cambisol }\end{array}$ & $\begin{array}{l}\text { limestone } \\
\text { rendzina }\end{array}$ & $\begin{array}{l}\text { limestone } \\
\text { rendzina }\end{array}$ & $\begin{array}{l}\text { limestone } \\
\text { rendzina }\end{array}$ & $\begin{array}{l}\text { limestone } \\
\text { rendzina }\end{array}$ & $\begin{array}{l}\text { sandstone } \\
\text { cambisol }\end{array}$ & $\begin{array}{l}\text { sandstone } \\
\text { cambisol }\end{array}$ \\
\hline Tree species & F. sylvatica & F. sylvatica & F. sylvatica & $\begin{array}{l}\text { F. sylvatica } \\
\text { A. pseudoplat. } \\
\text { A. platanoides } \\
\text { Tilia cordata }\end{array}$ & $\begin{array}{l}\text { F. sylvatica } \\
\text { A. pseudoplat. } \\
\text { F. excelsior }\end{array}$ & F. sylvatica & F. sylvatica & F. sylvatica \\
\hline $\begin{array}{l}\text { top stand } \\
\text { height }\end{array}$ & $45 \mathrm{~m}$ & $30 \mathrm{~m}$ & $33.5 \mathrm{~m}$ & $31.0 \mathrm{~m}$ & $31.2 \mathrm{~m}$ & $35.9 \mathrm{~m}$ & $38.4 \mathrm{~m}$ & $35.6 \mathrm{~m}$ \\
\hline $\begin{array}{l}\text { number of } \\
\text { plots and size }\end{array}$ & $22 \times 0.4$ ha & $20 \times 0.4$ ha & $1 \times 7.5$ ha & $1 \times 1.5$ ha & $1 \times 0.4$ ha & $1 \times 0.4$ ha & $1 \times 7.25$ ha & $1 \times 8.75$ ha \\
\hline Tree numbers & 2496 & 2503 & 1887 & 650 & 215 & 116 & 663 & 1442 \\
\hline described in & Korpel' (1995) & Korpel' (1995) & Tabaku (1999) & Drößler (2001) & $\begin{array}{c}\text { Kahle et al. } \\
\text { (1996) }\end{array}$ & unpubl. data & Tabaku (1999) & Tabaku (1999) \\
\hline
\end{tabular}

Additionally, six managed forests in Central Germany were inventoried for comparison (Table 14): two uneven aged forests (UA1 and UA2), and four even aged forests between 86 
and 166 yrs. old (Y86, Y120, Y148, Y166). Y86 and Y120 have been managed during the last 30-40 years in the conventional way, i.e. heavy thinning until ca. 110 years, followed by the use of the uniform shelterwood system. Y148, initially also a conventionally managed stand, was classified as a forest reserve 25 years ago. Y166 has been harvested under a target diameter cutting regime for several decades. The data of these stands (mostly provided by other authors) were obtained from one representative part of each stand, with areas between 0.4 and 8.75 ha. Although a measured area of 0.4 ha appears to be rather small, the stands Y86 and Y120 were included in the study as their structure was assessed visually as sufficiently homogeneous.

\subsection{Methods}

\subsubsection{Tree groups}

In the study plots the Cartesian coordinates of the tree locations and diameters at breast height $(\mathrm{DBH})$ of the trees were measured. All living trees with $\mathrm{DBH} \geq 7 \mathrm{~cm}$ were recorded. In each stand, tree height and eight crown radii were measured for at least 30 trees that were evenly distributed across the diameter spectrum. The mean crown radius of a tree was calculated from the eight radii measured. A good linear correlation between DBH and mean crown radius were obtained, with stand specific regression lines and $\mathrm{R}^{2}$ values between 0.7 and 0.9 (see Tabaku 1999, Drößler and Lüpke 2006). Thus, for every tree with known DBH the mean crown radius could be estimated and a circular crown be allocated. The relationship between DBH and tree height could also be described by functions (see Tabaku, 1999, p. 61 and Drößler and Lüpke, 2005), and with known DBH, the height of a tree could be estimated.

Table 15. Developmental phases as defined for managed forests in Germany

\begin{tabular}{ll} 
DBH class & Description \\
\hline $4-6 \mathrm{~cm}$ & Thicket \\
$7-15 \mathrm{~cm}$ & Sapling stand \\
$16-20 \mathrm{~cm}$ & Pole stand \\
$21-34 \mathrm{~cm}$ & Small timber stand \\
$35-49 \mathrm{~cm}$ & Middle timber stand \\
$50-64 \mathrm{~cm}$ & Large timber stand \\
$65-79 \mathrm{~cm}$ & Very large timber stand \\
$=80 \mathrm{~cm}$ & Old trees
\end{tabular}

The first part of the analysis involved grouping trees of similar size. Thus all trees were assigned to diameter classes by applying the age classes in even aged forests provided in Table 15. Maps were depicted showing the spatial arrangement of the trees of the various 
DBH classes and their allotted circular crowns. Using these crown cover maps, the number of trees in each DBH class having crown contact with members of the same DBH class was determined. They constitute a group of trees of similar dimension, the size of which is characterized by the number of group members, ranging from one single tree to a maximum of 36 trees in our investigation. Thus the mean crown radius provided a boundary limit in defining neighbouring and non-neighbouring trees. The frequency of these differently sized groups was then derived. This part of the analysis was computer-aided by using a $\mathrm{C}++$ programed algorithm, which was verified by manual calculations.

Then the Kolmogorov-Smirnoff two sample test (KS test) was conducted to assess the differences between forests. In each diameter class the tree group frequencies were compared with each other. However, the assessment does not include statements of statistically significant differences, because the forests in the study are single populations, not replicated, and no dispersion can be determined. Nevertheless the maximum absolute difference between both distribution functions (d-value) and the p-value of the KS test could be used to indicate certain differences.

\subsubsection{Diameter distribution, diameter differentiation and structural complexity index}

In the second part of the analysis diameter distributions, diameter differentiation (Füldner, 1995) and a structural complexity index were calculated using tree location maps and the measured DBH values. The data from the sample plots were pooled to calculate a diameter distribution specific for each reserve.

The structural complexity index (SCI), developed by Zenner and Hibbs (2000), was calculated with MatLab®. A Delauney-Triangulation was conducted on the basis of the spatial tree distribution, from which a network of non-overlapping triangles with endpoints at the tree stem coordinates was derived. The triangles were raised at their endpoints by an amount corresponding to the height of the trees (estimated by use of regressions of height over DBH in representative samples as mentioned above). The area of the three dimensional triangle constructed was divided by the area of a planar triangle, and the SCI was calculated from the average of the values of the individual triangle pairs produced (in detail in Zenner and Hibbs, 2000). In this way higher SCI values are obtained when greater height differences occur between neighbouring trees. The value 1 , for example, corresponds to a stand with no height difference between the trees. The average SCI value was calculated for both virgin forest reserves from the SCI of all sample plots. 


\subsection{Results}

\subsubsection{Tree groups}

Table 16. Virgin forest Kyjov: Frequency of tree groups on 10 ha. The trees of each group belong to one DBH class. Criterion for grouping is crown contact.

\begin{tabular}{|c|c|c|c|c|c|c|c|}
\hline \multirow{2}{*}{$\begin{array}{c}\text { Number of } \\
\text { trees } \\
\text { per group }\end{array}$} & \multicolumn{7}{|c|}{$\mathrm{dbh}$ classes $[\mathrm{cm}]$} \\
\hline & $7-15$ & $16-20$ & $21-34$ & $35-49$ & $50-64$ & $65-79$ & 80 \\
\hline 1 & 299.4 & 252.0 & 183.0 & 111.0 & 82.7 & 72.0 & 53.6 \\
\hline 2 & 108.0 & 59.0 & 54.4 & 33.7 & 27.6 & 20.7 & 12.3 \\
\hline 3 & 60.5 & 16.1 & 29.1 & 23.7 & 14.6 & 13.8 & 2.3 \\
\hline 4 & 22.2 & 6.9 & 11.5 & 8.4 & 5.4 & 3.1 & 2.3 \\
\hline 5 & 24.5 & 2.3 & 3.1 & 5.4 & 5.4 & 3.8 & \\
\hline 6 & 8.4 & 0.8 & 5.4 & 2.3 & 3.8 & 1.5 & \\
\hline 7 & 7.7 & & 2.3 & 1.5 & 3.1 & & \\
\hline 8 & 9.2 & & 2.3 & 1.5 & 1.5 & & \\
\hline 9 & 3.1 & & & & 0.8 & 0.8 & \\
\hline 10 & 6.1 & & 2.3 & 0.8 & & & \\
\hline 11 & 3.1 & & & 0.8 & & & \\
\hline 12 & 2.3 & & 0.8 & & & & \\
\hline 13 & 0.8 & & & 0.8 & & & \\
\hline 14 & & & & & & 0.8 & \\
\hline 15 & 0.8 & & 0.8 & & & & \\
\hline 16 & 1.5 & & 0.8 & & & & \\
\hline 17 & 0.8 & & 0.8 & & & 0.8 & \\
\hline 18 & & & & & & & \\
\hline 19 & & & & & & & \\
\hline 20 & & & 0.8 & & & & \\
\hline 21 & 0.8 & & & & & & \\
\hline 22 & 0.8 & & & & & & \\
\hline 23 & & & & & & & \\
\hline 24 & 1.5 & & & & & & \\
\hline 25 & & & & & & & \\
\hline 26 & 0.8 & & & & & & \\
\hline 27 & & & & & & & \\
\hline 28 & 0.8 & & & & & & \\
\hline 29 & & & & & & & \\
\hline 30 & & & & & & & \\
\hline 34 & 0.8 & & & & & & \\
\hline 36 & 0.8 & & & & & & \\
\hline percentage & & & & & & & \\
\hline $\begin{array}{l}\text { of trees } \\
\text { per dbh class }\end{array}$ & 41.5 & 13.1 & 16.8 & 10.6 & 8.8 & 6.4 & 2.7 \\
\hline
\end{tabular}


Table 17. Selection forest UA1: Frequency of tree groups on 10 ha. The trees of each group belong to one DBH class. Criterion for grouping is crown contact.

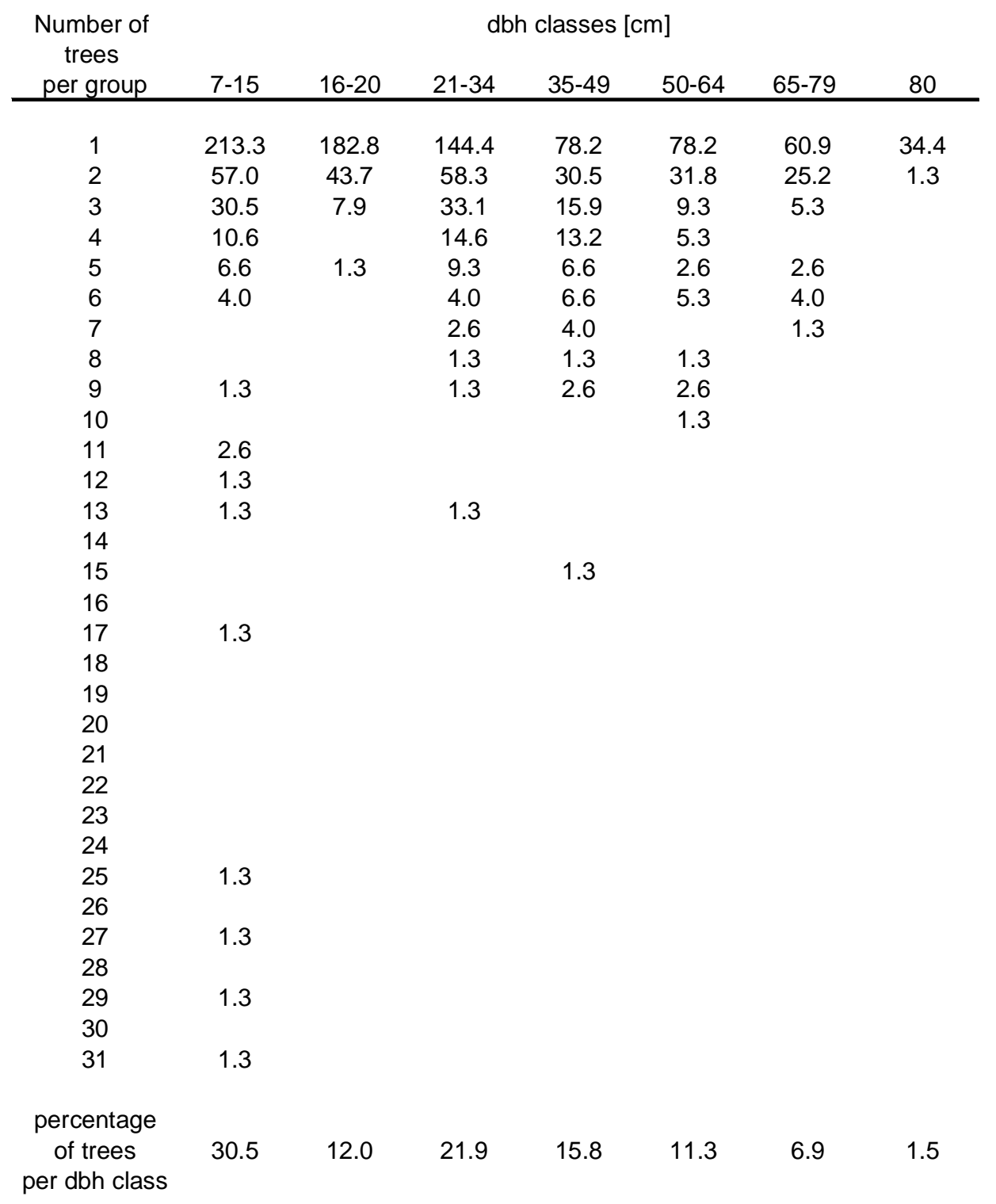


Table 18. Stand Y166: Frequency of tree groups on 10 ha. The trees of each group belong to one DBH class. Criterion for grouping is crown contact.

\begin{tabular}{|c|c|c|c|c|c|c|c|}
\hline \multirow{2}{*}{$\begin{array}{l}\text { Number of } \\
\text { trees } \\
\text { per group }\end{array}$} & \multicolumn{7}{|c|}{$\mathrm{dbh}$ classes $[\mathrm{cm}]$} \\
\hline & $7-15$ & $16-20$ & $21-34$ & $35-49$ & $50-64$ & $65-79$ & 80 \\
\hline 1 & & & 27.59 & 111.72 & 59.31 & 57.93 & 2.76 \\
\hline 2 & & & 2.76 & 27.59 & 37.24 & 5.52 & \\
\hline 3 & & & & 17.93 & 20.69 & & \\
\hline 4 & & & & 9.66 & 11.03 & & \\
\hline 5 & & & & 1.38 & 5.52 & & \\
\hline 6 & & & & 1.38 & 5.52 & & \\
\hline 7 & & & & 1.38 & 4.14 & & \\
\hline 8 & & & & & 4.14 & & \\
\hline 9 & & & & & 5.52 & & \\
\hline 10 & & & & 2.76 & 1.38 & & \\
\hline 11 & & & & & 1.38 & & \\
\hline 12 & & & & & & & \\
\hline 13 & & & & & 1.38 & & \\
\hline 14 & & & & & 2.76 & & \\
\hline 15 & & & & & & & \\
\hline 16 & & & & & & & \\
\hline 17 & & & & & & & \\
\hline 18 & & & & & & & \\
\hline 19 & & & & & & & \\
\hline 20 & & & & & & & \\
\hline percentage & & & & & & & \\
\hline $\begin{array}{l}\text { of trees } \\
\text { per dbh class }\end{array}$ & & & 3.62 & 34.09 & 54.45 & 7.54 & 0.30 \\
\hline
\end{tabular}

In each stand the number of tree groups decreased exponentially as the tree number per group increased. But the stands differed in the steepness of decline as well as in number of groups per dbh class. Table 16-18 give numbers of tree groups projected on an area of 10 ha. On this area 1.721 groups were found in Kyjov (Table 16). $41.5 \%$ of trees belonged to the lowest DBH class 7-15 cm. In this class 299 single trees and 108 two-tree groups occured. The largest group comprised of 36 trees. $30 \%$ of trees form single tree groups and $14 \%$ form groups with 10 or more trees. $4.2 \%$ of trees form groups with 10 or more individuals and have a DBH of more than $20 \mathrm{~cm}$. In Havešová a very similar distribution of tree groups was determined. 
Similar results were also seen in the selection forest UA1 (Table 17). The greatest difference to virgin forests was a narrower diameter range. In detail, the DBH class $65-79 \mathrm{~cm}$ demonstrated less large tree groups than in virgin forests. In total 1.275 groups were determined. Stand UA2 demonstrated similar tree groups as UA1.

Clear differences were seen when comparing the virgin forests and the even aged forest stands. Table 18 gives the results exemplarily for Y166. 530 tree groups were determined on an area of 10 ha. When comparing our findings in the virgin and selection stands the largest groups belonged to the $50-59 \mathrm{~cm} \mathrm{DBH}$ class. This class also comprised of the most trees, with lower tree numbers in adjacent upper and lower DBH classes. The number of groups also decreased with increasing size. However, in the 50-59 $\mathrm{cm}$ DBH class the decline down to the 10 tree group was not as steep as in the virgin and selection forests.

Table 19 shows KS test results of compared DBH class distributions. Clear differences between uneven aged and even aged forests were found. In unevenaged selection forests, the frequencies of tree groups was similar to that in virgin forests. The d-value stated the smallest absolute differences in each diameter class between the virgin and the selection forest. $\mathrm{d}-$ and p-values of the KS test revealed differences in the lowest DBH class only. Absolute differences were high, if uneven aged forests were compared with the even aged one. The pvalue stated differences in class $7-15 \mathrm{~cm}$ between the virgin and the selection forest. The selection forest and the even aged forest differed in each DBH class except class $\geq 80 \mathrm{~cm}$. The virgin and even aged forest differed from 7 to $34 \mathrm{~cm}$ and from 50 to $79 \mathrm{~cm}$.

Table 19. Differences between tree group distributions in table 3-5 using the KolmogorovSmirnoff two sample test (missing values in classes, where no trees occured in Stand Y166)

\begin{tabular}{ccc} 
pair of compared distributions & $\mathrm{d}$ & $\mathrm{p}$ \\
\hline \hline Kyjov - UA1 $(7-15 \mathrm{~cm})$ & 0.11 & 0.001 \\
Kyjov - UA1 $(16-20 \mathrm{~cm})$ & 0.04 & 0.984 \\
Kyjov - UA1 $(21-34 \mathrm{~cm})$ & 0.08 & 0.287 \\
Kyjov - UA1 $(35-49 \mathrm{~cm})$ & 0.11 & 0.244 \\
Kyjov - UA1 $(50-64 \mathrm{~cm})$ & 0.04 & 1.000 \\
Kyjov - UA1 $(65-79 \mathrm{~cm})$ & 0.09 & 0.806 \\
Kyjov - UA1 (more or equal & 0.20 & 0.308 \\
than $80 \mathrm{~cm})$ & & \\
& & \\
Kyjov - Y166 $(7-15 \mathrm{~cm})$ & - & - \\
Kyjov - Y166 $(16-20 \mathrm{~cm})$ & - & - \\
Kyjov - Y166 $(21-34 \mathrm{~cm})$ & 0.28 & 0.021 \\
Kyjov - Y166 $(35-49 \mathrm{~cm})$ & 0.06 & 0.869 \\
Kyjov - Y166 $(50-64 \mathrm{~cm})$ & 0.20 & 0.005 \\
Kyjov - Y166 $(65-79 \mathrm{~cm})$ & 0.30 & 0.001 \\
Kyjov - Y166 (more or equal & 0.23 & 0.998 \\
than $80 \mathrm{~cm})$ & & \\
& & \\
UA1 - Y166 $(7-15 \mathrm{~cm})$ & - & - \\
UA1 - Y166 $(16-20 \mathrm{~cm})$ & - & - \\
UA1 - Y166 $(21-34 \mathrm{~cm})$ & 0.37 & 0.001 \\
UA1 - Y166 $(35-49 \mathrm{~cm})$ & 0.15 & 0.048 \\
UA1 - Y166 $(50-64 \mathrm{~cm})$ & 0.20 & 0.005 \\
UA1 - Y166 $(65-79 \mathrm{~cm})$ & 0.29 & 0.003 \\
UA1 - Y166 (more or equal & 0.03 & 1.000 \\
than $80 \mathrm{~cm})$ & &
\end{tabular}



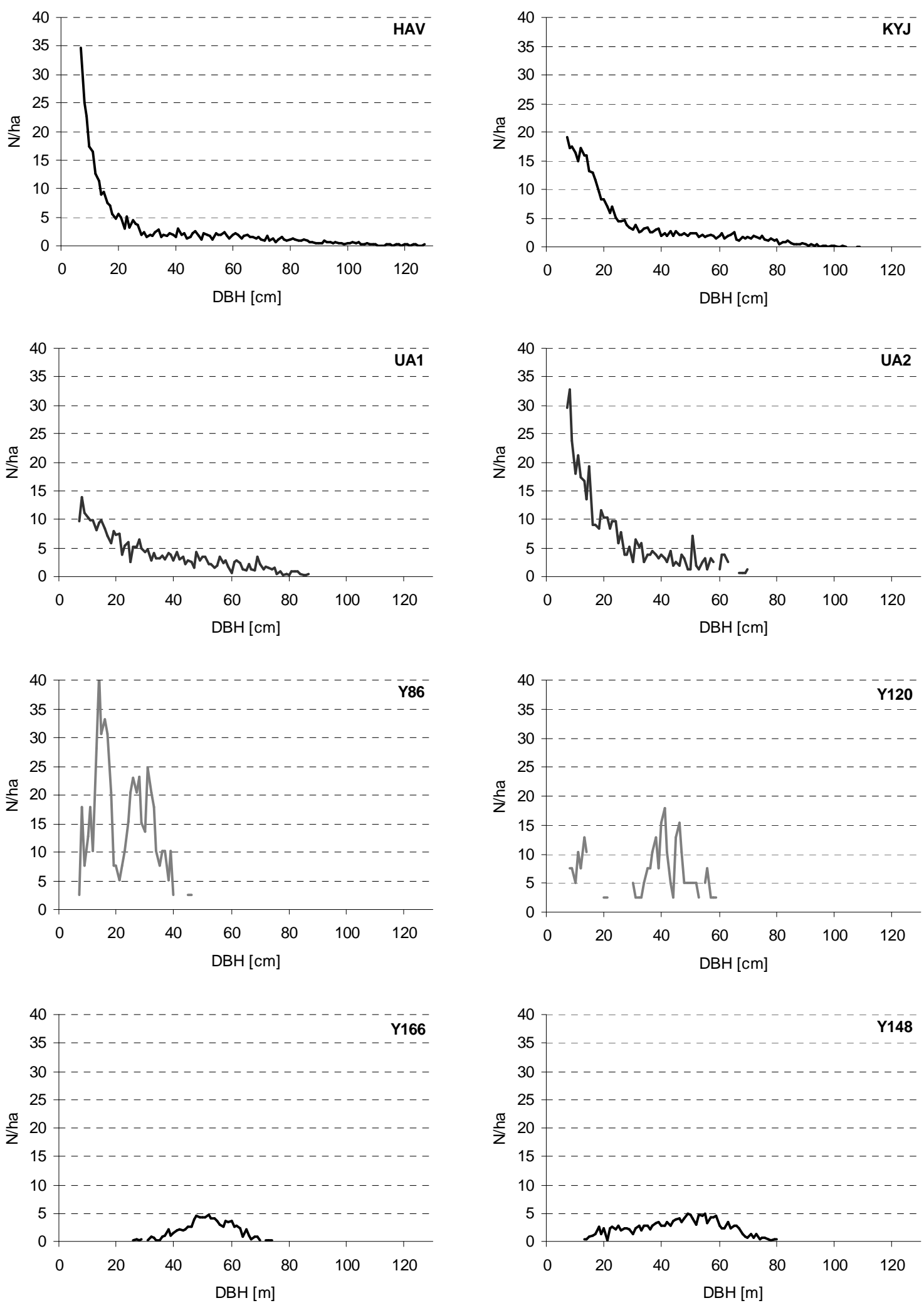

Fig. 14. Diameter distributions of the investigated stands (The small data set for Y86 and Y120 limits interpretation to broad trends.) 


\subsubsection{Diameter distribution}

The diameter distributions showed a wider range in virgin than in managed forests (Fig. 14). In uneven aged forests the number of trees decreased with increasing DBH. While this decline could be fitted by the function, $\mathrm{N}=\mathrm{A} \times \exp ^{(-\mathrm{B} \times \mathrm{DBH})}$ for Havešová, Kyjov and UA2 $\left(\mathrm{R}^{2} \sim 0.8\right)$, it was less successful for UA1 due to the smaller decline in tree numbers around DBH $10 \mathrm{~cm}$. However, Kyjov also differed from Havešová by having fewer of the smallest trees and more trees between $12-30 \mathrm{~cm}$.

The even aged forests Y86 and Y120 had a two peak distribution with a first peak at $15 \mathrm{~cm}$ DBH and the second at 30 and $40 \mathrm{~cm}$ respectively. In older stands, a bell curve was apparent with a maximum tree number at $50 \mathrm{~cm} \mathrm{DBH}$.

\subsubsection{Diameter differentiation}

Füldner's (1995) index of diameter differentiation describes size differences in relation to the nearest neighbour (Fig. 15). T1(0.7-1) shows the percentage of tree pairs where the difference in diameter of a tree pair ranges from 70 to $100 \%$. In Havešová one third of the trees had diameters differing from the nearest neighbour by at least $70 \%$. Conversely, in Y166 the diameters of $85 \%$ of trees differed from the nearest neighbour by less than $30 \%$.

The mean diameter differentiation in the virgin forest Havešová is $5 \%$ higher than in the selection stand UA1. But stand UA2 has a higher differentiation than the virgin forest Kyjov. The differentiation in the even aged stands Y86 and Y120 was only 3-8\% smaller than in uneven aged stands. The diameter differentiation in Y166 was $27 \%$ smaller.

In virgin forests the individual values for the 0.4 ha plots ranged between 17 and $41 \%$ for T1(0.7-1), 7 and $27 \%$ for T1(0.5-0.7), 11 and $32 \%$ for T1(0.3-0.5), and 19 and $50 \%$ for $\mathrm{T} 1(0-0.3)$. When the index was calculated for the second and third closest neighbour, the Tvalues were similar. 


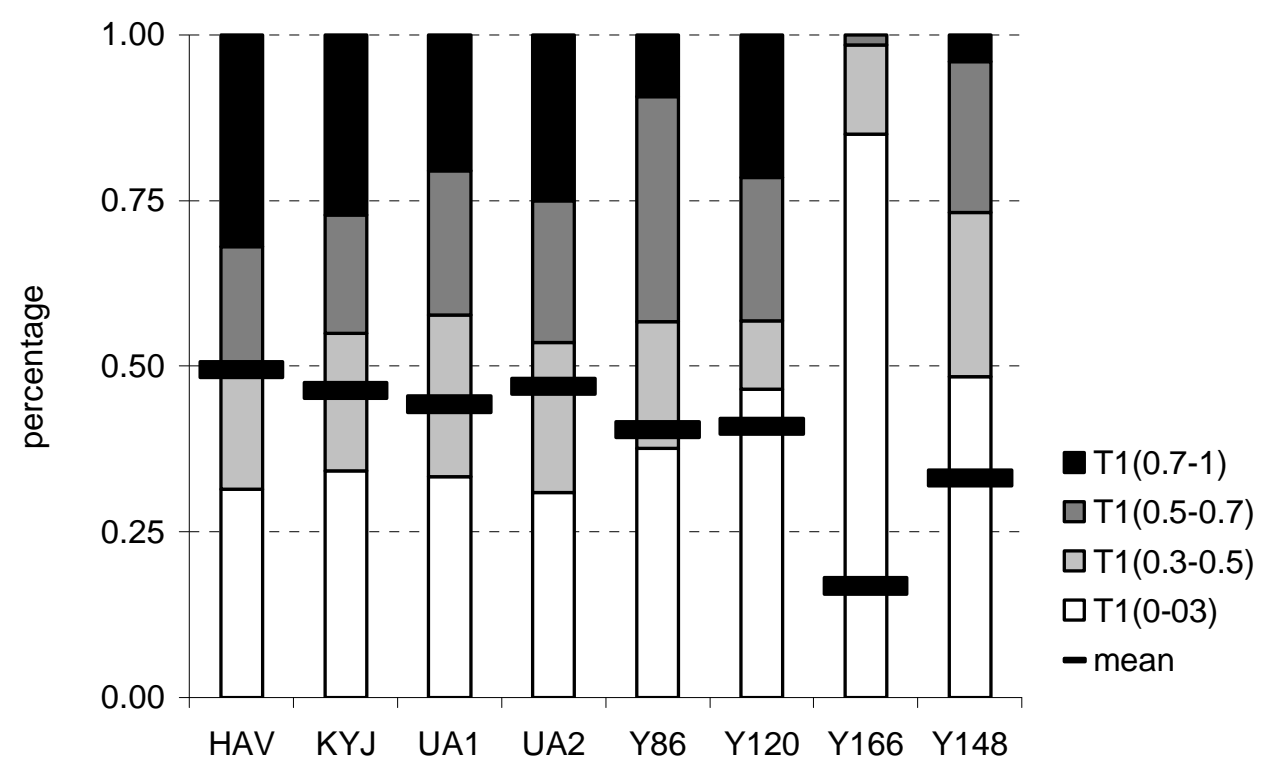

Fig. 15. Füldner's (1995) diameter differentiation between nearest neighbours in two virgin forests and six managed forests

\subsubsection{Structural complexity index}

The SCI revealed the highest heterogeneity for the virgin forest Havešová (table 20), where the plot values ranged from 3.06 to 4.96. The values for the second virgin forest Kyjov were similar to those of UA1 and UA2, ranging from 1.98 to 2.83. The values for Y166 and Y148 were lower than for unevenaged stands. However, the values for Y86 and Y120 approximated (surprisingly) the values for virgin forests.

Table 20. Structural Complexity Index (SCI) from Zenner and Hibbs (2000) in two virgin forests and six managed forests.

\begin{tabular}{ll} 
Stand & SCI \\
\hline HAV & 3.89 \\
KYJ & 2.48 \\
UA1 & 2.11 \\
UA2 & 2.56 \\
Y86 & 3.01 \\
Y120 & 3.09 \\
Y166 & 1.40 \\
Y148 & 1.03
\end{tabular}




\subsection{Discussion}

\subsubsection{Tree groups}

In all eight beech forests, groups composed of only single trees dominated over groups that had many similarly sized trees. Our hypothesis could not been refuted, that mixes of single trees of different sizes dominate in virgin forests and that the number of groups of similarly sized trees decreases exponentially as group size increases. This rule was also observed in the managed stands however, they differed in decline. Thus, baseline information concerning frequencies of differently sized tree groups facilitates a quantitative comparison of forests with different structures.

Gap studies in virgin forests suggest that single tree harvesting in conjunction with group selection harvesting best imitates the natural disturbance regime in beech forests (Drößler and Lüpke, 2005). Single tree harvesting prevails also in selection forests with a less proportion of group selection harvesting. However the similarity of group size frequencies in virgin and selection forests is similar.

An interesting result in virgin forests is that the number of tree groups and the number of gaps decreased exponentially with increasing size. This result was also valid for several DBH class divisions and enlarged radii to define neighbours. Thus a mean area of development stages is no adequate value to describe forest development.

To distinguish between species dependent pattern of tree groups and random patterns a random tree distribution could be simulated with the aid of a computer. After a comparison with the real group patterns, statements concerning more clustered or seperated growing of trees can be made.

For us the frequencies of differently sized tree groups seem to be instructive, because they deliver a picture of stand structure, which is relevant to silvicultural operations. Contrary to diameter differentiation (Füldner, 1995) the grouping results in absolute values per stand and trees are the basal unit. Therefore foresters can simulate tree group distributions in the forest. By knowing real tree arrangements the forester is able to estimate the effort required to achieve a certain stand structure, because it is this kind of thinking he is used to in thinning operations. For example, in uneven aged forests a comparison with natural structures in virgin forests can be drawn.

Criticism may be directed at the selection of DBH classes, which influences the frequency of groups. We chose class widths according to age classes in managed forests to achieve a good 
comparability with these forests. A grouping with other class widths was also in accordance with our hypothesis of an exponential decline of groups with increasing size (= number of group members).

The algorithm can be enlarged to calculate additional parameters as tree density per group or class, presence of individual trees from other diameter classes, or area covered by the group. The latter would provide the proportion of forest development stages. Grassi et al. (2003) calculated the area of development stages in a comparable manner.

Depending on the purpose of an investigation the structural indicators other than forest development phases and tree grouping may also be used to describe forest structure. The indicators described by Pommerening (2002) and Zenner and Hibbs (2000) can also be used to distinguish structurally different close-to-nature forests:

\subsubsection{Diameter distribution and diameter differentiation}

The diameter distributions allow an assessment of the degree of "naturalness" of a forest structure with relatively little effort. A reliable assessment can be made from the diameter range and the position of the maximum for stands (see Figure 14). The number and location of maxima can also reflect different forest structures in even aged forests (Burschel and Huss, 1997). In selection forests, derivations from an exponential distribution recommend the removal or promotion of trees in a certain DBH class as shown by Schütz (2001).

However, different diameter distributions between virgin forests are shown by Westphal et al. (2006). One correct diameter distribution for all natural forests does not exist. It is even not proven, whether typical distributions are unequivocally exponential or may have a more constant decline of tree number after a steep decline. The distributions found in Havešová and Kyjov demonstrate for trees larger than $30 \mathrm{~cm} \mathrm{DBH}$ a very low mortality.

By diameter differentiations the local mixing of different sized trees could be assessed and a reasonable ranking between the forests was possible. As Füldner (1995) stated this indicator is most useful to assess the differentiation before and after thinnings. However, compared with diameter distributions no operational guidance can be given. 


\subsubsection{Structural complexity index}

The Structural Complexity Index (Zenner and Hibbs, 2000) allowed one to differentiate diverse heterogeneous structures. By definition, the SCI is influenced by height and the distance between trees. Therefore stands of different ages cannot be compared. Zenner and Hibbs (2000) investigated douglas fir stands between 80 and 300 years old (maximum height 50-70 m, stand density 294-350 trees per ha). They found that SCI was largely influenced by vertical parameters and less by the spatial distribution of trees. In their study, SCI was found to be dependent on the number of particularly large trees and the standard deviation in tree height.

In our study, the high structural complexity in Stand Y86 and Y120 was caused by understorey trees being equally distributed throughout the plots. The surprisingly low SCI value in Stand Y148 was caused by a very dense upperstorey and a high mortality of smaller trees in the last decades. In accordance to the SCI values the structural complexity (dimension and position of live trees) in virgin forests and selection forests was similar. Higher SCI values in Havešová we explain by taller trees.

Zenner and Hibbs (2000) recommended the SCI for describing different forest patches quantitatively. However, forest patches differ in tree height and density. Besides structural complexity these differences effect the SCI also.

Other indicators to describe tree distribution pattern as Clark-Evans-Index (Clark and Evans, 1954), pair correlation and mark correlation functions (Stoyan and Stoyan, 1994) are presented in section 6. They did not allow silviculturally helpful descriptions of forest structure, but relative rankings between forests could derived.

\subsection{Conclusions}

There are different ways to quantify forest structure. Depending on the research aims there are numerous indicators available. The first step is to clarify the research scale i.e., stand or individual tree level. Concerning stand level, diameter distributions work well for assessments of stand development and have great practical application because they balance information gained and effort required. Diameter differentiation and complexity index do not support silvicultural decisions. They rank forest structure and have mere research applications.

Concerning individual tree level, the grouping of similar sized trees provides practical guidance for transforming a forest to a specific structure. For example, the frequency distribution of differently sized tree groups in virgin forests can provide information about 
tree removals required to achieve more natural structures. But the grouping and its quantification is applicable independently from silviculturally aimed forest structure.

\subsection{References}

Burschel, P., Huss, J. 1997. Grundriß des Waldbaus. Parey. Berlin. German.

Clark, P. J., Evans, F. C. 1954. Distance to nearest neighbour as a measure of spatial relationships in populations. Ecology 35: 445-453.

Drößler, L. 2001. Untersuchungen zum Aufbau eines plenterartigen Laubholzbestandes mit hohem Edellaubholzanteil. Master thesis Univ. Göttingen. German (not published).

Drößler, L., Lüpke, B. v. 2005. Canopy gaps in two virgin beech forest reserves in Slovakia. Journal of Forest Science 51: 446-457.

Drößler, L., Lüpke, B. v. 2006. Verjüngung, Wachstumsfaktoren und Holzvorrat in zwei slowakischen Buchenurwald-Reservaten. AFJZ (in press). German.

Füldner, K. 1995. Strukturbeschreibung von Buchen-Edellaubholz-Mischwäldern. PhD. thesis Univ. Göttingen. Cuvillier. Göttingen. German.

Grassi, G., Minotta, G., Giannini, R., Bagnaresi, U. 2003. The structural dynamics of managed uneven-aged conifer stands in the Italian eastern Alps. Forest Ecology and Management 185: 225-237.

Kahle, M., Stüber, V., Pommerening, A. 1996. Strukturbeschreibung in Buchen-Edellaubholzbeispielbeständen. Abschlussbericht. Inst. f. Forsteinrichtung. Univ. Göttingen.

Kern, K. G. 1966. Wachstum und Umweltfaktoren im Schlag- und Plenterwald. Schriftenr. forstl. Abt. Univ. Freiburg i. Br. 5. German.

Korpel', Š. 1995. Die Urwälder der Westkarpaten. Gustav Fischer. Stuttgart, Jena, New York. German. Meyer, P. 1999. Bestimmung der Waldentwicklungsphasen und der Texturdiversität in Naturwäldern. AFJZ 170: 203-211. German.

Pommerening, A. 2002. Approaches to quantifying forest structures. Forestry, Vol. 75: 305-324.

Schrempf, W. 1986. Waldbauliche Untersuchungen im Fichten-Tannen-Buchen-Urwald Rothwald und in Urwald-Folgebeständen. Verbandwiss. Ges. Österr. Wien. German.

Schütz, J.-P. 2001. Der Plenterwald und weitere Formen strukturierter und gemischter Wälder. Parey. Berlin. German.

Stoyan, D., Stoyan, H. 1994. Fractals, Random Shapes and Point Fields. Methods of Geometrical Statistics. J. Wiley. Chichester, New York, Brisbane, Toronto, Singapore.

Tabaku, V. 1999. Struktur von Buchen-Urwäldern in Albanien im Vergleich mit deutschen BuchenNaturwaldreservaten und -Wirtschaftswäldern. PhD. thesis Univ. Göttingen. Cuvillier. German.

Westphal, C., Tremer, N., Oheimb, G.v., Hansen, J., Gadow, K.v., Härdtle, W. 2006. Is the reverse J-shaped diameter distribution universally applicable in European virgin beech forests? For. Ecol. Manage. 223, 75-83. Winter, S., Flade, M., Schumacher, H., Möller, G. 2003. Nutzungsstandards für die Bewirtschaftung von Buchenwäldern im norddeutschen Tiefland. Sachbericht der Landesanstalt für Grossschutzgebiete (Brandenburg). F+E-Vorhaben Biologische Vielfalt und Forstwirtschaft. German.

Zenner, E. K., Hibbs, D. E. 2000. A new method for modeling the heterogeneity of forest structure. Forest Ecology and Management 129: 75-87. 


\section{Canopy gaps}

Dieser Teil entspricht einer Publikation von L. Drößler und B. v. Lüpke im Jahr 2005:

Canopy gaps in two virgin beech forest reserves in Slovakia. Journal of Forest Science 51: 446-457.

\subsection{Abstract}

The formation, size distribution and percentage of gaps in virgin forests are presented in two case studies. Gaps are defined as openings in the canopy above 2/3 stand height. These gaps comprise $16 \%$ of the forest area in Havešová Reserve and $15 \%$ of the forest area in Kyjov Reserve. The estimated turnover time is 220 years.

More than half the gaps were caused by the death of one tree, and $80 \%$ of the gaps were due to the death of up to 3 trees. The largest gap in Havešová Reserve was 0.40 ha, resulting from the death of 56 trees over the last 40 years. The area of the largest gap in Kyjov Reserve was 0.44 ha. It was caused by the death of 80 trees over the last 40 years. Such large gaps are rare. $85 \%$ of the gaps are smaller than $250 \mathrm{~m}^{2}$.

A correction of the bias towards the over-representation of large gaps by line-transect sampling was made by estimating the percentage area of different sized gaps from the percentage length along the transects.

In Havešová an attempt was made to date the death of trees that were still visible in the gaps. $1 / 5$ of the trees initiated gaps by their death, while $4 / 5$ of the trees extended gaps. While uprooted trees dominate in Havešová, breakage is most common in Kyjov.

Keywords: natural disturbance, gap size distribution, gap formation, European beech

\subsection{Introduction}

The creation of gaps in harvesting operations offers a good opportunity to form forests closer to nature. If naturalness is a management aim the percentage of gaps in the forest area, gap size distributions and gap formation rates should be known. Yet investigations of gap size in virgin European beech forests are rare. Tabaku and Meyer (1999) describe gap patterns in three Albanian forest stands, and Zeibig et al. (2004) describe a forest in Slovenia. Additional research undertaken to enhance knowledge about the nature of gap patterns in relation to site and aspect would make a useful contribution to the existing worldwide investigations of gaps in beech forests (e.g. Runkle, 1982; Nakashizuka, 1988; Yamamoto and Nishimura, 1999). By 
way of contribution this paper presents two case studies in which gap investigations were undertaken in virgin beech forests.

\subsection{Material}

The research sites are located in eastern Slovakia. The Havešová Reserve is situated at 500$650 \mathrm{~m}$ asl. on a south facing slope in the Beskids Mountains and covers an area of 170 ha. The Reserve comprises mainly the plant association Dentario glandulosae - Fagetum with a gradient to Carici pilosae - Fagetum on the upper slope. The annual rainfall ranges from 700$800 \mathrm{~mm}$ (450 $\mathrm{mm}$ in the growing season) and the average annual temperature is $7^{\circ} \mathrm{C}$. A welldeveloped mesotrophic brown earth covers sedimentary sandstone, occasionally interspersed with shale. The moderately acidic soil has good water holding capacity and nitrogen availability. The stand height is approximately $45 \mathrm{~m}$, but isolated trees grow higher than $50 \mathrm{~m}$. The Kyjov Reserve is situated at 700-820 $\mathrm{m}$ asl. on a north facing slope in the Vyhorlat Mountains and covers an area of 53 ha. It comprises the plant association Dentario glandulosae - Fagetum. The annual rainfall ranges from 750-800 $\mathrm{mm}$ and the average annual temperature is $6{ }^{\circ} \mathrm{C}$. The mesotrophic brown earth over andesite is moderately acidic and has good water holding capacity and nutrient availability. Stand height is approximately $30 \mathrm{~m}$, although the largest trees can reach $34 \mathrm{~m}$. At Kyjov tree height growth is limited by the occurrence of shallow soils, exposure of sites to strong winds and, sometimes, icy slopes due to the presence of an artificial lake nearby.

A subcontinental climate prevails in both Reserves, which are located $50 \mathrm{~km}$ apart. The mixture of other tree species (Acer pseudoplatanus, A. platanoides, Fraxinus excelsior, Ulmus glabra) is less than $1 \%$. A more extensive description of the forest stand structure is given in Korpel (1995).

\subsection{Methods}

\subsubsection{Gap definition}

In each Reserve the DBH and height of 60 trees, regularly distributed in all size-classes, were measured to obtain stand height curves. These curves facilitated the estimation of stand height and the division of stands into 3 height strata. Following Runkle's (1992) recommendations, a gap was recorded where a canopy opening occurred in the upper stratum above $2 / 3$ of dominant tree height. The stand height at Havešová was $45 \mathrm{~m}$, with the upper stand stratum beginning at $30 \mathrm{~m}$ height. The stand height in Kyjov was $30 \mathrm{~m}$, with the upper stratum beginning at $20 \mathrm{~m}$ height. 

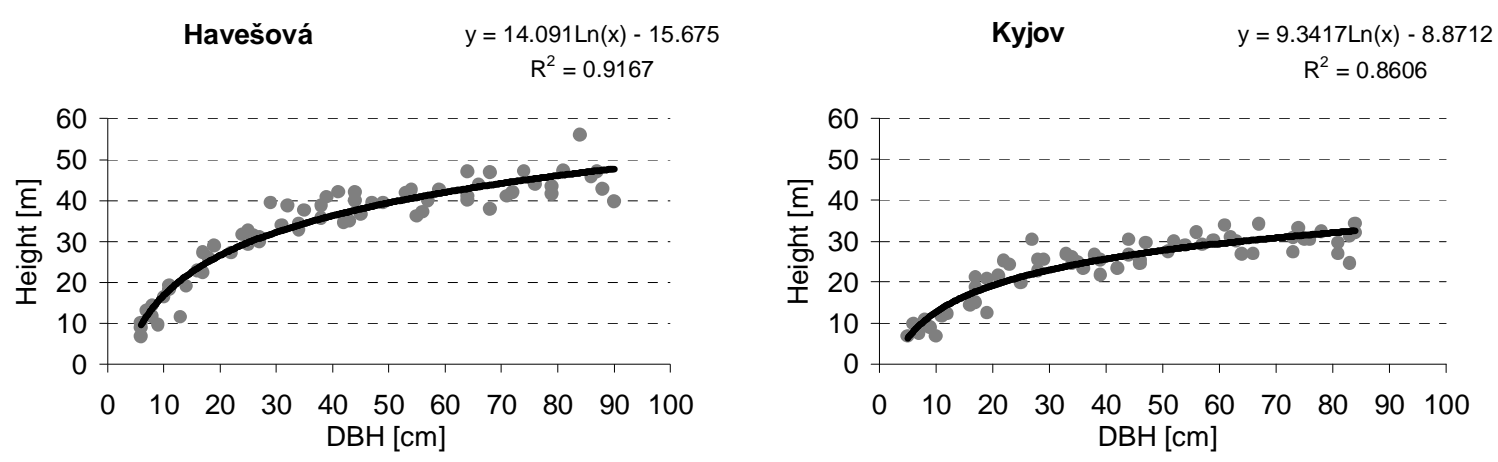

Fig. 16. Stand height curves for Havešová and Kyjov Reserves

According to the height curves, a tree growing into the upper stratum has a DBH of approximately $30 \mathrm{~cm} \mathrm{DBH}$ at Havešová and $20 \mathrm{~cm} \mathrm{DBH}$ at Kyjov. These DBH values were used to distinguish between gaps with regeneration and closed forest.

Gaps along the transects, with the boundary between gap regeneration and closed forest defined by $7 \mathrm{~cm} \mathrm{DBH}$, were also measured to facilitate comparisons with other gap investigations.

In accordance with Runkle (1992) gaps were not confined to the area directly below the canopy opening. The extended gap, that is the area between the bases of the trees forming the gap boundary (Figure 17), was also recorded.

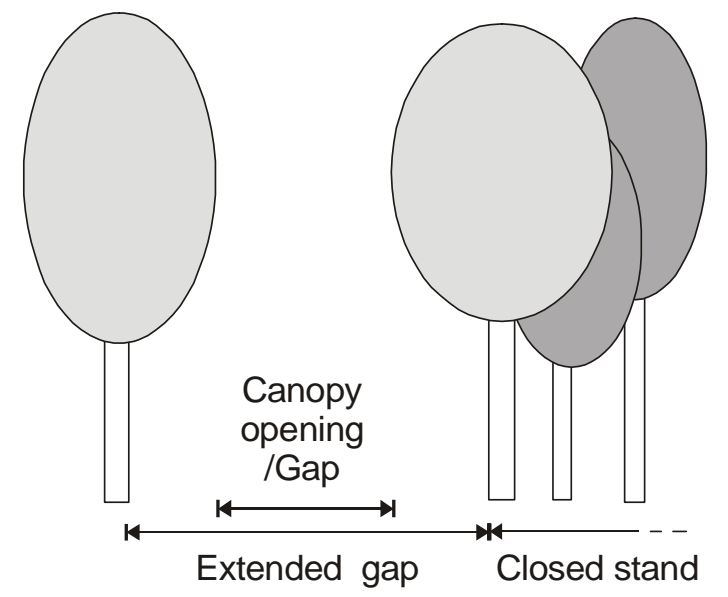

Fig. 17. Gap definitions for canopy opening and extended gap 


\subsubsection{Gap sampling}

A grid of line transects, similarly apportioned parallel and perpendicular to the slope, were used to determine the percentage area of gaps in the forests. The segments along the transects were measured where canopy openings, extended gaps, and closed forest cover occurred. The percentage length of these forest characteristics along the transects corresponded to the percentage area in the forest.

Atypical sites, such as deeply eroded streambeds or sites with slopes $>30^{\circ}$, were excluded from further measurements. Eight gaps in Havešová were thus excluded. For the remaining gaps the area of the canopy gap and extended gap were measured. Adopting Runkle's (1992) recommendation the formula for calculating an ellipse was used, where the maximum width of the gap and the width at right angles to this length constituted the length and width of the ellipse respectively. Where more than 10 dead canopy trees occurred in a gap, or in other incidences where gap shapes were non-elliptical, gap area was derived as a polygon. Dead canopy trees with $\mathrm{DBH} \geq 30 \mathrm{~cm}$ in Havešová and $\mathrm{DBH} \geq 20 \mathrm{~cm}$ in Kyjov were recorded in the extended gap and regarded as gap creating trees. The nature of death (uprooting, partial uprooting, breakage with stump height, standing death and partial death) was recorded in accordance with Runkle (1992).

\subsubsection{Representative percentage of different sized gaps}

When sampling with line transects, large gaps are over-represented. Since the relative probability that a gap is crossed by the transect line is equal to the gap diameter Runkle (1984) takes the square root of the area of every gap, which is proportional to the diameter of a circular gap. However, one may also correct the bias by estimating the percentage gap area from the percentage gap length along the transects. This error of estimation needs to be calculated for each gap. The first step is to calculate the percentage gap length from the total transect length. Since this percentage gap length corresponds to the percentage gap area in the research area, percentage gap area can be estimated. The second step is to calculate the percentage area of each gap from the total area of all gaps. The percentage length of a gap would correspond to the percentage area if the gap has been sampled representatively. Differences may be corrected by the quotient derived from the percentage length of a gap along the transect length and the percentage area of this gap. This quotient represents one gap. The theoretical number of gaps is then obtained from the sum of the quotients of all gaps. The representative percentage of each gap is derived from the theoretical number of gaps. 
Example: Assume there is a single-tree gap with the length $8.2 \mathrm{~m}$ along the transects, comprising an area of $42 \mathrm{~m}^{2}$. All gaps have a total length of $815.1 \mathrm{~m}$ along the transect with a total area $28288 \mathrm{~m}^{2}$. Thus the percentage length of the single-tree gap along the entire transect is $1.006 \%$, and yet the percentage of the total gap area is only $0.148 \%$. The ratio of these factors provides a correction factor: $1.006 / 0.148=6.797$. By adding up the correction factors of each gap we calculate the theoretical number of gaps to be 318.6. Consequently the representative percentage of the single-tree gap is $2.13 \%(=6.797 / 318.6)$.

To obtain representative percentages of dead trees (Figure 8, 10, Tables 3, 4), every dead tree was given the representative value of its gap. Theoretical absolute frequencies were then converted to relative frequencies.

\subsubsection{Estimating the age of dead trees}

In Havešová an attempt was made, to estimate the year of death of gap creating trees. Initial estimates were based on the observed state of decay. However sometimes the actual year in which trees, estimated to be in a similar state of decay, died differed by 10 years. In most cases trees in the understorey, or regeneration demonstrated rapid acceleration of shoot growth or damage as a result of release. Such events could be dated by counting the annual bud scars and provided accurate estimates of the year of death for trees that had died in the previous 20 years. For trees that had died earlier, a core was extracted from trees released as a result of tree death to identify sudden increases in annual ring width. The error in the dating is subjectively estimated to be $+/-5$ years for periods longer than 20 years and $+/-10$ years for periods longer than 30 years.

In Kyjov the estimation of the year of death was limited to trees that died in the previous 10 years. Estimates were difficult, as vigorous ground vegetation had impeded regeneration growth in the intervening years. In Havešová the seedlings were not impeded by such vigorous undergrowth following gap creation and were already partly visible.

\subsubsection{Gap formation rate}

Runkle (1992) calculates gap formation rate as the percentage of total gap area $\leq \mathrm{n}$ years old divided by $\mathrm{n}$. In this study the age of dead trees and not gap age was recorded because usually trees in one gap had not died in the same year. Thus the calculation of gap formation rate is not based on the proportion of gaps formed during a certain time period, but on the proportion of trees that died in that period. The proportion of dead trees < n years was multiplied by gap percentages. This reduced value was divided by $\mathrm{n}$ to obtain an annual gap formation rate. 
Example: The gap percentage area in Kyjov is $14.63 \% .31 .2 \%$ of dead trees in Kyjov had died in the last 10 years. Thus the annual rate of gap formation is $0.456 \%(14.63 \%$ x $0.312=$ $4.56 \%$ ). The reciprocal value of the gap formation rate provides turnover time (Runkle, 1992), which, in this example, is 219 years.

\subsection{Results}

\subsubsection{Gap percentages}

Tab. 21. Percentage area of canopy gaps in the forest area: gap closure by regrowth reaching $2 / 3$ of the stand height (see 3.1.)

\begin{tabular}{lcccc}
\hline & $\begin{array}{c}\text { total transect } \\
\text { length }\end{array}$ & $\begin{array}{c}\text { canopy } \\
\text { gaps [\%] }\end{array}$ & $\begin{array}{c}\text { extended } \\
\text { gaps [\%] }\end{array}$ & $\begin{array}{c}\text { closed } \\
\text { stand [\%] }\end{array}$ \\
\hline Havešová & $5,753 \mathrm{~m}$ & 16 & 50 & 50 \\
Kyjov & $3,051 \mathrm{~m}$ & 15 & 55 & 45
\end{tabular}

Canopy gaps in the upper canopy comprise approximately $15 \%$ of the forest area (Table 21), with extended gaps comprising $50 \%$ to $55 \%$. Only about half the forest area constituted closed stands. However the gap area decreases when gap closure is defined by trees with DBH $7 \mathrm{~cm}$ (Table 22), indicating that the percentage gap area is largely dependent on the defined threshold between gap and closed stand.

Tab 22. Percentage area of gaps with gap closure defined by trees with $7 \mathrm{~cm} \mathrm{DBH}$

\begin{tabular}{lcccc}
\hline & $\begin{array}{c}\text { total transect } \\
\text { length }\end{array}$ & $\begin{array}{c}\text { canopy } \\
\text { gaps [\%] }\end{array}$ & $\begin{array}{c}\text { extended } \\
\text { gaps [\%] }\end{array}$ & $\begin{array}{c}\text { closed } \\
\text { stand [\%] }\end{array}$ \\
\hline Havešová & $5,753 \mathrm{~m}$ & 7 & 21 & 79 \\
Kyjov & $3,051 \mathrm{~m}$ & 8 & 28 & 72
\end{tabular}




\subsubsection{Gap size distributions}

In Havešová and Kyjov Reserves 66 gaps, and 43 gaps were recorded along the transects respectively. This corresponded to 445 dead trees in Havešová, and 366 dead trees in Kyjov.

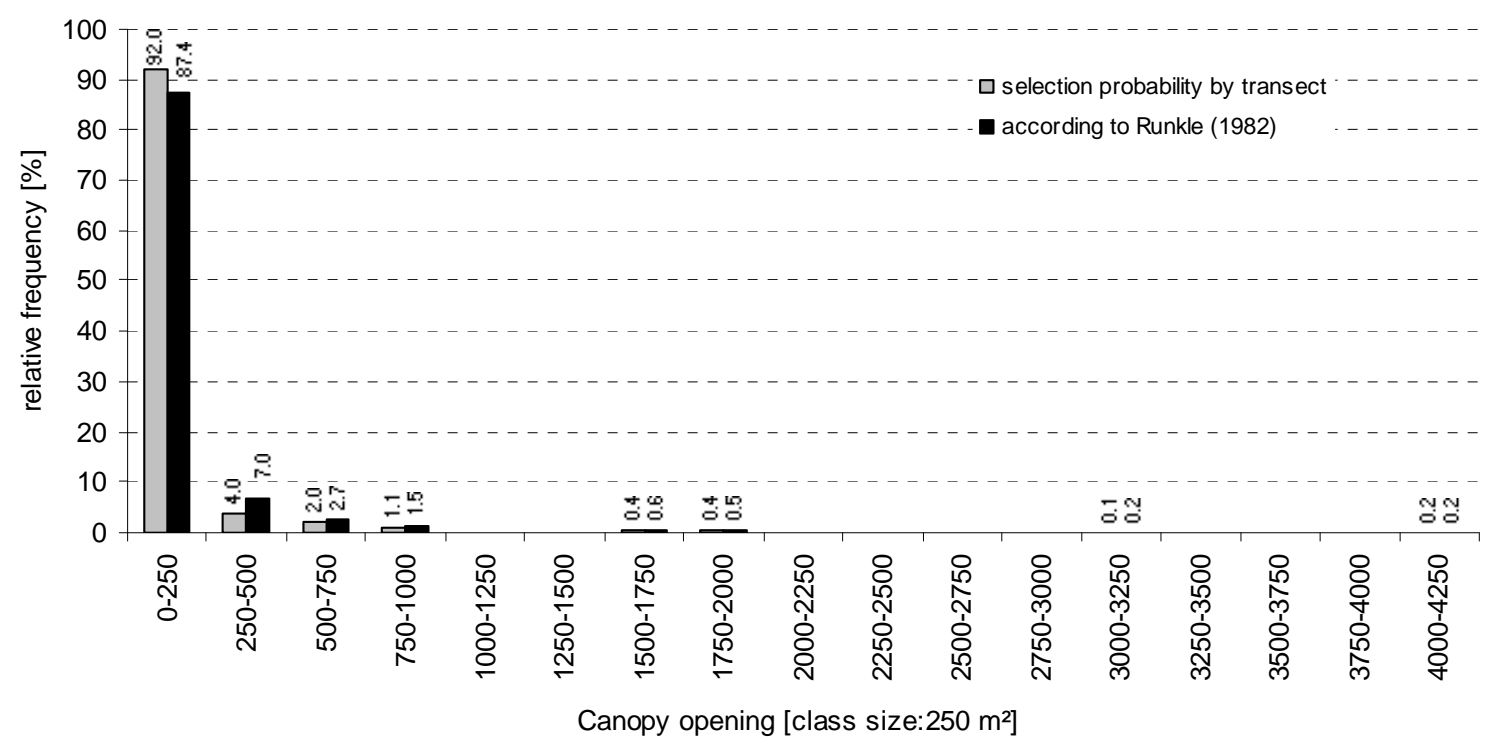

Fig. 18. Frequency of different sized canopy openings in Havešová, corrected as described in Chapter 4.4.3 and by Runkle (1982). A gap is defined as an opening in the canopy where regrowth is less than $2 / 3$ of the stand height.

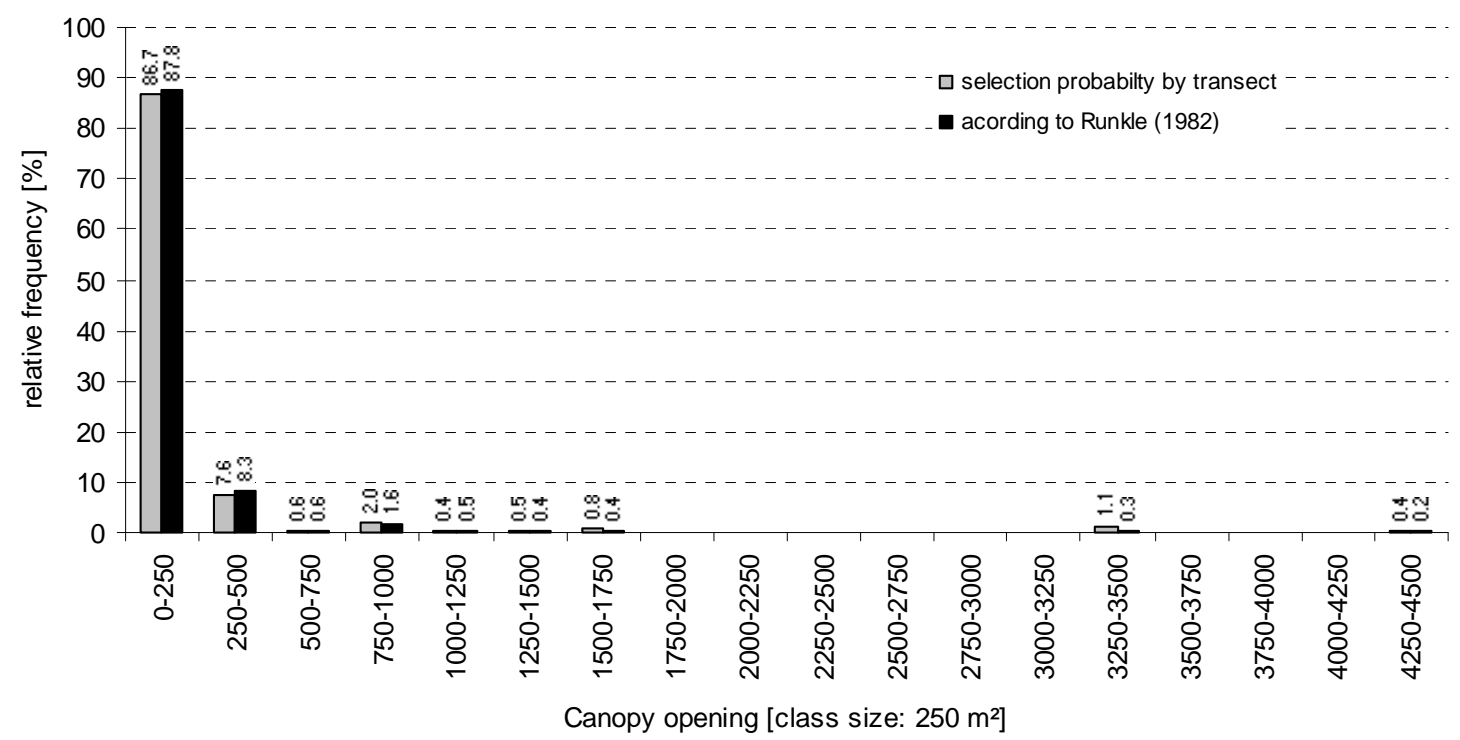

Fig. 19. Frequency of different sized canopy openings in Kyjov, corrected as described in Chapter 4.4.3 and by Runkle (1982). Gap defined as in Figure 18.

The two correction factors used in this study produced very similar results. More than $85 \%$ of the canopy gaps are smaller than $250 \mathrm{~m}^{2}$. Around $10 \%$ of gaps are openings between 250 and $1000 \mathrm{~m}^{2}$, and $1-3 \%$ of the gaps are larger than $1000 \mathrm{~m}^{2}$. If one divides the first gap class into 4 , about $75 \%$ of all gaps are smaller than $62.5 \mathrm{~m}^{2}$. The area of $7 \%$ of gaps ranges from $62.5-$ 
$125 \mathrm{~m}^{2} .4 \%$ of gaps have an area ranging from $125-187.5 \mathrm{~m}^{2}$, and $2 \%$ from $187.5-250 \mathrm{~m}^{2}$. The gap number decreases exponentially with increasing gap size.

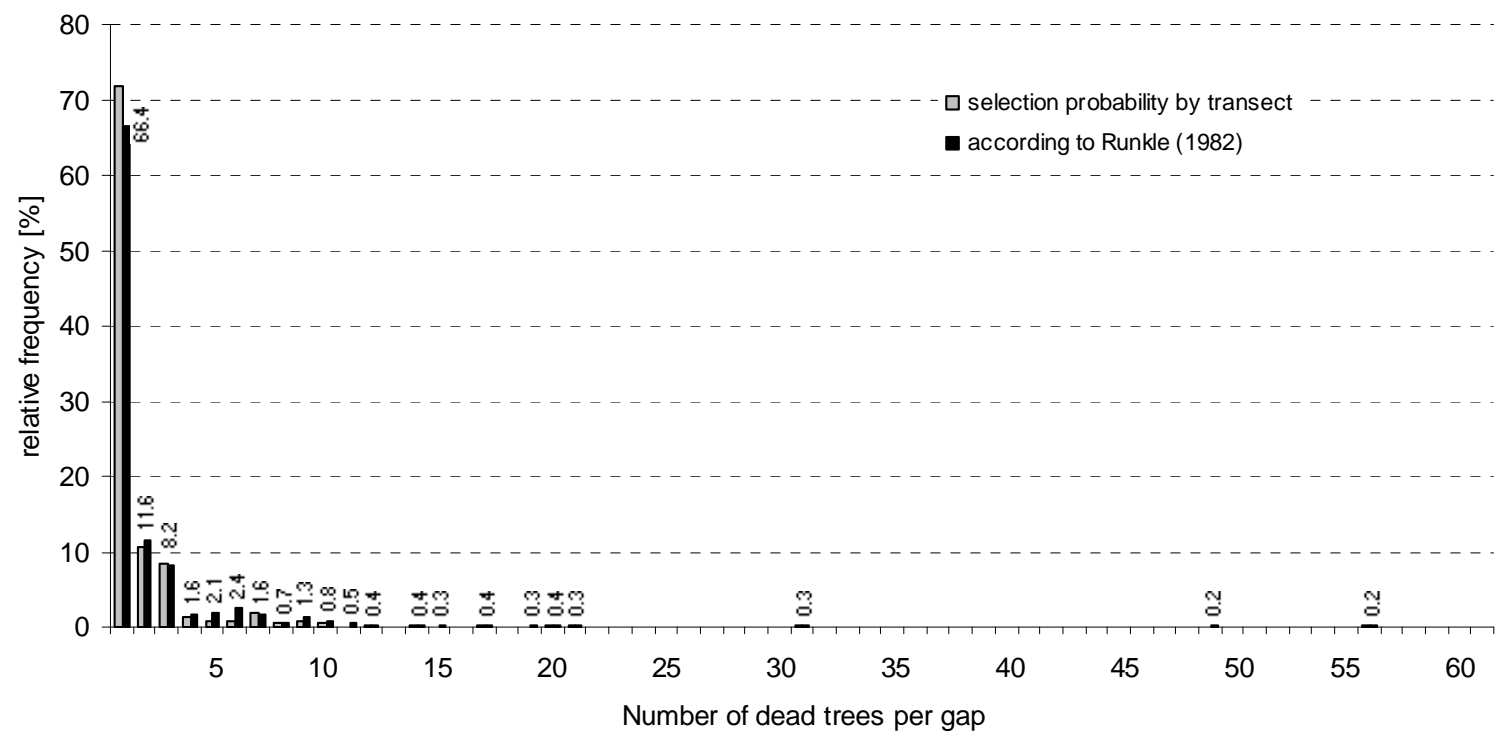

Fig. 20. Frequency of gaps in Havešová in relation to the number of dead trees per gap (Two correction methods are presented here and described in Chapter 4.4.3 and by Runkle, 1982; values according to Runkle are given.).

Two thirds of gaps are single-tree gaps and $20 \%$ are caused by the death of 2, 3 or 4 trees. As the gap creating trees were identified using woody debris analysis, a two-tree gap might be identified in which the first tree died 40 years ago and the second tree one year ago. About 3-5 $\%$ of gaps have more than 10 trees. Figure 20 and 21 depict clear exponential relationships between the number of dead trees per gap and relative frequency of gaps. More than 50 dead trees per canopy opening are very rare, but they do occur.

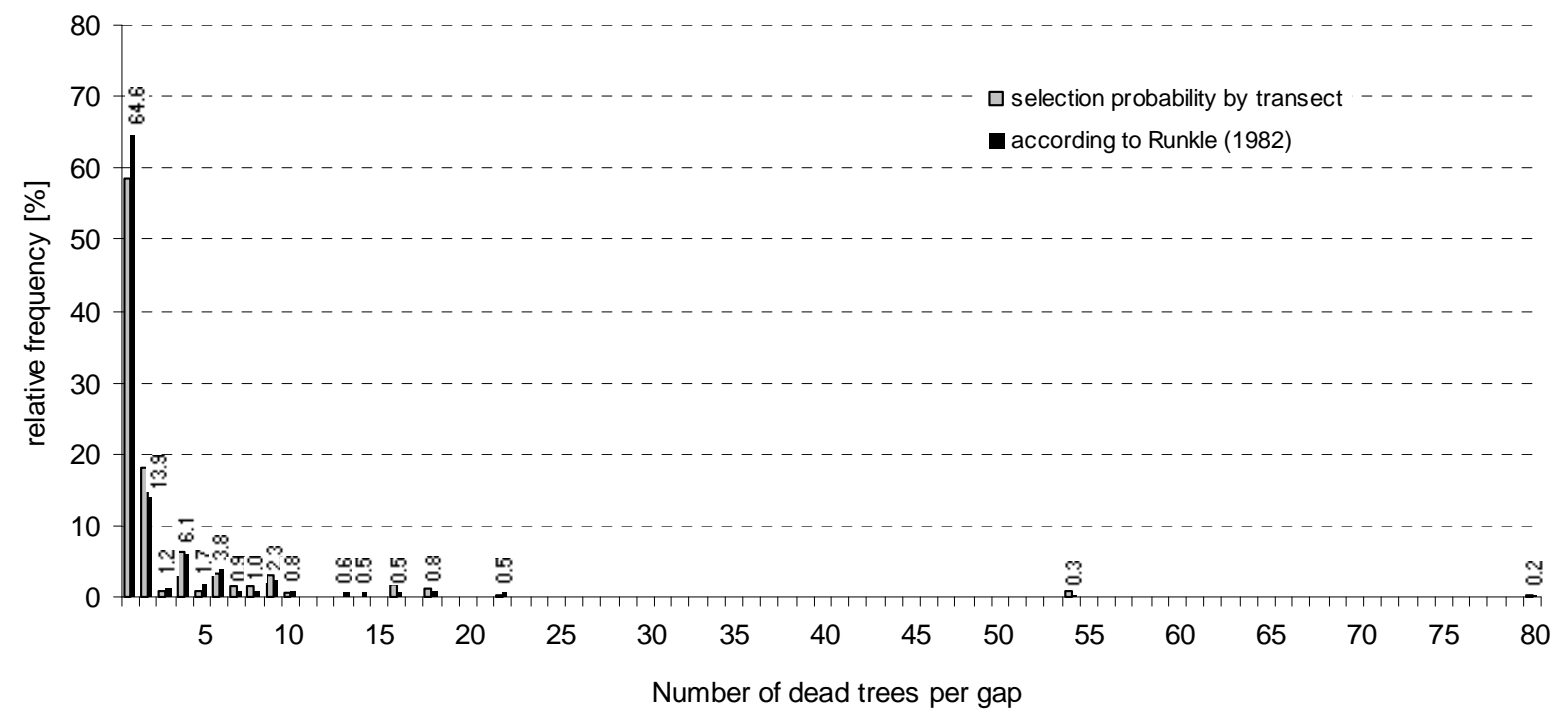

Fig. 21. Frequencies of gaps in Kyjov in relation to the number of dead trees per gap (Two correction methods were applied as in figure 20.). 
A reconstruction of the complete gap history is not possible. Former canopy trees that were completely decomposed were no longer visible and consequently neglected in the investigation. Time estimations of total decay of a canopy tree are presented in Chapters 4.5.5 and 4.6.3.

\subsubsection{Gap formation}

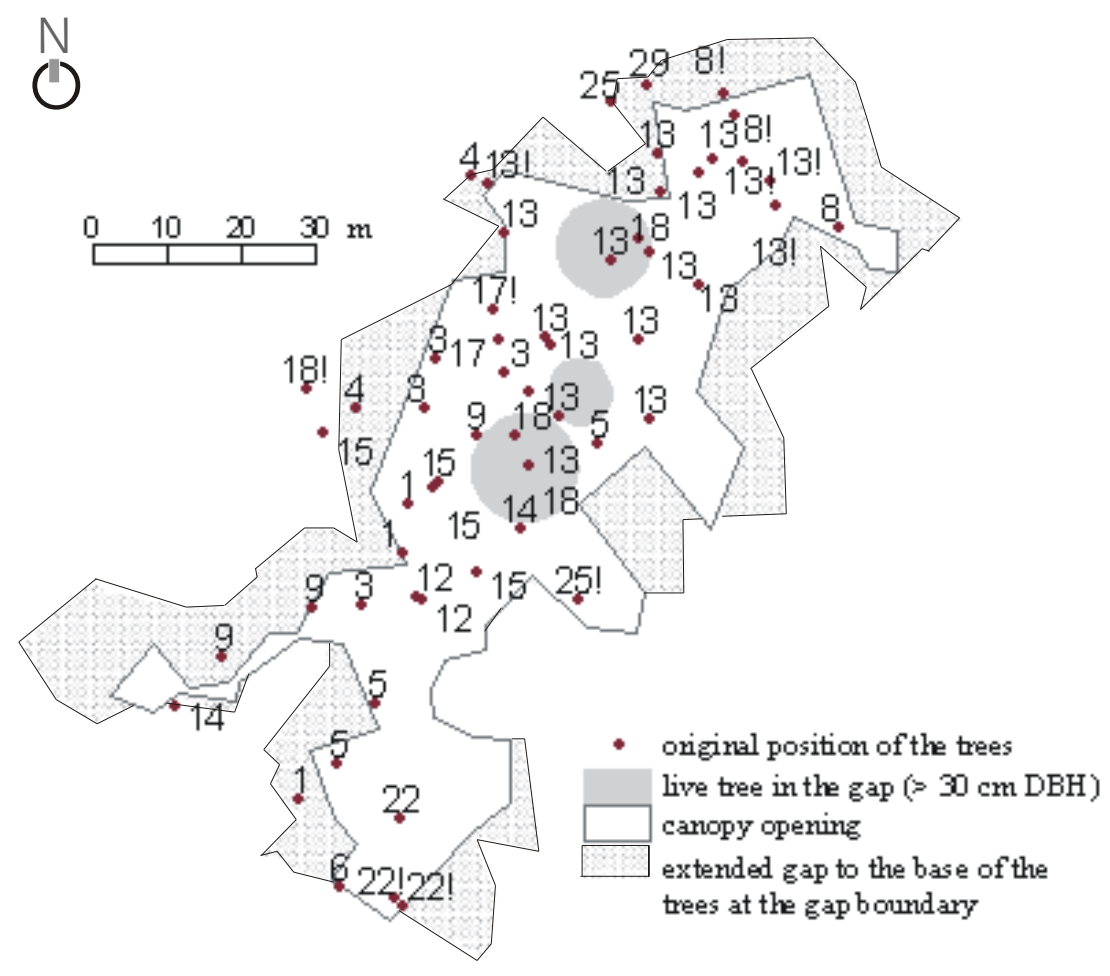

Fig. 22. Map of the largest gap along the transects in Havešová. The number adjacent to the original position of the trees is an estimation of the number of years the tree has been dead.

Cases where the timing of death is uncertain are identified with an exclamation mark.

An example of gap formation is provided in Figure 22, showing the history of the largest gap at Havešová. Formation patterns of large gaps can be interesting for silvicultural purposes. Eighteen years previously the gap consisted of small spot gaps. Around thirteen years ago most trees in the northeast section had been windthrown. In the southwest corner isolated small gaps joined more gradually.

Figure 23 shows the proportion of trees that have died in groups, or singly in all gaps at Havešová. More than half of the gap creating trees died singly. Groups with more than 12 trees occurred only once: 18 trees fell together in the largest gap, as seen in Figure 22.

Allowing for the uncertainties in dating, a gap was created suddenly (gap origin) by the death of $1 / 5$ of the trees and the gap was extended by $4 / 5$ of the trees dying. However, the mean age of single-tree gaps (22 years) and of the oldest gap creating trees in larger gaps (26 years) did 
not differ greatly, indicating a continuous extension of most small gaps. In large gaps with more than 20 gap creating trees the oldest gap creating trees were older than 20 years (see Figure 24).

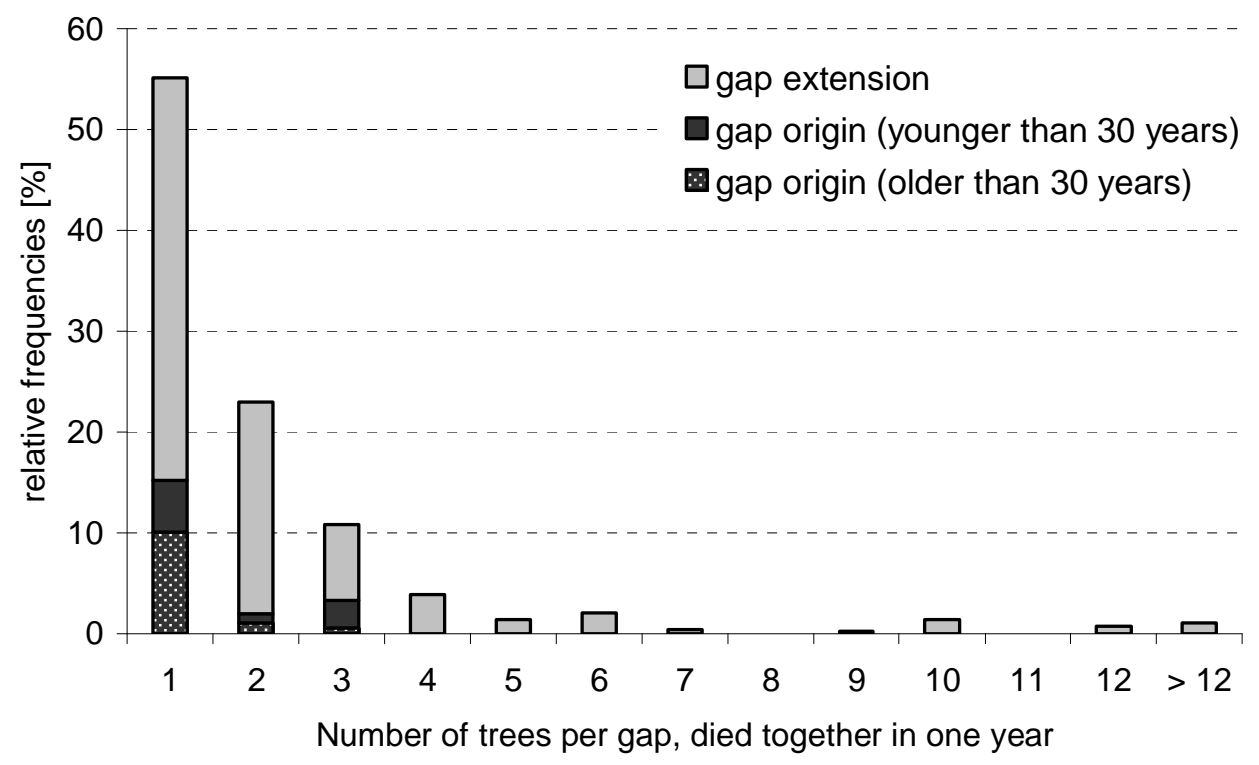

Fig. 23. Frequency of single or group of dead trees in each gap. (Dating the origin of gaps more than 30 years old is fairly unreliable due to rising probability of completely decomposed trees; correction for representativeness of trees in different sized gaps is described in 4.4.3.)

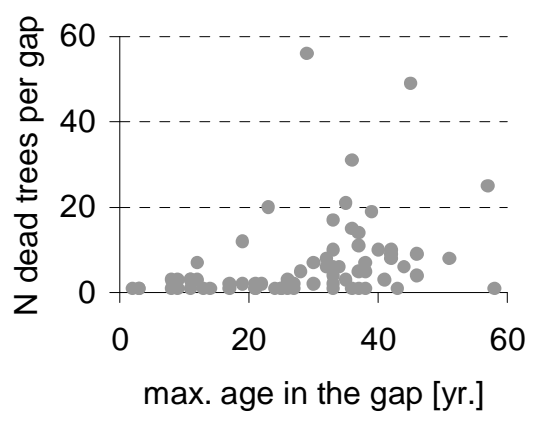

Fig. 24. Number of dead trees per gap in relation to the age of the oldest dead tree in the gap (without correction for the under-represented smaller, and over-represented larger gaps). 


\subsubsection{Causes of tree mortality}

In Havešová half the trees found in gaps died by uprooting. Often the trees had snapped off.

In Kyjov Reserve most trees died after breakage and 1/5 by uprooting. Standing dead trees were observed once in Havešová and five times in Kyjov.

Tab. 23. Percentages of mortality causes of canopy trees. (Values calculated representatively as described in 3.3.)

\begin{tabular}{lcccccc}
\hline & uprooting & $\begin{array}{c}\text { partial } \\
\text { uprooting }\end{array}$ & breakage & $\begin{array}{c}\text { standing } \\
\text { dead }\end{array}$ & $\begin{array}{c}\text { partial } \\
\text { dead }\end{array}$ & $\begin{array}{c}\text { No. of } \\
\text { dead trees }\end{array}$ \\
\hline Havešová & 49.1 & 3.9 & 44.9 & 0.1 & 2.0 & 445 \\
Kyjov & 20.8 & 1.6 & 72.9 & 1.5 & 3.2 & 366
\end{tabular}

The maximum DBH for dead trees was 140 cm in Havešová and $116 \mathrm{~cm}$ in Kyjov.

\subsubsection{Turnover time}

The time of death of trees in a gap varied, sometimes considerably. Thus the calculation of turnover time is based on estimated ages of dead trees as described in 4.4.5.

Tab. 24. Gap formation rate and turnover, calculated by gap percentages and proportion of trees dying less than 10 , and 20 years ago (* example of calculation in 3.5.; ** assumed monitoring period).

\begin{tabular}{|c|c|c|c|c|c|}
\hline \multirow[b]{2}{*}{ gap percentages (GP) } & \multicolumn{3}{|c|}{ Havešová } & \multicolumn{2}{|c|}{ Kyjov } \\
\hline & 16.02 & 16.02 & 16.02 & 14.63 & 14.63 \\
\hline $\begin{array}{l}\text { time period [years], } \\
\text { counted back }\end{array}$ & 10 & 20 & $35^{\star *}$ & 10 & $35^{* *}$ \\
\hline $\begin{array}{l}\text { percentage of } \\
\text { trees died in } \\
\text { this period (TP) }\end{array}$ & 24.18 & 66.04 & 100 & 31.21 & 100 \\
\hline GP $\times$ TP $/ 100^{*}$ & 3.87 & 10.58 & 16.02 & 4.56 & 14.63 \\
\hline $\begin{array}{l}\text { gap formation } \\
\text { rate [\% per year] }\end{array}$ & 0.387 & 0.529 & 0.458 & 0.456 & 0.418 \\
\hline turnover time [years] & 258 & 189 & 218 & 219 & 239 \\
\hline
\end{tabular}

The turnover time varies in relation to the chosen time period (Table 24). In our calculations we have assumed a monitoring period of 35 years, based on the estimated average period of decomposition of the trees. The method used to derive this estimate is explained in detail 
below. The turnover time in Havešová and in Kyjov was 218 years, and 239 years respectively. One might also assume a period of 30 (or 40) years on average before trees decompose completely, resulting in a turnover time of 187 (250) years at Havešová and 205 (273) years at Kyjov. Differences of 60-70 years exist because the average time of decomposition is not known exactly. Furthermore the turnover time was calculated for the last 10 years because dating the death of trees is most reliable in this period. In Havešová the turnover time in the last 10 years differs considerably from the turnover time in the last 20 years, because fewer trees died during last decade than in the previous decade (Figure 25).

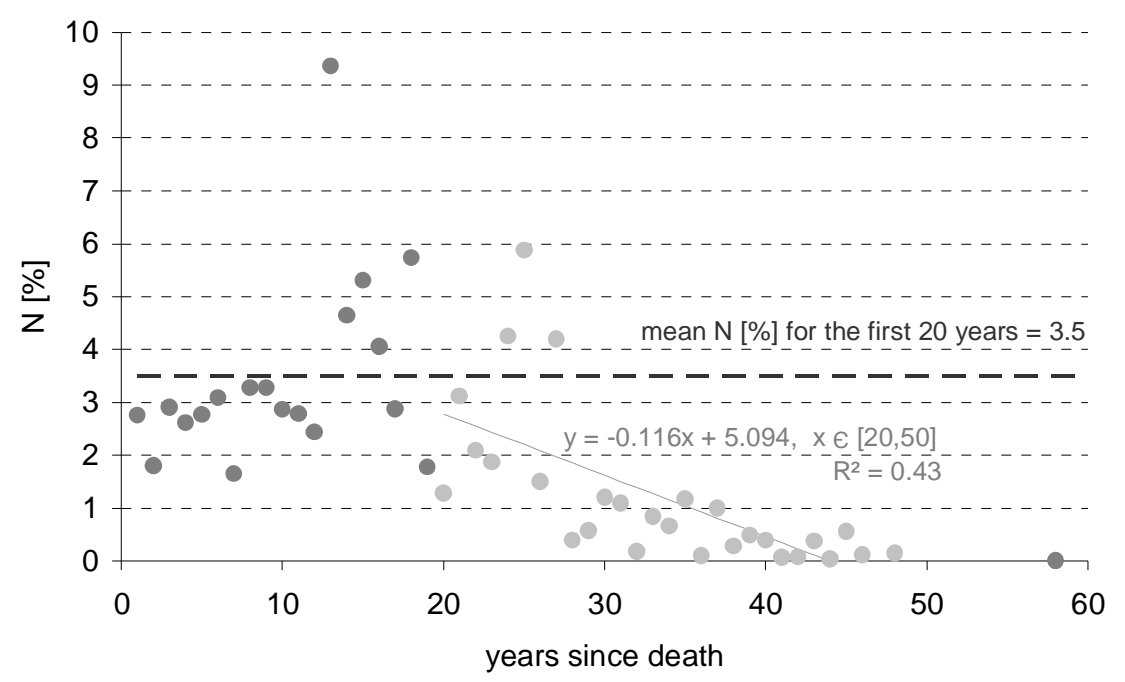

Fig. 25. Percentages of dead trees in relation to estimated time each tree has been dead. Values up to 20 years are most reliable (see 4.4.4.). Note that regression was performed for 20-50 years only.

A linear regression of trees dead for more than 20 years shows that a decreasing number of trees died between 20 and 50 years ago. It should be noted that the time required for complete decomposition of a tree, or the course of decomposition is unavailable. 20 years was adopted as the beginning of the regression since the complete decomposition of a canopy trees after 20 years is not possible. That may also be the case in later years. Here a linear decomposition is assumed to determine the average duration of decomposition of trees. For Havešová we estimate a mean period of 35 years for total decay of canopy trees, ranging from 23 to 58 years. The maximum duration of decomposition was estimated for a large tree, probably stored dry in $1.5 \mathrm{~m}$ height, although back-dating has an associated error of +/- 10 years. 


\subsection{Discussion}

Peters (1997) indicates that, in relation to growth rate increases of the stem diameter, greater dynamic changes occur in the canopy of beech forests in North America than in Europe. He maintains the high wind speed in southeastern USA and eastern Asia, in addition to the very high frequency of tornadoes in the USA are the cause. The disturbance rate in North American forests, comprising mixed forests with American beech, amounts to 0.5-2.0\% per year (Runkle 1982).

Turkish and Asian forests appear to be less dynamic (Peters, 1997). Yet even here SaghebTalebi and Delfan Abazari (2003) found a 1,680 $\mathrm{m}^{2}$ canopy opening. They stress the similarities between beech forests in the Orient and eastern Europe. In Japanese beech forests gap percentages range from 6.2 to $37.5 \%$ (Nakashizuka, 1988; Yamamoto and Nishimura, 1999). This may be due to the small study areas (1-6 ha) or it may support a distinction between species.

Although Runkle (1985) assumes that different mixed broadleaved forests probably do not exhibit large differences in their average rates of disturbance, here we restrict the discussion to Fagus sylvatica.

\subsubsection{Methodological aspects}

The spatial and temporal limitations in this investigation meant that large-scale disturbances in the landscape could not be detected. Thus conclusions about the naturalness of the silvicultural systems in this study, such as Seymour et al. (2002) propose, are strictly limited to disturbances causing the removal of single trees or groups of trees. Shelterwood harvesting methods were not adequately considered where information about gap density was unavailable. Large beech forests that have not been managed for timber extraction in the past still exist in the Ukraine and Romania. These forests, covering an area of about 10,000 ha, provide an opportunity to investigate large disturbances. In such investigations aerial surveys and digital surface models of the vegetation layer are useful for computing gap percentage, size distribution and gap density (Tanaka and Nakashizuka, 1997; Nuske and Nieschulze, 2004).

In this terrestrial survey we have opened a "time window" of 30-40 years, covering the period from death of trees to gap closure or the almost complete decomposition of trees. Although uneven-aged virgin forests should facilitate studies that are largely time independent, storm incidents are discrete and randomly distributed events in time (Bielec-Bakowska, 2003; Dobrovolny and Brazdil, 2003). A theoretical model of the dynamics of spatial structures in 
European beech forests by Neuert (1999) illustrates that upper canopy cover depends mainly on heavy storm events. However ice-damage may also affect the number of large gaps considerably (Standovar and Aszalos, 2001).

\subsubsection{Gap definition, percentages and sizes}

As young trees with different dimensions may occur in gaps an arbitrary boundary must be drawn between regeneration in the gap and the closed stand. The definition of a gap as an opening in the canopy in the upper height stratum is justified by the traditional division of a stand into 3 height strata (Leibundgut, 1956) and our interest in the recruitment of the overstorey. The definition of gaps as $>1 / 2$ stand height is more appropriate for comparisons with aerial surveys. While the distinction between regeneration in the gap and closed stand using DBH instead of tree height was adequate in Havešová, in Kyjov more tree crowns were damaged making the distinction by height measurements necessary.

The distinction between gap and non-gap represents a simplification of forest structure (Lieberman et al., 1989). However it was adhered to in this study for practical reasons. If a single branch touched a neighbouring tree the gap boundary was ruled out at this point. The percentage of gaps defined by $7 \mathrm{~cm} \mathrm{DBH}$ (Table 22) is provided for comparisons with Tabaku and Meyer (1999) and studies in even-aged forests (e.g. Schmidt, 1996). Zeibig et al. (2005) defined gaps by trees that had not reached half the stand height. The greater the DBH/height adopted to define threshold, the larger the gap becomes. Further research into percentage representation of development stages should involve the investigation of gaps defined by $10,20,30, \ldots \mathrm{cm}$ DBH along the same transect.

The percentage of gaps found by Tabaku and Meyer (1999) in three stands, and by Zeibig et al. (2005) was 3.3-6.6\%, and 5.6\% respectively. The values in this study exceed the range reported by Tabaku and Meyer slightly. When applying the gap definition above the gap percentage in the Slovenian forest (Zeibig et al., 2005) was found to be slightly lower than in the Albanian or Slovakian forests. In this study, by determining the differences between gap percentages using the different definitions (Table 21 and 22), half the gap area defined by $2 / 3$ stand height was covered by trees with a diameter of at least $7 \mathrm{~cm}$.

In addition to assessing the percentage area of gaps line transect sampling also allows the derivation of a representative frequency distribution for different sized gaps (Runkle, 1992). Despite the non-representative collection of data along the transects, a representative distribution can be obtained. In this study the two independent correction methods were found to be consistent (Figure 16-19). 
Tabaku and Meyer (1999) identified a mean gap size of 61-74 $\mathrm{m}^{2}$ with a range in gap size from 20 to $270 \mathrm{~m}^{2}$, based on 3.6, 5 and 6 ha plots. The average was strongly influenced by a few large gaps. Zeibig et al. (2005) created 3/4 gaps less than $200 \mathrm{~m}^{2}$ and $9 \%$ gaps between 200-600 $\mathrm{m}^{2}$. This size distribution (with $6 \mathrm{~m}^{2}$ minimum gap area), comprising 49 gaps on a 12 ha study plot, is very similar to Figure 16 and 17. A more detailed comparison of the three studies is provided in Drößler and Lüpke (2005b).

Our sample included all gaps along the transects. Consequently the recording limit could be defined after sampling. Gaps smaller than $1 \mathrm{~m}^{2}$ were not found, which may be due to the method used.

It is interesting to note that Zeibig et al. (2005) indicated that gaps between 200-600 $\mathrm{m}^{2}$ take up more land area than gaps $<200 \mathrm{~m}^{2}$. In Havešová gaps greater than $535 \mathrm{~m}^{2}$ comprise half the total gap area. In Kyjov gaps greater than $875 \mathrm{~m}^{2}$ comprise half the total gap area. In this study there was a greater proportion of large gaps in the total gap area but a greater number of smaller gaps. Thus in Havešová $80 \%$ of all gaps are smaller than $130 \mathrm{~m}^{2}$, corresponding to the mean crown cover of a canopy tree with $60 \mathrm{~cm} \mathrm{DBH}$. A tree with $80 \mathrm{~cm}$ diameter has a projected crown cover of about $200 \mathrm{~m}^{2}$.

The forest reserve "Heilige Hallen" in Germany has been unmanaged for 150 years and comprises 250 years old beech trees. In this old even-aged stand the scale of decay processes in the canopy openings appear to be similar to those in natural forests: $13.3 \%$ gaps (boundary between closed stand and gap defined by trees with $35 \mathrm{~cm} \mathrm{DBH}$; Tabaku and Meyer, 1999). Gap size distributions in 120-160 years old beech stands that have not been managed for 1118 years are similar to that in Figure 3 and 4 (Richter, 1990). Richter assumed a gap creation rate of about $0.2 \%$ per year in these natural forest reserves. Manning and Smaltschinski (2001) calculated a gap percentage of only $1.9 \%$ in an un-evenaged beech stand that was unmanaged for 50 years. The fewer disturbance events may be explained by the younger tree age compared to the oldest trees in virgin forests.

\subsubsection{Age estimation, gap formation and turnover time}

The age of dead trees in this study has been estimated rather than accurately measured. Yet this estimate is more useful for silvicultural purposes than assessing decay stages, described by qualitative features only. In view of Figure 23, 24 and 25 estimations over 30 years old should be interpreted carefully. 
In Figure 25 we can estimate the mean decomposition time of dead trees. If one assumes a constant number of dead trees in virgin forests over time and a more or less constant removal of these trees through decomposition, then the duration of this time period is estimated to be 35 years on average. Saniga and Schütz (2002) report a decomposition period of 30-35 years in virgin beech forests.

In extreme cases gaps that were created up to 58 years ago were found. However most of those gaps are now assumed closed. Scientists have estimated the average gap turnover time, basing determinations on the average time required for gap closure, to be (30-) 35 (-40) years. The turnover time calculated corresponds to the maximum life expectancy of 220 years (sometimes 250 years) of beech trees in Kyjov Reserve (Korpel, 1967). Upon consideration of the different turnover times obtained for the monitoring periods of 10 or 20 years in this study, the turnover time for Havešová and Kyjov was estimated to be $220+/$ - 30 years (Table 4). In Denmark Emborg et al. (2000) found that the complete forest cycle of a near-natural beech stand was a few decades longer, with a turnover time of 284 years. This may be due to the stand history. Individual trees released in managed stands may also exceed 300 years. In addition Korpel (1967) identifies mean ages of trees in closed stands of 39, and 95 years in the lower and middle height stratum respectively. Although trees grow faster in gaps it is unrealistic to expect a $30 \mathrm{~cm}$ DBH after 35 years. In almost every gap at least one tree in the middle height stratum survived the canopy tree fall, explaining accelerated gap closure. Adjacent canopy trees also contribute to gap closure according to Schmidt (1996) and Pedersen and Howard (2004).

Upon further consideration of the development stages in forests the presence of a large number of dead trees, which have expanded gaps further, supports the theory of shifting mosaics (Watt, 1947) (Figure 23). Figure 24 also shows, however, that a large portion of the single-tree gaps did not increase in size.

Gaps can also been regarded as initial stages of development. Korpel' (1967) identified isolated stages in Havešová und Kyjov in areas 0.5 ha in size. Differences certainly occur in the size distribution between gaps and later development stages although an exponential distribution is also probable. Isolated patches with an area of 0.5 ha are probably one of the less frequently occurring stages. If one adopts the theory of shifting mosaics then the probability that these patches meet the more frequent smaller patches increases over time. For this reason we regard the documentation of the tree coordinates to be a useful addition to the 
valuable long-term studies in Slovakia, to assist the implementation of further research on development stages in the future.

\subsection{References}

Bielec-Bakowska, Z. (2003): Long-term variability of thunderstorm occurrence in Poland in the 20th century. Atmospheric research 67-68: 35-52.

Dobrovolny, P. and Brazdil, R. (2003): Documentary evidence on strong winds related to convective storms in the Czech Republic since AD 1500. Atmospheric research 67-68: 95-116.

Drößler, L. and Lüpke, B.v. (2005b): Forest gaps in virgin beech forest in Havešová Reserve. In: Hamor, F.D. and Commarmot, B. (eds): Natural forests in the temperate zone of Europe - Values and utilisation. International conference in Mukachevo, Ukraine. October 13-17, 2003. Rakhiv, Carpathian biosphere Reserve; Birmensdorf, Swiss Federal Research Institute WSL.

Emborg, J., Christensen, M. and Heilmann-Clausen, J. (2000): The structural dynamics of Suserov Skov, a nearnatural temperate deciduous forest in Denmark. For. Ecol. Manage. 126: 173-189.

Standovar, T. and Kenderes, K. (2003): A review on natural stand dynamics in beechwoods of East Central Europe. Applied ecology and environmental research 1(1-2): 19-46.

Korpel', Š . (1967): Development and age structure of the virgin beech forest in Vyhorlat-mountains (Kyjov). Biologia 22(4): 285-303. Slovak.

Korpel', Š. (1995): Die Urwälder der Westkarpaten. Stuttgart, Jena, New York: Gustav Fischer Verlag. 310 p. German.

Leibundgut, H. (1956): Empfehlungen für die Baumklassenbildung und Methodik bei Versuchen über die Wirkung von Waldpflegemaßnahmen. IUFRO Sekt. 23. 10. report. German (French summary).

Lieberman, M., Lieberman, D., and Peralta, R. (1989): Forests are not just Swiss cheese: Canopy

Stereogeometry of non-gaps in tropical forests. Ecology, 70(3): 550-552.

Manning, D.B. and Smaltschinski, T. (2001): Natural gaps in a beech forest in Central Germany. In: Mountford, E.P. (eds.): Natural canopy gap characteristics in European beech forests. NatMan project WP2. Working Report 6. Deliverable 20.

Nakashizuka, T. (1988): Gap formation and species diversity of beech forests in Japan. Proceedings of 3th IUFRO beech symposium in Zvolen. pp. 169-181.

Neuert, C. (1999): Die Dynamik räumlicher Strukturen in naturnahen Buchenwäldern Mitteleuropas. UFZBericht No. 20/1999. 186 p. German.

Nuske, R. and Nieschulze, J. (2004): Die Vegetationshöhe als Werkzeug zur Ermittlung von Bestandeshöhen. Eine Anwendung automatisierter digitaler Photogrammetrie in der Forstwissenschaft. Allg. Forst- u. J.-Ztg. 175(1/2): 13-21. German.

Pedersen, B.S. and Howard, J.L. (2004): The influence of canopy gaps on overstory tree and foresth growth rates in a mature mixed-age, mixed-species forest. For. Ecol. Manage. 196: 351-366.

Peters, R. (1997): Beech forests. Geobotany 24. Kluwer academic publishers.Dordrecht. Boston. London.

Richter, J. (1990): Stammbruch, Windwurf und Naturverjüngung in Buchen-Naturwaldzellen. Schriftenreihe der LÖLF 12: 86-96. German.

Runkle, J.R. (1982): Patterns of disturbance in some old-growth mesic forests of eastern North America. Ecology, 63(5): 1533-1546.

Runkle, J.R. (1982): Patterns of disturbance in some old-growth mesic forests of eastern North America. Ecology 63: 1533-1546.

Runkle, J.R. (1985): Disturbance regimes in temperate forests. In: Pickett, S.T.A. and White, P.S. (eds.): The ecology of natural disturbance and patch dynamics, p. 17-33. Academic press, Orlando.

Runkle, J.R. (1992): Guidelines and Sample Protocol for Sampling Forest Gaps. Forest Service, General Technical Report PNW-GTR-283.

Sagheb-Talebi, Kh. and Delfan Abazari, B. (2003): Regeneration Process in Natural Uneven-aged Caspian Beech Forests of Iran. IUFRO International Interdisciplinary Conference and Field Tour in Finland and Sweden, June 8-17, 2003. Uneven-aged Forest Management: Alternative Forms, Practices and Constraints. Presented by Metla (Finnish Forest Research Institute), Collection of Abstracts (presented on June 10, 2003), 5 p.

Schmidt, W. (1996): Zur Entwicklung der Verjüngung in zwei Femelücken in einem Kalkbuchenwald. Forst u. Holz 51: 201-205. German.

Saniga, M. and Schütz, J.P. (2002): Relation of dead wood course within the development cycle of selected virgin forests in Slovakia. J. For. Sci., 48(12): 513-528.

Seymour, R.S., White, A.S. and deMaynadier, P.G. (2002): Natural disturbance regimes in northeastern North America - evaluating silvicultural systems using natural scales and frequencies. For. Ecol. Manage. 155: 357367. 
Standovar, T. and Aszalos, R. (2001): Natural gaps in beech forests in Hungary. In: Mountford, E.P. (eds.): Natural canopy gap characteristics in European beech forests. NatMan project WP2. Working Report 6. Deliverable 20.

Tabaku, V. and Meyer, P. (1999): Lückenmuster albanischer und mitteleuropäischer Buchenwälder unterschiedlicher Nutzungsintensität. Forstarchiv, 70: 87-97. German.

Tanaka, H. And Nakashizuka, T. (1997): Fifteen years of canopy dynamics analyzed by aerial photographs in a temperate deciduous forest, Japan. Ecology 78(2): 612-620.

Watt, A.S. (1947): Pattern and process in the plant community. J. Ecol.35: 1-22.

Yamamoto, S. and Nishimura, N. (1999): Canopy gap formation and replacement pattern of major tree species among developmental stages of beech (Fagus crenata) stands, Japan. Plant ecology 140: 167-176.

Zeibig, A.; Diaci, J.; Wagner, S., (2005): Gap disturbance patterns of a beech virgin forest remnant in the mountain vegetation belt of Slovenia. In: Hamor, F.D. and Commarmot, B. (eds): Natural forests in the temperate zone of Europe - Values and utilisation. International conference in Mukachevo, Ukraine. October 1317, 2003. Rakhiv, Carpathian biosphere Reserve; Birmensdorf, Swiss Federal Research Institute WSL. 


\section{Waldentwicklungsphasen}

Dieser Teil entspricht einer Publikation von L. Drößler und P. Meyer im Jahr 2006:

Waldentwicklungsphasen in zwei Buchen-Urwaldreservaten in der Slowakei. Forstarchiv 77: $155-161$.

\subsection{Kurzfassung}

Die Anteile unterschiedlicher Waldentwicklungsphasen in zwei Buchen-Urwaldreservaten wurden bestimmt. Mehr als 70 Prozent der Waldfläche wurden von der Plenter-, Terminalund Zerfallsphase eingenommen. Die Optimalphase nahm ungefähr 15 Prozent ein. Diese Verteilung wurde bereits in albanischen Buchenurwäldern beobachtet und unterscheidet sich deutlich vom Wirtschaftswald.

In den einzelnen Waldentwicklungsphasen wurden das Holzvolumen und die Stammzahl ermittelt und untereinander verglichen. Das durchschnittliche Holzvolumen betrug in Havešová 720 m³/ha. Es schwankte je nach Phase zwischen $100 \mathrm{~m}^{3} / \mathrm{ha}$ und $1000 \mathrm{~m}^{3} / \mathrm{ha}$. In Kyjov betrug das durchschnittliche Holzvolumen $450 \mathrm{~m}^{3} / \mathrm{ha}$ und erreichte in der Plenterphase 670 m³/ha. Das Totholzvolumen schwankte in Havešová zwischen 14 und 490 m³a und in Kyjov zwischen 7 und 320 m³ha. Die Dichte der Bäume betrug je nach Phase 30 bis 400 Stück/ha. Die jüngste Entwicklungsphase (Lücke) trat aber nur sehr kleinflächig und vereinzelt auf.

Einen weiteren Schwerpunkt der Untersuchung bilden methodische Überlegungen zur minimalen Flächengröße der einzelnen Phasen. Dabei wird ein Ansatz vorgestellt, der den einzelnen Baum und seine Lage als Grundeinheit für Analysen des Waldaufbaus stärker berücksichtigt.

Schlagwörter: Walddynamik, Entwicklungsphasen, Urwald, Störungen, Fagus sylvatica

\subsection{Abstract}

Title: Forest development stages in two virgin beech reserves in Slovakia. The area proportion of different forest development stages in two virgin beech forests was determined. Almost the half of forest area comprised the terminal- and decay stage. The optimum stage covered about $15 \%$. This distribution was also observed in virgin beech forests in Albania and differs clearly from managed stands.

During single stages the timber volume and tree number was determined and compared with each other. The mean timber volume in Havešová was 720 m³/ha. It amounted during the 
stages from $100 \mathrm{~m}^{3} / \mathrm{ha}$ to $1000 \mathrm{~m}^{3} / \mathrm{ha}$. In Kyjov the mean volume amounted to $450 \mathrm{~m}^{3} / \mathrm{ha}$ and increased to $670 \mathrm{~m}^{3} / \mathrm{ha}$ during the plenter stage. Deadwood volume amounted from 14 to 490 $\mathrm{m}^{3} / \mathrm{ha}$ in Havešová and in Kyjov from 7 to $320 \mathrm{~m}^{3} / \mathrm{ha}$. Tree density varied from 30 to 400 trees per ha among the stages. But the youngest stage occured sparsely on small areas.

A further focus of this work was a methodological aspect to determine the minimum size area of single stages. With it an attempt is presented, that considers stronger the single tree and its location as basal unit to analyse stand structure.

Key words: forest dynamic, development stages, virgin forest, disturbances, Fagus sylvatica

\subsection{Einleitung}

Waldentwicklungsphasen sind ein wichtiges analytisches und didaktisches Instrument, um langfristige Veränderungen im Waldaufbau zu erkennen. Ausgehend von dem ökologischen Konzept der Sukzession bot sich für den Wald als Endstadium eine weitere Unterteilung dieses Stadiums in Phasen an (Leibundgut 1993). Die Kartierung der Phasen erfolgte in der Vergangenheit in vielen Fällen gutachterlich im Wald und war nicht in jedem Fall eindeutig (Neumann 1979, Knapp und Jeschke 1991, Leibundgut 1993). Die Reproduzierbarkeit der Ergebnisse war nicht gewährleistet. Aus diesem Grund entwickelte Meyer (1999) ein Verfahren zur quantitativen Bestimmung der Waldentwicklungsphasen, welches auf waldwachstumskundlichen Parametern eines Waldes basiert und diese kleinflächig analysiert. Dadurch konnten für Naturwaldreservate Waldentwicklungsphasen ausgeschieden werden, die miteinander vergleichbar sind und langfristige Beobachtungen erlauben (Meyer 1999). Ein ähnliches Verfahren entwickelte Tabaku (1999) für den Vergleich von Urwäldern mit Wirtschaftswäldern.

Ziel dieser Untersuchung ist es, dieses Verfahren auf zwei weitere Urwaldbestände anzuwenden, das methodische Konzept zu hinterfragen und einen anderen Ansatz zur Beschreibung der Waldtextur vorzustellen.

\subsection{Material und Methoden}

Die Untersuchung beschreibt zwei Buchenurwaldreservate im Osten der Slowakei. Das Reservat Havešová liegt in den Beskiden in einer Höhe von 500-650 m ü. NN. Es ist zum Süden exponiert und umfasst eine Fläche von 170 ha. Der jährliche Niederschlag schwankt zwischen 700-800 mm (450 mm in der Vegetationsperiode). Die mittlere Jahrestemperatur beträgt $7{ }^{\circ} \mathrm{C}$. Das Klima ist etwas kontinentaler geprägt als in Deutschland. Das geologische 
Ausgangssubstrat wird in Havešová von Flysch gebildet, über dem sich eine Braunerde entwickelt hat.

Das Reservat Kyjov liegt im Vyhorlat-Gebirge (700-820 m ü. NN). Es ist nach Norden geneigt und umfasst 53 ha. Der jährliche Niederschlag liegt bei 750-800 mm und die Jahresdurchschnittstemperatur beträgt $6{ }^{\circ} \mathrm{C}$. Das Ausgangsgestein ist Andesit, auf dem sich ebenfalls eine Braunerde befindet. Es handelt sich in beiden Fällen um reine Buchenwälder. Beschreibungen zum Waldaufbau geben Korpel' (1995) und Drößler und Lüpke (2005). Informationen zum Standort und zu den Wuchsverhältnissen geben Drößler und Lüpke (2006).

Die Aufnahme der Bäume ab 7 cm Brusthöhendurchmesser (BHD) erfolgte auf 22 bzw. 20 systematisch verteilten Stichprobenflächen. Der Mindestabstand zur Reservatsgrenze betrug 100 m. Die Stichprobenflächen waren Quadrate mit 62,5 m Kantenlänge. Auf dieser Fläche wurden für jeden Baum die Lagekoordinaten und der BHD bestimmt. Je Reservat wurden an 60 gleichmäßig über das Durchmesserspektrum verteilten Bäumen die Baumhöhe und acht Kronenradien gemessen. Die Radien wurden gemittelt. Auf Grund eines engen Zusammenhangs zwischen BHD und Kronenradius (Drößler und Lüpke 2006) konnte jedem Baum eine kreisförmige Krone zugeordnet werden, und eine Kronenkarte erstellt werden. Das Holzvolumen des lebenden Bestandes wurde mit Hilfe der Volumenfunktion von Petráš und Pajtík (1991) bestimmt. Totholzobjekte mit mehr als $20 \mathrm{~cm}$ BHD oder $20 \mathrm{~cm}$ Enddurchmesser sowie mehr als 2 m Länge wurden eingemessen und die Durchmesser aufgenommen. Stärker zersetzte Stämme wurden mit visuell geschätzten Volumenabschlägen versehen. Die einzelnen Stichprobenflächen wurden in 25 kleine Quadrate á 12,5 m x 12,5 m geteilt, auf denen der Deckungsgrad der Verjüngung geschätzt wurde.

Ein Verfahren für die Ausscheidung von Waldentwicklungsphasen in Naturwaldreservaten beschreibt Meyer (1999). Die Ausscheidung basiert auf der Veränderung des Derbholzvolumens eines Bestandes zwischen zwei unterschiedlichen Aufnahmezeitpunkten. Für Urwälder wurde von Tabaku (1999) ein Verfahren entwickelt, das sich an dem Verfahren von Meyer orientiert. Es basiert aber nur auf einer einmaligen Aufnahme. Dieses Verfahren wurde auch in der vorliegenden Untersuchung verwendet. Das Verfahren von Tabaku (1999) gewährleistet einen räumlichen Bezug der waldwachstumskundlichen Parameter. Bei der Auswertung bezieht man sich auf eine Fläche von 12,5 m x 12,5 m $\left(=156 \mathrm{~m}^{2}\right)$, die der Kronenschirmfläche einer Altbuche ungefähr entspricht. Als Entscheidungskriterien werden folgende Parameter herangezogen (siehe Abb. 1): Maximaler BHD $\left(\mathrm{d}_{0}\right)$ und Baumhöhe $\left(\mathrm{H}_{0}\right)$ 
auf einem Quadrat, Flächenanteil der Naturverjüngung auf einem Quadrat (NVF), Summe der Kronenschirmfläche der Bäume über $7 \mathrm{~cm}$ BHD auf einem Quadrat (KSF), Totholzanteil auf einem Quadrat (THA), Durchmesserspektrum aller Bäume auf einem Quadrat (normierter Quartilabstand) und die Entwicklungsstadien der Verjüngungsflächen (Dickung, Gertenholz, Stangenholz).

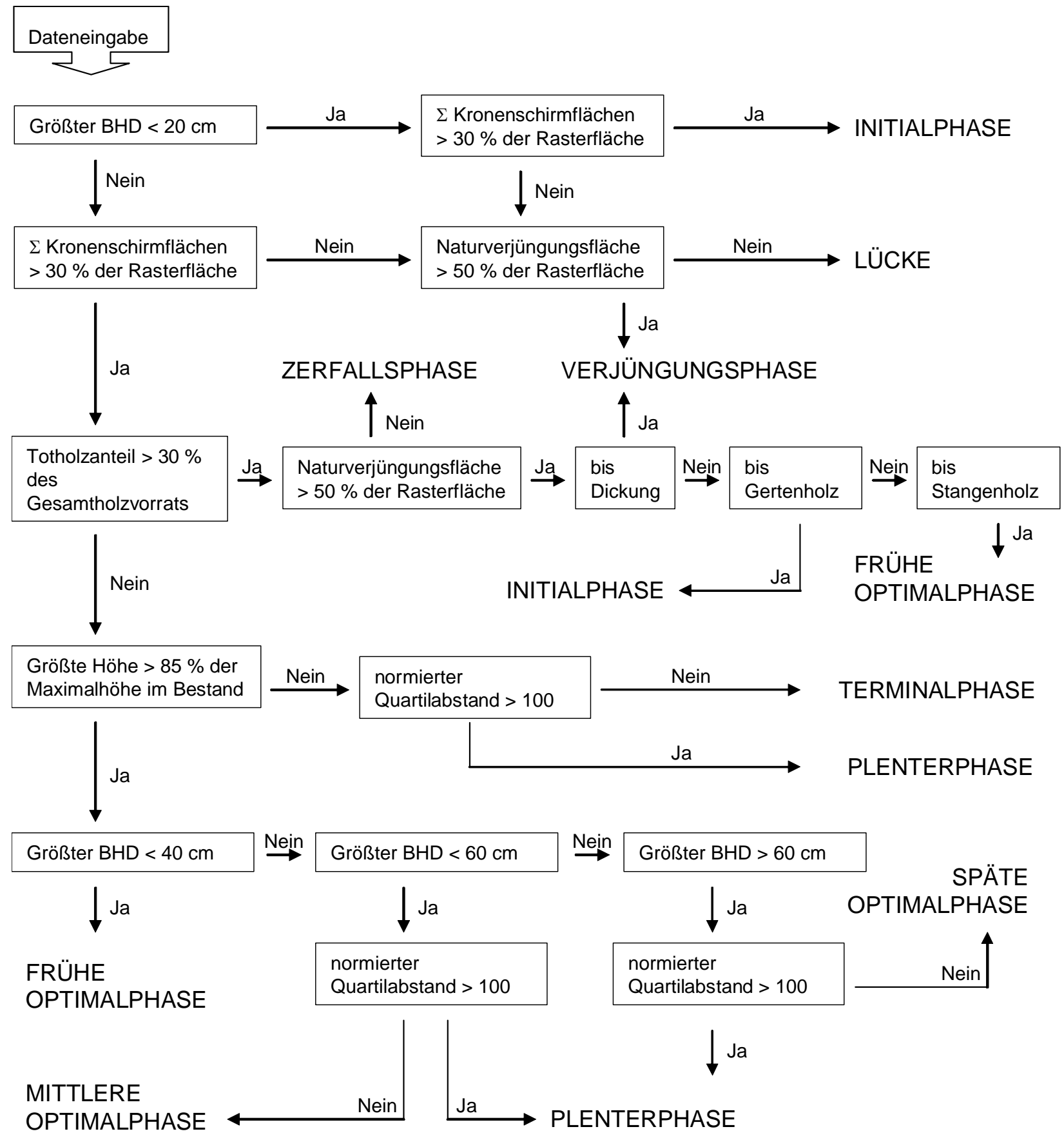

Abb.26 Flussdiagramm für die Ausscheidung der Waldentwicklungsphasen (Tabaku 1999) 
Im zweiten Teil der Arbeit wird ein Ansatz von Grassi (2003) weiterverfolgt, der die genaue Lage der einzelnen Bäume und ihre Nachbarschaft stärker berücksichtigt. Er basiert wie die vorigen Verfahren auf Stammverteilungsplänen der Bäume. Neben der Lage der Bäume finden aber nur BHD und Kronenradius Eingang in die Berechnung. In einem ersten Schritt werden die Bäume in BHD-Klassen eingeteilt, die ungefähr den Dimensionen bestimmter Phasen entsprechen. So lässt sich die frühe, mittlere und späte Optimalphase in Bäume von 21-40, 41-60 und 61-100 cm BHD einteilen. Die Initialphase umfasst Bäume von 7-20 cm BHD. Diese Definitionen sind gut mit den Definitionen von Meyer (1999) und Tabaku (1999) vergleichbar. Über den Kronenradius der Bäume kann entschieden werden, ob sich die Kronen der Bäume berühren. Ist das der Fall, werden die Bäume als Nachbarn betrachtet. Die Gruppen benachbarter Bäume einer Durchmesserklasse können dann ausgezählt werden. Die Anzahl der Bäume je Gruppe wird ebenfalls bestimmt. Das Verfahren wird von Drößler und Lüpke (2006b) beschrieben.

\subsection{Ergebnisse}

5.5.1 Waldentwicklungsphasen nach dem Verfahren von Tabaku (1999)

Tab. 25 Anteile [\%] der Waldentwicklungsphasen an der Gesamtfläche der Urwaldreservate, ausgeschieden nach dem Verfahren von Tabaku (1999)

\begin{tabular}{r|c|c} 
Waldentwicklungsphase & Havešová & Kyjov \\
\hline Lücke & 1,5 & 2,8 \\
Verjüngungsphase & 2,6 & 6,8 \\
Initialphase & 6,0 & 4,6 \\
Frühe Optimalphase & 8,9 & 8,2 \\
Mittlere Optimalphase & 4,7 & 4,0 \\
Späte Optimalphase & 3,8 & 0 \\
Plenterphase & 27,1 & 23,8 \\
Terminalphase & 29,6 & 36,6 \\
Zerfallsphase & 15,8 & 13,2
\end{tabular}

Die einzelnen Entwicklungsphasen besaßen in beiden Reservaten ähnliche Flächenanteile (Tabelle 25). Die Plenter- und Terminalphase nahmen zusammen etwa 60 \% der Fläche ein. Die Optimalphase nahm in Havešová 17,4 \% und in Kyjov 12,2 \% ein. Während sich die Anteile der frühen und mittleren Optimalphase ähnelten, wurde die späte Optimalphase in Kyjov gar nicht ausgewiesen. Der Anteil der beiden jüngsten Phasen war in Kyjov größer. Er betrug in Havešová $4 \%$ und in Kyjov knapp $10 \%$. 


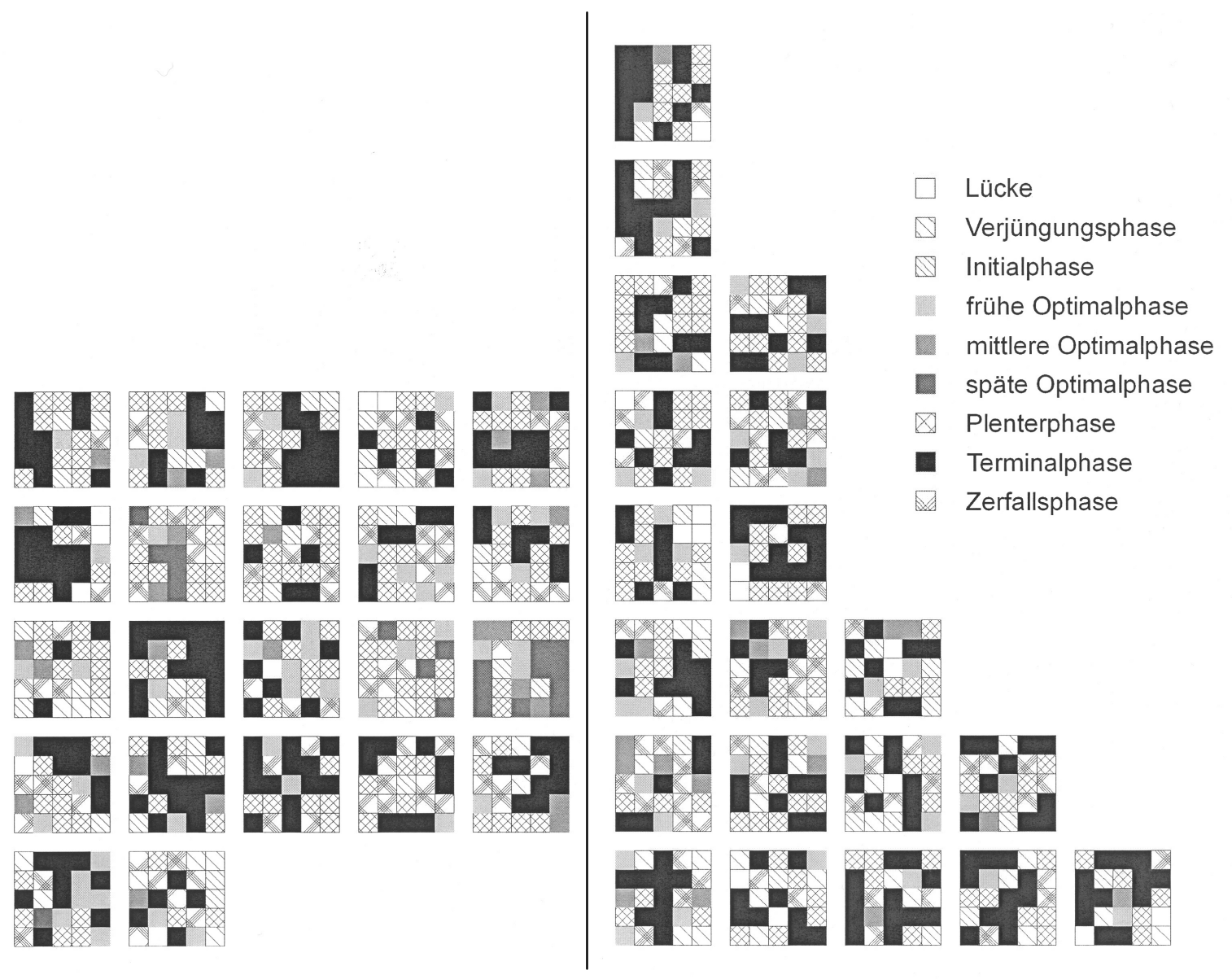

Abb. 27 Ausscheidung der Waldentwicklungsphasen auf 12,5 x 12,5 m-Flächen für die Urwaldreservate Havešová (links) und Kyjov (rechts). Die einzelnen Probeflächen sind in Havešová 200 m und in Kyjov 20 m voneinander entfernt.

Die Ausscheidung der Waldentwicklungsphasen auf relativ kleinen Rasterflächen (Abb. 27) veranschaulicht für Havešová, dass die älteren und häufigeren Entwicklungsphasen großflächiger auftreten können, als jüngere und seltenere Phasen. So nahmen einzelne geschlossene Flächen der Terminalphase in 7 Fällen mehr $1.000 \mathrm{~m}^{2}$ ein. Die Zerfallsphase nahm in 3 Fällen und die Plenterphase in 7 Fällen mehr als $600 \mathrm{~m}^{2}$ ein. Die begrenzte Probeflächengröße kann zu einer Zerschneidung größerer Flächeneinheiten führen. Relativiert man großflächiger auftretende Phasen entsprechend ihrer Anteile an der Waldfläche, zeigte sich für die Plenter- und Terminalphase immer noch eine Tendenz zum großflächigeren Auftreten. Die Zerfallsphase kam dagegen mit gleichgroßer Wahrscheinlichkeit auf einzelnen Rasterflächen vor wie die jüngeren Phasen. 
Tabelle 26 und 27 geben das mittlere Holzvolumen während unterschiedlicher Phasen der Waldentwicklung wieder. Insgesamt betrug das durchschnittliche Holzvolumen in Havešová $720 \mathrm{~m}^{3} / \mathrm{ha}$. Es wuchs in der mittleren Optimalphase auf $330 \mathrm{~m}^{3} /$ ha und betrug während der späten Optimal-, Zerfalls- und Plenterphase rund 1000 m³/ha. Das Totholzvolumen betrug im Durchschnitt $130 \mathrm{~m}^{3} / \mathrm{ha}$. Es stieg während der Zerfalls- und Verjüngungsphase auf mehr als $450 \mathrm{~m}^{3} /$ ha an.

In Kyjov betrug das durchschnittliche Holzvolumen der lebenden Bäume 450 m³/ha. Während der Plenter- und Terminalphase stieg es auf $640 \mathrm{~m}^{3} / \mathrm{ha}$ an. Das Totholzvolumen betrug durchschnittlich 110 m³/ha. Es war auch hier während der Zerfallsphase und den jüngeren Phasen deutlich höher als in der Optimal- oder Terminalphase.

Tab. 26 Statistische Kennwerte des Holzvorrats [ $\mathrm{m}^{3} / \mathrm{ha}$ ] lebender Bäume und des Totholzvorrates während unterschiedlicher Waldentwicklungsphasen in Havešová.

\begin{tabular}{llccccc}
\hline Phase & & $\begin{array}{c}\text { Mittel- } \\
\text { wert } \\
{\left[\mathrm{m}^{3} \text { ha-1] }\right.}\end{array}$ & $\begin{array}{c}\text { Standard- } \\
\text { abweichung } \\
{\left[\mathrm{m}^{3} \text { ha-1] }\right.}\end{array}$ & $\begin{array}{c}\text { unteres } \\
\text { Quartil } \\
{\left[\mathrm{m}^{3} \text { ha-1 }\right]}\end{array}$ & $\begin{array}{c}\text { oberes } \\
\text { Quartil } \\
{\left[\mathrm{m}^{3} \text { ha-1 }\right]}\end{array}$ & Fälle \\
\hline Lücke & leb. & 9 & 11 & 0 & 22 & 8 \\
& tot & 131 & 234 & 5 & 124 & 8 \\
Verjüngungs- leb. & 305 & 218 & 165 & 422 & 14 \\
phase & tot & 492 & 424 & 263 & 602 & 14 \\
Initial- & leb. & 39 & 61 & 7 & 36 & 34 \\
phase & tot & 200 & 246 & 0 & 349 & 34 \\
frühe & leb. & 214 & 374 & 72 & 152 & 49 \\
Optimalph. & tot & 14 & 75 & 0 & 0 & 49 \\
mittlere & leb. & 334 & 177 & 202 & 452 & 26 \\
Optimalph. & tot & 16 & 29 & 0 & 26 & 26 \\
späte & leb. & 1063 & 545 & 676 & 1330 & 20 \\
Optimalph. & tot & 24 & 72 & 0 & 19 & 20 \\
Plenter- & leb. & 954 & 556 & 564 & 1261 & 149 \\
phase & tot & 47 & 111 & 0 & 35 & 149 \\
Terminal- & leb. & 948 & 527 & 546 & 1251 & 163 \\
phase & tot & 42 & 84 & 0 & 46 & 163 \\
Zerfalls- & leb. & 428 & 422 & 108 & 606 & 87 \\
phase & tot & 447 & 267 & 232 & 595 & 87 \\
\hline & & & & & &
\end{tabular}


Tab. 27 Statistische Kennwerte des Holzvorrats [m³/ha] lebender Bäume und des Totholzvorrates während unterschiedlicher Waldentwicklungsphasen in Kyjov.

\begin{tabular}{llccccc}
\hline Phase & & $\begin{array}{c}\text { Mittel- } \\
\text { wert } \\
{\left[\mathrm{m}^{3} \text { ha-1] }\right.}\end{array}$ & $\begin{array}{c}\text { Standard- } \\
\text { abweichung } \\
{\left[\mathrm{m}^{3} \text { ha-1] }\right.}\end{array}$ & $\begin{array}{c}\text { unteres } \\
\text { Quartil } \\
{\left[\mathrm{m}^{3} \text { ha-1] }\right.}\end{array}$ & $\begin{array}{c}\text { oberes } \\
\text { Quartil } \\
{\left[\mathrm{m}^{3} \text { ha-1] }\right.}\end{array}$ & Fälle \\
\hline Lücke & leb. & 7 & 9 & 0 & 13 & 14 \\
& tot & 144 & 127 & 26 & 198 & 14 \\
Verjüngungs- & leb. & 236 & 271 & 38 & 429 & 34 \\
phase & tot & 301 & 188 & 147 & 410 & 34 \\
Initial- & leb. & 25 & 17 & 13 & 32 & 23 \\
phase & tot & 134 & 175 & 0 & 218 & 23 \\
frühe & leb. & 131 & 81 & 77 & 166 & 41 \\
Optimalph. & tot & 7 & 20 & 0 & 0 & 41 \\
mittlere & leb. & 247 & 91 & 173 & 336 & 20 \\
Optimalph. & tot & 26 & 33 & 0 & 47 & 20 \\
späte & leb. & & & & & 0 \\
Optimalph. & tot & & & & & \\
Plenter- & leb. & 677 & 317 & 422 & 838 & 119 \\
phase & tot & 21 & 41 & 0 & 19 & 119 \\
Terminal- & leb. & 607 & 268 & 397 & 755 & 183 \\
phase & tot & 31 & 61 & 0 & 26 & 183 \\
Zerfalls- & leb. & 230 & 187 & 77 & 314 & 66 \\
phase & tot & 318 & 221 & 186 & 397 & 66 \\
\hline
\end{tabular}

Tab. 28 Statistische Kennwerte der Stammzahl, Maximalhöhe und Summe der Überschirmung während unterschiedlicher Waldentwicklungsphasen in Havešová und Kyjov.

\begin{tabular}{|c|c|c|c|c|c|c|c|c|c|}
\hline \multirow[b]{2}{*}{ Reservat } & \multirow[b]{2}{*}{ Phase } & \multirow[b]{2}{*}{ Fälle } & \multicolumn{3}{|c|}{ Stammzahl [N/ha] } & \multicolumn{2}{|c|}{ Maximalhöhe [m] } & \multicolumn{2}{|c|}{ Überschirmung [\%] } \\
\hline & & & Mittel & Stdabw. & Max & Mittel & Stdabw. & Mittel & Stdabw \\
\hline \multirow[t]{9}{*}{ Havešová } & Lücke & 8 & 26 & 34 & 64 & 7,3 & 10,9 & 9 & 12,7 \\
\hline & Verjüngungsph. & 14 & 205 & 118 & 448 & 37,5 & 8,2 & 132 & 70,6 \\
\hline & Initialphase & 34 & 307 & 150 & 768 & 19,7 & 4,1 & 94 & 44,4 \\
\hline & frühe Optimalph. & 49 & 301 & 129 & 576 & 31,5 & 5,5 & 126 & 47,1 \\
\hline & mittlere Optimalph. & 26 & 269 & 144 & 576 & 35,3 & 10,4 & 157 & 73,2 \\
\hline & späte Optimalph. & 20 & 262 & 132 & 576 & 44,9 & 2,3 & 260 & 97,8 \\
\hline & Plenterphase & 149 & 365 & 116 & 896 & 43,9 & 2,4 & 284 & 86,1 \\
\hline & Terminalphase & 163 & 250 & 131 & 1088 & 43,7 & 2,2 & 243 & 100,1 \\
\hline & Zerfallsphase & 87 & 275 & 141 & 640 & 37,3 & 5,8 & 152 & 74,3 \\
\hline \multirow[t]{9}{*}{ Kyjov } & Lücke & 14 & 38 & 49 & 128 & 7,5 & 9,2 & 11 & 13,0 \\
\hline & Verjüngungsph. & 34 & 224 & 165 & 640 & 22,7 & 9,6 & 115 & 85,6 \\
\hline & Initialphase & 23 & 262 & 101 & 512 & 17,0 & 1,6 & 66 & 27,7 \\
\hline & frühe Optimalph. & 41 & 390 & 188 & 896 & 23,3 & 1,9 & 138 & 64,2 \\
\hline & mittlere Optimalph. & 20 & 333 & 146 & 576 & 26,7 & 0,5 & 161 & 60,9 \\
\hline & späte Optimalph. & 0 & & & & & & & \\
\hline & Plenterphase & 119 & 397 & 122 & 768 & 30,7 & 1,9 & 254 & 77,9 \\
\hline & Terminalphase & 183 & 301 & 171 & 896 & 30,9 & 1,7 & 215 & 73,8 \\
\hline & Zerfallsphase & 66 & 307 & 156 & 768 & 25,7 & 3,7 & 138 & 55,4 \\
\hline
\end{tabular}


Die Stammzahl betrug im Durchschnitt 290 Bäume/ha in Havešová und 320 Bäume/ha in Kyjov. Während in der Lückenphase kaum Bäume vorkamen, war die Dichte in der Plenterphase am höchsten und erreichte fast 400 Bäume/ha (Tabelle 28). Die Initialphase lag mit etwa 300 Bäumen deutlich darunter. Auch die Maximalwerte waren niedriger. Die Überschirmung (hier die Summe der überschirmten Fläche einzelner Bäume) war in der Plenterphase entsprechend hoch. Die Summe der Schirmfläche überstieg die Bezugsfläche um das Zweieinhalbfache (250 \%). Die durchschnittliche Überschirmung betrug in Havešová 210 \% und in Kyjov 186 \% der Waldfläche über alle Phasen. Die Oberhöhe der Bestände betrug $45 \mathrm{~m}$ in Havešová und $30 \mathrm{~m}$ in Kyjov (siehe 2.3.6.3). Die maximale Höhe der Bäume nahm mit Ausnahme der Verjüngungsphase in älteren Phasen zu.

Tab. 29 Verbale Beschreibung der Inital- und Optimalphase durch Tabaku (1999)

\begin{tabular}{|c|c|}
\hline Lücke & $\begin{array}{l}\text { Die Überschirmung des Derbholzbestandes liegt unter } 30 \% \text { und der Anteil der Verjüngungs- } \\
\text { flächen (Jungwuchs und Dickung) unter } 50 \% \text {. }\end{array}$ \\
\hline $\begin{array}{l}\text { Verjüngungs- } \\
\text { phase }\end{array}$ & $\begin{array}{l}\text { Bei langsamer Auflösung des Altbestandes entsteht in Lücken eine Verjüngungsphase mit } \\
\text { gruppen- und horstweise verteilten jungen Bäumen unter } 7 \mathrm{~cm} \text { BHD (Jungwuchs und Dickung) } \\
\text { mit einer Deckung von mehr als } 50 \% \text { der Bezugsfläche. }\end{array}$ \\
\hline Initialphase & $\begin{array}{l}\text { Stammzahlreiche Jungbestände unter } 20 \mathrm{~cm} \text { BHD bis Stangenholz, hervorgegangen aus } \\
\text { Naturverjüngung. Diese Phase kann nicht der Optimalphase zugeordnet werden, weil ihre } \\
\text { Holzvorräte erheblich geringer sind. Die Phase ist bereits leicht vertikal gestuft und besitzt } \\
\text { eine hohe Stammzahl. }\end{array}$ \\
\hline $\begin{array}{l}\text { Frühe } \\
\text { Optimalphase } \\
\text { Mittlere } \\
\text { Optimalphase }\end{array}$ & $\begin{array}{l}\text { Beginnend mit dem Erreichen eines BHD von } 20 \mathrm{~cm} \text { zeichnet sich die Optimalphase durch } \\
\text { einen deutlichen Nettoaufbau an Holzbiomasse aus. Es sind stammzahlreiche und zuwachs- } \\
\text { kräftige Baumholzbestände mit vitaler Oberschicht. Die Baumzahlen der Mittel- und Ober- } \\
\text { schicht nehmen mehr oder weniger ab. Kronendachlücken schließen sich rasch. Jungwuchs } \\
\text { fehlt fast ganz. }\end{array}$ \\
\hline $\begin{array}{l}\text { Späte } \\
\text { Optimalphase }\end{array}$ & $\begin{array}{l}\text { Die Optimalphase wird nach dem maximalen BHD in drei weitere Teilphasen unterteilt. Frühe } \\
\text { Optimalphase bei } 20-40 \mathrm{~cm} \text {, mittlere bei } 41-60 \text { und späte bei BHD über } 60 \mathrm{~cm} \text {. }\end{array}$ \\
\hline $\begin{array}{l}\text { Plenter- } \\
\text { phase }\end{array}$ & $\begin{array}{l}\text { Große Durchmesserdifferenzierung auf kleiner Fläche. Da sich auf- und abbauende Prozesse } \\
\text { gegenseitig ausgleichen, findet kein Nettoaufbau an Derbholzbiomasse statt. Es besteht eine } \\
\text { Entwicklungstendenz zur Optimalphase. Diese Struktur kann vorübergehend auch durch } \\
\text { einen sehr langen Verjüngungszeitraum oder durch die räumliche Überlagerung von Zerfalls- } \\
\text { und früher Optimalphase entstehen. }\end{array}$ \\
\hline Terminalphase & $\begin{array}{l}\text { Starke Baumholzbestände mit altersbedingtem Ausfall einzelner Bäume und Trupps. Es } \\
\text { findet kein Nettoaufbau an Derbholzbiomasse statt, weil der Zuwachs altersbedingt stagniert. } \\
\text { Stammzahlärmere Starkholzbestände mit Vorratsmaximum, Vitaliätsrückgang und } \\
\text { beginnender Bestandesauflösung. Zunehmende Mortalität in der Oberschicht. Je nach } \\
\text { Fortschritt der Vorratsabnahme geht die Terminalphase in die Zerfallssphase über. }\end{array}$ \\
\hline $\begin{array}{l}\text { Zerfalls- } \\
\text { phase }\end{array}$ & $\begin{array}{l}\text { Sehr stammzahlarme, stärker aufgelockerte Starkholz-Restbestände mit raschem Vorrats- } \\
\text { abbau und beginnender Verjüngung. Hoher Totholzvorrat bei zunehmender vertikaler } \\
\text { Strukturierung und größere Bestandeslücken. Falls sich auf dem überwiegenden Teil der } \\
\text { Fläche Verjüngung etabliert hat, geht die Zerfallsphase in die Verjüngungsphase über. }\end{array}$ \\
\hline
\end{tabular}




\subsubsection{Die Gruppierung von Bäumen unterschiedlicher Alterstufen}

Das Verfahren der Phasenausscheidung von Tabaku (1999) definiert die Initial- und

Optimalphase in erster Linie über den BHD der Bäume in einem Rasterfeld (Tab. 29). Diese Ausscheidung der Phasen lässt sich deshalb gut durch ein zweites Verfahren überprüfen, dass einem Ansatz von Grassi et al. (2003) folgt. Dabei werden nur Bäume einer

Durchmesserklasse und die Lage dieser Bäume analysiert. Die Nachbarschaft von Bäumen wird untersucht, ohne sich vorher auf Rasterfelder festzulegen. Gruppen von Bäumen einer Durchmesserklasse werden ausgeschieden, wenn sich die Kronen der Bäume berühren.

Tab. 30 Urwaldreservat Havešová: Häufigkeiten von Gruppen benachbarter Bäumen einer Durchmesserklasse, hochgerechnet auf 10 ha. Gruppierungskriterium ist Kronenkontakt.

\begin{tabular}{|c|c|c|c|c|}
\hline \multirow[b]{2}{*}{$\begin{array}{c}\text { Anzahl der } \\
\text { Bäume je Gruppe }\end{array}$} & \multicolumn{4}{|c|}{ Durchmesserklasse/Entwicklungsphase } \\
\hline & $\begin{array}{l}\text { 7-20 cm } \\
\text { Initial- } \\
\text { phase }\end{array}$ & $\begin{array}{c}21-40 \mathrm{~cm} \\
\text { frühe } \\
\text { Optimalphase }\end{array}$ & $\begin{array}{c}41-60 \mathrm{~cm} \\
\text { mittlere } \\
\text { Optimalphase } \\
\end{array}$ & $\begin{array}{c}61-100 \mathrm{~cm} \\
\text { späte } \\
\text { Optimalphase }\end{array}$ \\
\hline 1 & 230.4 & 173.4 & 101.2 & 41.9 \\
\hline 2 & 96.6 & 67.5 & 25.6 & 15.1 \\
\hline 3 & 40.7 & 18.6 & 18.6 & 5.8 \\
\hline 4 & 20.9 & 14.0 & 7.0 & 7.0 \\
\hline 5 & 20.9 & 4.7 & 3.5 & 5.8 \\
\hline 6 & 10.5 & 3.5 & 3.5 & 2.3 \\
\hline 7 & 10.5 & 2.3 & 1.2 & 4.7 \\
\hline 8 & 7.0 & & 1.2 & 4.7 \\
\hline 9 & 4.7 & 1.2 & & 2.3 \\
\hline 10 & 2.3 & 1.2 & 1.2 & \\
\hline 11 & 3.5 & & 1.2 & 2.3 \\
\hline 12 & 1.2 & 1.2 & 1.2 & 2.3 \\
\hline 13 & 2.3 & & & \\
\hline 14 & 1.2 & & & 1.2 \\
\hline 15 & 1.2 & & & \\
\hline 16 & & & & 1.2 \\
\hline 17 & 1.2 & & & \\
\hline 18 & & & & \\
\hline 19 & 1.2 & & & 1.2 \\
\hline 20 & 1.2 & & & \\
\hline 21 & 1.2 & & & 1.2 \\
\hline 22 & & & & \\
\hline 23 & 1.2 & & & \\
\hline 24 & & & & \\
\hline 25 & & & & \\
\hline 26 & & & & \\
\hline 27 & & & & \\
\hline 28 & 1.2 & & 1.2 & \\
\hline 29 & 1.2 & & & \\
\hline 30 & & & & \\
\hline 31 & & & & \\
\hline 32 & & & & \\
\hline 33 & & & & \\
\hline 34 & 1.2 & & & \\
\hline 35 & & & & \\
\hline 36 & & & & \\
\hline 37 & & & & \\
\hline 38 & 1.2 & & & \\
\hline 39 & & & & \\
\hline 40 & & & & \\
\hline 42 & 1.2 & & & \\
\hline 47 & 1.2 & & & \\
\hline 64 & 1.2 & & & \\
\hline 67 & 1.2 & & & \\
\hline $\begin{array}{l}\text { Anzahl der Bäume } \\
\text { je Durchmesserklasse: }\end{array}$ & 1590.7 & 516.7 & 363.1 & 386.3 \\
\hline
\end{tabular}


Tab. 31 Urwaldreservat Kyjov: Häufigkeiten von Gruppen benachbarter Bäumen einer Durchmesserklasse, hochgerechnet auf 10 ha. Gruppierungskriterium ist Kronenkontakt der Bäume.

\begin{tabular}{|c|c|c|c|c|}
\hline \multirow[b]{2}{*}{$\begin{array}{c}\text { Anzahl der } \\
\text { Bäume je Gruppe }\end{array}$} & \multicolumn{4}{|c|}{ Durchmesserklasse/Entwicklungsphase } \\
\hline & $\begin{array}{l}\text { 7-20 cm } \\
\text { Initial- } \\
\text { phase }\end{array}$ & $\begin{array}{c}21-40 \mathrm{~cm} \\
\text { frühe } \\
\text { Optimalphase }\end{array}$ & $\begin{array}{c}41-60 \mathrm{~cm} \\
\text { mittlere } \\
\text { Optimalphase }\end{array}$ & $\begin{array}{c}61-100 \mathrm{~cm} \\
\text { späte } \\
\text { Optimalphase }\end{array}$ \\
\hline 1 & 268.8 & 158.7 & 99.8 & 57.6 \\
\hline 2 & 93.4 & 65.3 & 25.6 & 24.3 \\
\hline 3 & 53.8 & 24.3 & 12.8 & 10.2 \\
\hline 4 & 34.6 & 11.5 & 15.4 & 3.8 \\
\hline 5 & 16.6 & 9.0 & 7.7 & 2.6 \\
\hline 6 & 9.0 & 3.8 & & 2.6 \\
\hline 7 & 7.7 & 2.6 & 5.1 & 1.3 \\
\hline 8 & 7.7 & 2.6 & & 3.8 \\
\hline 9 & 11.5 & 1.3 & & 2.6 \\
\hline 10 & 6.4 & 1.3 & 2.6 & 1.3 \\
\hline 11 & 5.1 & 1.3 & 1.3 & 1.3 \\
\hline 12 & 3.8 & & & \\
\hline 13 & 2.6 & & & 1.3 \\
\hline 14 & 1.3 & 1.3 & & 1.3 \\
\hline 15 & 1.3 & 1.3 & & 1.3 \\
\hline 16 & 2.6 & 1.3 & & \\
\hline 17 & 2.6 & & & \\
\hline 18 & 2.6 & & & \\
\hline 19 & & 1.3 & & 1.3 \\
\hline 20 & 1.3 & & & 1.3 \\
\hline 21 & 1.3 & & & \\
\hline 22 & & & & 1.3 \\
\hline 23 & & & & \\
\hline 24 & & & & \\
\hline 25 & 1.3 & & & \\
\hline 26 & & & & \\
\hline 27 & 1.3 & & & \\
\hline 28 & 1.3 & & & \\
\hline 29 & & & & \\
\hline 30 & 1.3 & & & \\
\hline 31 & & & & \\
\hline 32 & & & & \\
\hline 33 & & & & \\
\hline 34 & & & & \\
\hline 35 & & & & \\
\hline 36 & & & & \\
\hline 37 & & & & \\
\hline 38 & 1.3 & & & \\
\hline 39 & & & & \\
\hline 40 & & & & \\
\hline 41 & 1.3 & & & \\
\hline $\begin{array}{l}\text { Anzahl der Bäume } \\
\text { je Durchmesserklasse: }\end{array}$ & 1795.8 & 634.9 & 364.8 & 401.9 \\
\hline
\end{tabular}

Tabelle 30 und 31 zeigten sehr ähnliche Häufigkeiten von unterschiedlich großen Gruppen in beiden Reservaten. Die Gruppenhäufigkeiten der späten Optimalphase ähnelten sich, obwohl nach dem Verfahren von Tabaku (1999) in Kyjov gar keine späte Optimalphase ausgeschieden wurde. 


\subsection{Diskussion}

Die Anteile der Waldentwicklungsphasen sind gut mit Ergebnissen aus albanischen Urwäldern vergleichbar. Drei von Tabaku (1999) untersuchte Urwaldbestände in Albanien ergaben für die Lücken- und Verjüngungsphase gleichgroße Anteile wie in Havešová. Die Anteile der Lückenphase schwankten in allen Beständen um $2 \%$. Die Initialphase nahm in den albanischen Beständen weniger Fläche ein als in den slowakischen. Die mittlere Optimalphase war deutlich stärker vertreten. Eine späte Optimalphase wurde sowohl in Albanien als auch in Kyjov nicht ausgewiesen. Der Anteil der Terminalphase war im Vergleich zu Havešová und Kyjov 50 \% höher, der Anteil der Zerfallsphase war dagegen um 50-80\% niedriger.

Wie bereits Tabaku (1999) hervorhob, besteht ein entscheidender Unterschied zu hiebsreifen Wirtschaftswäldern im Vorhandensein und der Dominanz der Terminalphase sowie einem drei- bis fünfmal geringerem Anteil der Optimalphase. Für weitergehende Vergleiche mit Wirtschaftswäldern und Naturwaldreservaten verweisen wir hier auf die Arbeit von Tabaku (1999). Andere Naturwaldreservate wurden in Niedersachsen von Meyer (1999) untersucht und Entwicklungsphasen ausgeschieden. In einigen Reservaten konnte bereits eine Terminaloder Zerfallsphase ausgewiesen werden, allerdings nahm ihr Flächenanteil nur wenige Prozente ein. Der Anteil der Optimalphase lag in den meisten Reservaten bei $90 \%$. Im Vergleich zu einem Optimalphasenanteil von $15 \%$ im Urwald kann man später noch stärkere Anteile der Terminal- und Zerfallsphase erwarten. Allerdings sind die Buchen in diesen Reservaten maximal 170 Jahre alt und können noch weitere 100 Jahre leben. Schätzungen zur Dauer eines Waldzyklus (turnover time) in Buchenwäldern bewegen sich zwischen 220-250 (Korpel 1967, 1995) und 250-300 Jahren (Chernyavskyy 2005). In einem einaltrigen Bestand Dänemarks vermutete Emborg et al. (2000) eine Dauer von 284 Jahren.

Großflächigere Auflösungserscheinungen mit zunehmendem Alter sind in den Naturwaldreservaten möglich. So zeigt das Naturwaldreservat Heilige Hallen mit einem 250jährigen Bestand einen Anteil der Lückenphase von 26,5 \% (Tabaku 1999). Von anderen Naturwaldreservaten (120-160 Jahre alte Bestände) wird dagegen über eine Neubildung von Lücken auf nur 0,15 \% der Fläche im Jahr berichtet (Richter 1990). In den slowakischen Buchenurwäldern liegt die Lückenbildungsrate bei ungefähr 0,4 \% im Jahr (Drößler und Lüpke 2005).

Mit abnehmender Vitalität der Bäume ist deshalb in einigen Naturwaldreservaten eine verstärkte Lückenbildung zu erwarten. 
Die kleinflächige Darstellung der Waldentwicklungsphasen (Abb. 26) bestätigte ein häufigeres zusammenhängendes Auftreten der dominanten älteren Phasen in Urwäldern, wie auch Tabaku (1999) zeigen konnte.

Der Aufbau des Holzvorrats verlief ähnlich wie in albanischen Urwäldern. Er lag in der Lückenphase nahezu bei Null und erreicht sein Maximum in der Plenterphase oder späten Optimalphase. Der maximale Holzvorrat betrug dann je nach Höhenwachstum der Bäume 650 bis $1100 \mathrm{~m}^{3} / \mathrm{ha}$. Das Totholzvolumen war in allen Urwaldbeständen während der Zerfalls-, Lücken-, Verjüngungs- und Initialphase am höchsten. Es betrug während der Zerfalls- und Verjüngungsphase $400 \mathrm{~m}^{3} / \mathrm{ha}$. In der Optimal-, Plenter- und Terminalphase lag es unter 50 $\mathrm{m}^{3} / \mathrm{ha}$.

Ähnlich Werte ergaben sich jeweils für die Holz- und Totholzvorräte in der Zerfalls- und Verjüngungsphase. Während die Ausscheidungskriterien eine klare Zweiteilung der Phasen vornahm, gingen diese in Wirklichkeit oft fließend ineinander über.

Das Verfahren von Tabaku (1999) unterscheidet die Phasen anhand einfach abzuleitender Hilfsindikatoren. Sie entsprechen Werten, die zum Teil von Korpel' (1995) für bestimmte Entwicklungsphasen abgeleitet worden sind. Einen Kritikpunkt an diesem Ausscheidungsverfahren stellen die maximalen Durchmesser als Schwellenwerte für die Optimalphasen und der Totholzanteil als Kriterium für die Zerfallsphase dar. Die Vorräte dieser Phasen können deshalb bestimmte Werte nicht unterschreiten.

Die Stammzahl erreichte in den verschiedenen Urwäldern sehr ähnliche Werte. Sie stieg von ungefähr 30 Bäumen/ha in der Lückenphase auf knapp 400 Bäume/ha in der frühen Optimalphase an. In der Plenterphase standen ebenfalls 400 Bäume auf einem Hektar, während sich die Zahl in der Zerfallsphase wieder auf maximal 300 Bäume/ha verringerte. Die relative Entwicklung der Maximalhöhen verlief ebenfalls identisch. Bis auf die Verjüngungsphase, in der auch noch sehr hohe Bäume vorkamen, stieg die Höhe in albanischen Reservaten ab der Lückenphase kontinuierlich an und erreichte ihr Maximum in der Plenterphase. Die Summe der Überschirmung erreichte ihr Maximum in der Plenterphase mit durchschnittlich $290 \%$. Über alle Phasen gemittelt lag die Summe der Überschirmung in den albanischen Reservaten bei 207 bis 266 \% (Tabaku 1999).

Als Fazit aus dem Vergleich der 5 Urwaldbestände ergab sich, dass die ausgeschiedenen Phasen den Zustand der unterschiedlich fortgeschrittenen Waldentwicklung gut und plausibel wiedergeben konnten und sich die Ergebnisse leicht zusammenfassen lassen. Die 
Phasenausscheidung war sogar in der Lage, die im Urwald Kyjov vor 2-3 Jahrzehnten aufgetretenen stärkeren (anthropogenen) Störungen anzuzeigen, die sich auch in der Durchmesserverteilung des Bestandes wieder spiegelten (Drößler und Lüpke 2006a). Beträchtliche Unterschiede traten dagegen im Vergleich des Anteils der Lückenphase mit dem tatsächlichen Anteil der Lücken auf. Dem geringen Anteil der Lückenphase von etwa $2 \%$ stehen Ergebnisse einer Lückeninventur in Havešová und Kyjov entgegen (Drößler und Lüpke 2005). Bestandeslücken, die keine Bäume mit einem BHD $\geq 7 \mathrm{~cm}$ aufweisen, wurden direkt angesprochen und erreichen einen Anteil von 7 bzw. $8 \%$ an der Waldfläche. Erklären lassen sich die unterschiedlichen Ergebnisse durch den hohen Anteil an Mehrfachüberschirmungen im Bestand. Das Verfahren nach Tabaku (1999) summiert für jedes Rasterfeld die Überschirmung durch jeden Baum auf. Die durchschnittliche Überschirmung beträgt etwa $200 \%$. Wir nehmen an, dass der Lückenanteil dadurch um mehr als die Hälfte unterschätzt wurde. Ein anderer Grund für die unterschiedlichen Ergebnisse liegt an dem großen Anteil sehr kleiner Lücken. Nimmt die überschirmungsfreie Fläche auf einem Rasterfeld mehr als $30 \%$ des Rasterfeldes (47 m²) ein, würde keine Lücke ausgeschieden, weil die Summe der Überschirmung auf der restlichen Fläche des Rasterfeldes im Durchschnitt weit über $70 \%$ beträgt. Tatsächlich müsste die Lücke größer als $120 \mathrm{~m}^{2}$ sein, damit die durchschnittliche Summe der Überschirmung eines Rasterfeldes weniger als $70 \%$ beträgt. Lücken unter $120 \mathrm{~m}^{2}$ Größe nehmen in den slowakischen Urwäldern bis zu $1 / 5 \mathrm{der}$ Lückenfläche ein. Um die Schätzung zu verbessern, müsste die einfach überschirmte Fläche berechnet werden. Das Ziel der Untersuchungen von Tabaku (1999) war aber nicht die Herleitung des genauen Lückenanteils, sondern die kleinflächige Charakterisierung der Bestandestextur über den gesamten Entwicklungszyklus mit dem Standraum einer Altbuche als Mosaikbaustein. Die Ergebnisse unserer Untersuchung zeigen, dass Lückenanteile aus den verwendeten Hilfsindikatoren nicht abzuleiten sind.

Die Phasenausscheidung auf der Grundlage von Maximalwerten (maximaler BHD und maximale Höhe) führt dazu, dass kleinere Bäume älteren Phasen zugeordnet werden können. So setzt sich die Plenterphase aus kleinen und großen Bäumen zusammen. Bei der schwer überschaubaren Anordnung unterschiedlich starker Bäume im Urwald kann man aber auch den Standpunkt vertreten, dass der einzelne Baum die grundlegende Einheit zur Ausscheidung von Waldentwicklungsphasen darstellt. Grassi et al. (2003) hat aus diesem Standpunkt heraus eine Phasenausscheidung für bewirtschaftete ungleichaltrige Nadelbaumbestände entwickelt. In Abhängigkeit von der Höhe der Bäume weist er anhand der überschirmten Fläche 
Entwicklungsphasen aus. Diese Idee verfolgt auch die Gruppierung von Bäumen ähnlicher Größe. In Tabelle 30 und 31 wurde deutlich, dass Bäume einer bestimmten

Durchmesserklasse am häufigsten einzeln vorkamen und dass die Anzahl der Gruppen mit zunehmender Gruppengröße exponentiell abnahm. Ein Baum der Durchmesserklasse 7-20 cm hatte durchschnittlich weniger als $25 \mathrm{~m}^{2}$ Kronenschirmfläche. Ein Baum der Klasse 21-40 cm hatte durchschnittlich weniger als $50 \mathrm{~m}^{2}$. Einzeln stehende Bäume einer Klasse dominierten auch unabhängig von verschiedenen Klasseneinteilungen. Wie im Abschnitt 3.5.1 gezeigt wurde, kann diese exponentielle Häufigkeitsverteilung der Baumgruppen einen wichtigen Unterschied zwischen Urwald und Altersklassenwald darstellen.

Während das Verfahren von Tabaku (1999) neun Phasen unterscheidet, unterscheidet Korpel' (1995) drei Entwicklungsstadien: Das Stadium des Heranwachsens, das Optimalstadium und das Zerfallsstadium. Eine Gliederung des Optimalstadiums in drei Phasen erwies sich bisher in Urwäldern aufgrund des geringen Flächenanteils nicht als sinnvoll (Saniga 1999, 2002, 2003). Einzelne Phasen wurden gar nicht ausgeschieden. Um eine bessere Vergleichbarkeit der slowakischen und deutschen Forschungsergebnisse zu erzielen, kann man die Verjüngungs- und Initialphase zum Stadium des Heranwachsens und die Terminal-, Zerfallund Lückenphase zum Zerfallsstadium zusammenfassen.

Eine Schlüsselrolle bei der Waldentwicklung kommt den Bestandeslücken zu (Runkle 1985). Ihre Größe stellt die Fläche für einen neu beginnenden Waldzyklus dar. Wie im Abschnitt 4.5.2 gezeigt wurde, dominieren im Buchenurwald kleine Lücken, während die Anzahl der Lücken mit zunehmender Größe exponentiell stark abnimmt. Neben der Möglichkeit, dass sich benachbarte Randbäume auf die Lücken ausdehnen, werden auch junge Bäume in den Lücken aufwachsen. Dabei kommt es zu Überlappungen der Kronen, wie die Summe der Kronenschirmfläche von 189-266 \% beweist. Grassi et al. (2003) haben ebenfalls Baumkronen kartiert und stellen eine Überlagerung jüngerer Phasen durch ältere fest. Die einzelbaumweise Betrachtung ermöglicht detailierte Aussagen über die Entwicklung der lebenden Bäume und über die Flächengröße der Intialphase und Optimalphasen, während die Phasenausscheidungen nach Tabaku (1999) und Meyer (1999) einen Ansatz verfolgen, der alle Waldentwicklungsphasen erfasst. Die Phasen werden zum Teil durch die größten Bäume auf einer Rasterfläche definiert. Unter den Kronen dieser Bäume befinden sich oft andere Bäume, die für sich betrachtet die Verjüngungsphase durchlaufen oder bereits durchlaufen haben. Beim Ableben eines sehr großen Baumes wäre dadurch auch der unmittelbare Übergang zur Initial- oder Optimalphase möglich. Nachteil der einzelbaumweisen 
Betrachtung ist unter anderem die fehlende Berücksichtigung des Totholzes, womit die späten Phasen nicht erfasst werden können.

Beide Methoden der Bestandesanalyse ergänzen sich aber gut und können gegenseitig

Schwächen erkennen lassen. Wir sehen beide Methoden als wichtige Instrumente an, um die

Entwicklung des Waldaufbaus in Naturwäldern auf objektiver Grundlage zu beschreiben.

\subsection{Literatur}

Chernyavskyy, M. 2005. The dynamics of virgin beech forests in the Ukrainian Carpathians. In: Hamor, F.D.

and Commarmot, B. (eds): Natural forests in the temperate zone of Europe - Values and utilisation. International conference in Mukachevo, Ukraine. October 13-17, 2003. Rakhiv, Carpathian biosphere Reserve; Birmensdorf, Swiss Federal Research Institute WSL.

Drößler, L., Lüpke, B. v. 2005. Canopy gaps in two virgin beech forest reserves in Slovakia. Journal of Forest Science, 51, 446-457.

Drößler, L. und Lüpke, B. v. 2006a: Bestandesstruktur, Verjüngung und Wachstumsfaktoren in zwei slowakischen Buchenurwald-Reservaten. AFJZ (im Druck).

Drößler, L. und Lüpke, B. v. 2006b. Stand structure in virgin and managed beech forests. European Journal of Forest Research (in press).

Emborg, J., Christensen, M. und Heilmann-Clausen, J. 2000. The structural dynamics of Suserov Skov, a nearnatural temperate deciduous forest in Denmark. For. Ecol. Manage. 126: 173-189.

Grassi, G., Minotta, G., Giannini, R. und Bagnaresi, U. 2003. The structural dynamics of managed uneven-aged conifer stands in the Italian eastern Alps. Forest Ecology and Management 185: 225-237.

Knapp, H. D. und Jeschke, L. 1991. Naturwaldreservate und Naturwaldforschung in den ostdeutschen Bundesländern. Schriftenreihe Vegetationskunde 21:21-54.

Korpel', Š. (1967): Vývoj a veková štruktúra bukoveho pralesa na vihorlate. Biologia 22(4): 285-303. Slowakisch.

Korpel', Š. 1995. Die Urwälder der Westkarpaten. Stuttgart, Jena, New York, G. Fischer Verlag. Leibundgut, H. 1993. Europäische Urwälder. Haupt Verlag, Bern, Stuttgart, Wien. 260 S.

Meyer, P. 1999. Bestimmung der Waldentwicklungsphasen und der Texturdiversität in Naturwäldern. AFJZ 170: 203-211.

Neumann, M. 1979. Bestandesstruktur und Entwicklungsdynamik im Urwald Rothwald/NÖ und im Urwald Čorkova Uvala/Kroatien. Diss. Univ. f. Bodenkultur, Wien.

Petráš, R. und Pajtík, J. 1991. Sústava Česko-slovenských objemových tabuliek drevín. Lesnícky Časopis, 37 : 49-56. Slowakisch.

Runkle, J.R. 1985. Disturbance regimes in temperate forests. In: Pickett, S.T.A. and White, P.S. (eds.): The ecology of natural disturbance and patch dynamics, p. 17-33. Academic press, Orlando.

Saniga, M.1999. Struktúra, produkcne a regeneracne procesy Badinskeho pralesa. Journal of forest science 45:121-130. Slowakisch.

Saniga, M. 2002. Struktúra, produkcne a regeneracne procesy bukoveho pralesa Rozok. Ochrana prirody 21: 207-218. Slowakisch.

Saniga, M. 2003. Struktúra, produkcne a regeneracne procesy bukoveho pralesa v NPR Havesova. Ochrana prirody 22: 131-140. Slowakisch.

Tabaku, V. 1999. Struktur von Buchen-Urwäldern in Albanien im Vergleich mit deutschen BuchenNaturwaldreservaten und -Wirtschaftwäldern. Göttingen, Univ., Diss. 


\section{Weitere Ergebnisse}

\subsection{Paar- und Markenkorrelationsfunktionen}

\subsubsection{Theorie der Korrelationsfunktionen}

Die Berechnung und Interpretation von Paar- und Markenkorrelationsfunktionen wird von Stoyan und Stoyan (1994) im Detail erläutert. In unserem Fall beschreiben die Markenkorrelationsfunktionen das relative Produkt aus den Durchmessern von zwei Bäumen, die in einem bestimmten Abstand voneinander stehen. Wenn mehr als ein Baumpaar mit diesem Abstand auftritt, wird der Durchschnitt der Produkte gebildet.

Die Paarkorrelationsfunktionen beschreiben dagegen die relative Häufigkeit von Baumpaaren mir einem bestimmten Abstand zwischen den beiden Bäume. Sie können einen Trend zur gleichmäßigen, zufälligen oder geklumpten Verteilung der Bäume anzeigen (Abb. 28). Eine zufällige Verteilung liegt vor, wenn die Funktion den Wert 1 erreicht. Beginnt die Funktion mit niedrigeren Werten, liegt eine Tendenz zur regelmäßigen Verteilung vor. Beginnt die Funktion mit höheren Werten, kommen viele Bäume mit geringerem Abstand zum Nachbarbaum als der durchschnittliche Baumabstand des Bestandes vor (Klumpung). Zusätzlich zu konventionellen Paarkorrelationsfunktionen wurden Paarkorrelationsfunktionen berechnet, deren Funktionswerte mit dem Durchmesser der beiden Bäume multipliziert wurde (Abb. 29). Diese Funktionswerte können als Schätzung für die Grundfläche dienen, mit der man die Verteilung der Grundfläche bzw. des Holzvolumens untersuchen kann (Stoyan, mündl. Mitteilg.). Ein Vergleich der beiden Paarkorrelationsfunktionen lässt mögliche Aggregationen kleiner Bäume erkennen. Die Berechnung der Korrelationsfunktionen wurde freundlicherweise von Herrn Dietrich Stoyan durchgeführt.

In den Urwaldreservaten wurden für jede Stichprobenfläche eine Paar- und eine Markenkorrelationsfunktion berechnet. Aus dem Durchschnitt der einzelnen Funktionswerte wurde später eine einzelne Korrelationsfunktion je Reservat geschätzt. Dieses Verfahren der Zusammenfassung bei Stichproben mit mehreren, separaten räumlichen Mustern beschreiben Ohser u. Mücklich (2000).

Zusätzlich wurde der Index von Clark u. Evans (1954) mit der Randkorrektur von Donelly (1978) berechnet. Dieser Index beschreibt ebenfalls Muster der Baumverteilung und wird häufiger in der Literatur erwähnt als Korrelationsfunktionen (z.B. Nagel 2003, Gleichmar u. Gerold 1998).

Eine Beschreibung der betrachteten Bestände und deren Aufnahmemethodik finden sich in Tabelle 14 auf S. 40. 
6.1.2 Ergebnisse und Diskussion der Korrelationsfunktionen
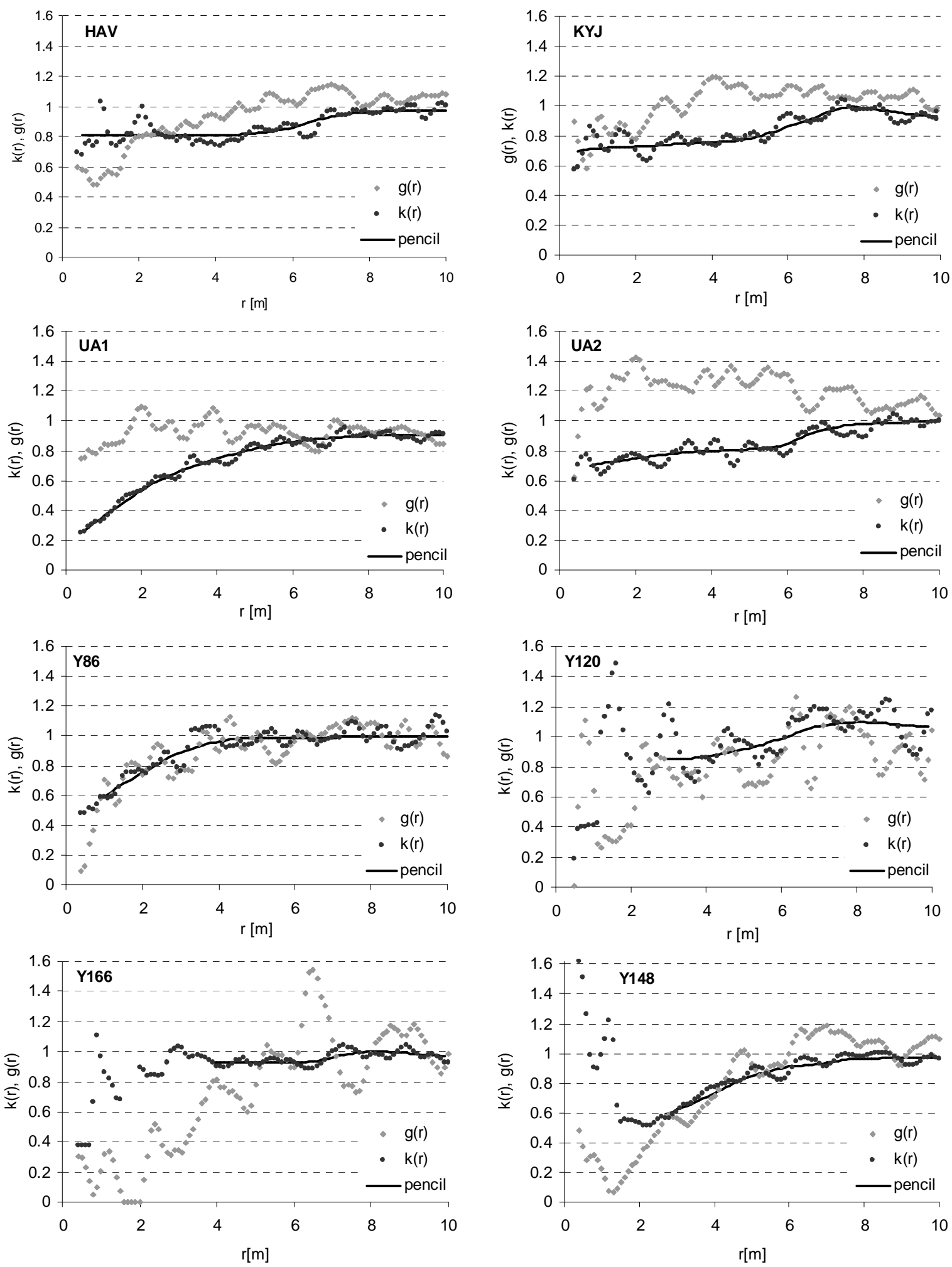

Abb. 28. Paarkorrelationsfunktionen g(r), Markenkorrelationsfunktionen k(r) und eine geglättete Trendlinie ,pencil“" von k(r). k(r) beschreibt Baumpaare in einer bestimmten Entfernung und stellt eine Beziehung her zwischen dem relativen, gemittelten Wert aus dem Produkt der BHD beider Bäume und der Entfernung $\mathrm{r}[\mathrm{m}]$. $\mathrm{g}(\mathrm{r})$ zeigt die relativen Häufigkeiten der Baumpaare. 
Die Markenkorrelationsfunktionen demonstrieren in beiden Urwäldern (HAV und KYJ in Abbildung 28) für Baumpaare mit weniger als 6-7 m Abstand kleinere Bäume als im Bestandesdurchschnitt. Die Funktionen hoben keine Unterschiede zwischen Urwäldern und dem Plenterwald UA2 hervor. Für den Plenterwald UA1 ergab sich ein schnellerer Anstieg der k(r)-Werte für sehr kleine Abstände, der aber graduell abnahm. Zu einem ähnlichen Ergebnis kamen Pommerening et al. (2000) in einem Tannen-Fichten-Buchen-Plenterwald. Die Funktion im einaltrigen Bestand Y86 zeigt einen ähnlichen Verlauf.

Der k(r)-Wert 1 entspricht dem durchschnittlichen Produkt der Durchmesser eines Baumpaares im Bestand und kann als zufällige Verteilung der Durchmesser interpretiert werden. Diesen Wert erreicht Bestand Y86 für Baumabstände größer als 3 m, Y120 für Abstände größer als 5 m und Y148 für Abstände größer als 6-7 m. Der Abstand in Y148 entspricht den Werten aus den Urwäldern. Eine schwache Beziehung zwischen dem Abstand und der Größe von Bäumen wurde im Bestand Y166 gefunden. Dieser Bestand setzte sich aus starken Bäumen mit geringer Durchmesserdifferenzierung zusammen.

Die Paarkorrelationsfunktionen zeigten eine zufällige Verteilung der Bäume in Urwäldern mit einer Tendenz zu regelmäßigen Mustern für Baumabstände unter $4 \mathrm{~m}$. Alte einaltrige Bestände zeigten eine regelmäßige Verteilung der Bäume für Abstände bis zu 6 m. In Plenterwäldern wurde eine zufälligere Baumverteilung mit einer Tendenz zur Klumpung beobachtet. Diese Klumpung wurde durch die Aggregation kleiner Bäume verursacht, wie sich durch die multiplikativ gewichtete Paarkorrelationsfunktion in Abbildung 29 feststellen ließ. Im Gegensatz zu den zufällig verteilten Bäumen war die Grundfläche regelmäßig verteilt für Baumabstände bis zu $6 \mathrm{~m}$. Die relativen Werte der mit zwei Durchmessern multiplikativ gewichteten Paarkorrelationsfunktion entsprechen im Verhältnis der Grundfläche. Für den Plenterwald UA1 und den Bestand Y166 ergab sich eine regelmäßig verteilte Grundfläche (Abb. 29). Für Y166 war die gewichtete Funktion der konventionellen Paarkorrelationsfunktion sehr ähnlich aufgrund der geringen Streuung der Durchmesser des Bestandes (siehe Abb. 14). Havešová zeigte eine zufällige Verteilung sowohl der Bäume als auch der Grundfläche. Beide Funktionen verliefen für Abstände unter $2 \mathrm{~m}$ und über $7 \mathrm{~m}$ ähnlich. Dazwischen zeigte sich eine im Vergleich zur Grundfläche zufälligere Verteilung der Bäume. Diese Ergebnisse führen zu dem Schluss, dass die Verteilung der Grundfläche bzw. des Holzvolumens für die computergestützte baumweise Generierung von ungleichaltrigen Beständen stärker zu berücksichtigen ist. Waldwachstumsmodelle wie SILVA nutzen bereits konventionelle Paarkorrelationsfunktionen (Pretzsch 2001). 

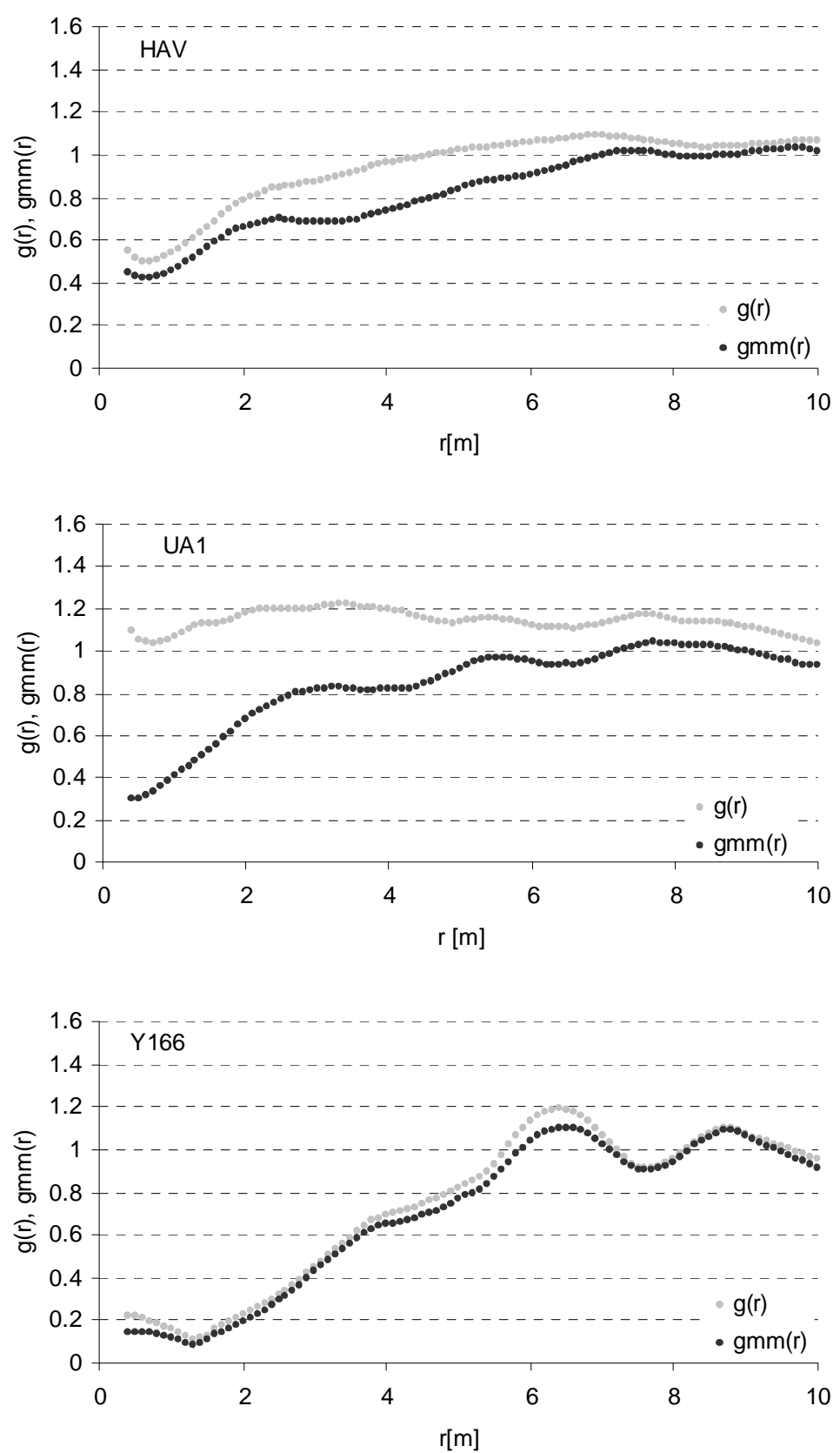

Abb. 29. Paarkorrelationsfunktionen gmm(r), multiplikativ gewichtet mit dem BHD der Bäume eines Paares, im Vergleich mit konventionellen Paarkorrelationsfunktionen g(r).

Die berechneten Clark-Evans (1954) Index-Werte schwankten von 0,98 im Bestand UA2 bis 1,23 in Y148. In den Urwäldern betrug der Durchschnittswert 1,1. Nimmt der Index den Wert 1 an, so liegt eine zufällige Verteilung vor. Höhere Werte deuten auf ein regelmäßiges Muster hin, wobei mit einem Wert 2,1491 ein streng hexagonales Muster gegeben ist. Werte unter 1 zeigen geklumpte Verteilungen an. Die berechneten Werte führten damit zu ähnlichen Ergebnissen wie die Paarkorrelationsfunktionen. 


\section{Abschließende Diskussion}

\subsection{Diskussion der Hypothesen}

(I) Die Struktur ist auf kleiner Fläche sehr heterogen. Die Bäume sterben aber nicht nur einzeln ab. - Beide Teile der Hypothese wurden bestätigt, obwohl sie auf den ersten Blick als Gegensatz erscheinen. Einzelbaumstörungen dominieren jedoch, wogegen großflächige Störungen sehr selten sind.

So bestanden $65 \%$ der Lücken aus jeweils einem einzelnen abgestorbenen Baum. Weitere 20 $\%$ der Lücken entstanden durch den Tod von maximal vier abgestorbenen Bäumen und tragen noch einen kleinflächig heterogenen Charakter. Größere Lücken (ab $600 \mathrm{~m}^{2}$ Fläche) können aber bis zur Hälfte der Gesamtlückenfläche einnehmen. In sehr seltenen Fällen treten Löcher von $1 / 2$ ha Größe auf (<1\% der Lücken). So wurde in beiden Reservaten eine maximale Kronendachöffnung von 0,4 ha festgestellt. Die Fläche bis zu den Stammfüßen der Lückenrandbäume betrug sogar 0,6 bzw. 0,7 ha. Die Extremwerte sind jedoch stark abhängig vom Stichprobenumfang. Die Vollaufnahme großer Bestandesflächen in albanischen Buchenurwäldern von Tabaku u. Meyer (1999) ergab nur kleine Lücken. Die größte Lücke, die Zeibig et al. (2005) in einem slowenischen Buchenurwald vorfanden, betrug $850 \mathrm{~m}^{2}$. Eine Luftbildinventur der größeren Urwaldgebiete in Rumänien oder in der Ukraine nach dem Vorbild von Nuske u. Nieschulze (2004) würden vermutlich noch größere Lücken ergeben. Dieses Inventurverfahren hätte als Vollaufnahme den Vorteil gegenüber der hier durchgeführten Linientransekt-Stichprobe, die Dichte der Lücken bestimmen zu können und dadurch Vergleiche mit Schirmschlagstellungen zu ermöglichen.

Lücken erweitern sich in den meisten Fällen. Während ein Fünftel aller abgestorbenen Bäume eine Lücke neu bildete, starben vier Fünftel der Bäume im Zuge von Lückenerweiterungen ab. Dieses Ergebnis steht im Gegensatz zu einer Untersuchung von Tabaku u. Meyer (1999), die von einem deutlich geringeren Anteil an Lückenerweiterungen ausgehen. Neue Lücken werden meistens durch den Tod eines einzelnen Baumes verursacht (siehe Abb. 23). Ein Viertel der neu gebildeten Lücken sind durch das Absterben von zwei oder drei Bäumen entstanden. Insgesamt ist der Anteil der Bäume, die eine Lücke neu bildeten, aber gering. Der Lückenanteil betrug in den untersuchten Reservaten 15 bzw. $16 \%$, wenn man Lücken und geschlossenen Bestand durch die überschirmte Fläche von Bäumen aus der Oberschicht unterscheidet (siehe 4.4.1). Unterscheidet man dagegen Lücken und geschlossenen Bestand durch die überschirmte Fläche von Bäumen mit mindestens $7 \mathrm{~cm}$ BHD, beträgt der Lückenanteil 7 bzw. $8 \%$. Entsprechend dieser Lückendefinition ermittelten Tabaku u. Meyer (1999) einen Lückenanteil von 3,3 bis 6,6 \% in albanischen Buchenurwäldern. Zeibig et al. 
(2005) schieden Lücken aus, wenn das Kronendach auf halber Höhe der Bestandeshöhe unterbrochen war, und ermittelten in einem slowenischen Buchenurwald 5,6 \%.

Die Beobachtungen von einem heterogener aufgebauten Baumbestand auf kleiner Fläche im Urwald im Vergleich zum einaltrigem Wirtschaftswald (Korpel' 1995 und Tabaku 1999) fanden sich bestätigt. Die Unterschiede im BHD benachbarter Bäume waren im Urwald deutlich größer als im Altersklassenwald. Die Durchmesserdifferenzierung betrug im Urwald 46,3 bzw. 49,4 \% und im Alterklassenwald 16,8 bis 40,9\% (siehe 3.5.3). Eine vergleichbare Durchmesserdifferenzierung wie im Urwald wurde in Plenterwäldern erreicht: Sie betrug 44,2 bzw. 46,9 \%. Die Verteilung der Baumgruppengrößen im Abschnitt 3.5.1 zeigte ebenfalls kaum Unterschiede zwischen Plenter- und Urwaldbeständen und bestätigte die Ergebnisse der Durchmesserdifferenzierung. Die Plenterbestände wiesen außerdem einen ähnlichen SCI auf wie in Kyjov (siehe 3.5.4), während dieser in Havešová aufgrund höherer Bäume deutlich größer war.

Die Anzahl von Gruppen aus Bäumen mit ähnlicher Größe nahm bei zunehmender Gruppengröße schnell ab. Die Abnahme wurde sowohl im Urwald als auch im Wirtschaftswald beobachtet (siehe 3.5.1). Im Unterschied zum Altersklassenwald nahm die Gruppenanzahl im Urwald aber schneller ab und war gleichmäßiger über die Durchmesserklassen verteilt. Dieser Unterschied war auch bei der Wahl anderer Durchmesserklassen und bei um 10-20 \% verlängerten Kronenradien als Kriterium für die Nachbarschaft zu beobachten.

Bäume mit mehr als $80 \mathrm{~cm}$ BHD traten nicht nur als einzeln stehende Exemplare auf. In Kyjov standen $43 \%$ und in Havešová $83 \%$ dieser großen Bäume in Gruppen aus zwei bis vier Bäumen. Eine Untersuchung von Meyer et al. (2003) ergab eine Tendenz zu einer geklumpten Verteilung von Altbäumen über $80 \mathrm{~cm}$ BHD, die hier Bestätigung fand. Ferner wurde festgestellt, dass sowohl in Urwäldern als auch in bewirtschafteten Altbeständen Baumpaare mit weniger als $6 \mathrm{~m}$ Abstand geringere Durchmesser aufweisen als im Bestandesdurchschnitt (Abb. 28).

Kleinflächig schwankte der Holzvorrat zwischen 100 und 1000 m³/ha. Ein Holzvorrat von $1000 \mathrm{~m}^{3} /$ ha wurde in Havešová auf einer maximalen Fläche von $2650 \mathrm{~m}^{2}$ festgestellt. Ein sehr geringer Vorrat von $136 \mathrm{~m}^{3} /$ ha stockte auf maximal $2500 \mathrm{~m}^{2}$. In Kyjov wurde ein Holzvorrat von 1000 m³a nur auf 3 einzelnen 12,5 x 12,5 m-Quadraten erreicht. In einem Fall stockte auf $2650 \mathrm{~m}^{2}$ ein sehr geringer Holzvorrat von $104 \mathrm{~m}^{3} / \mathrm{ha}$. Der Holzvorrat überstieg auf keiner 
der 0,4 ha großen Stichprobenflächen 1000 m³/ha. Zu ähnlichen Ergebnissen kamen Commarmot et al. (2005), die für den Urwald Uholka in der Ukraine einen Vorrat von durchschnittlich $770 \mathrm{~m}^{3} \mathrm{ha}^{-1}$ ermittelten, der auf 0,25 ha großen Unterflächen von 421 bis $1042 \mathrm{~m}^{3} \mathrm{ha}^{-1}$ variierte.

(II) Der Totholzvorrat beträgt weniger als $10 \%$ des Gesamtholzvolumens (lebender Bestand und Totholz). - Diese These wurde widerlegt. Im Unterschied zu den albanischen Buchenurwäldern (Tabaku 1999) wurde ein höherer Totholzanteil festgestellt, der 15 bzw. 20 \% beträgt. Tabaku (1999) ermittelte einen Totholzanteil von 3,9 bis 9,6 \%. Commarmot (2005) ermittelte einen Anteil von 13,0 \% und Chernyavskyy (2005) 14,3\%. Der lebende Bestandesvorrat betrug bei Commarmot (2005) 770 m³/ha (Spitzenhöhe: 40,3 m / Grundfläche: 38,5 m²/ha) und bei Chernyavskyy (2005) 471 m²/ha (Höhe: $36 \mathrm{~m} /$ Grundfläche: 35,9).

Im Wirtschaftswald können diese Anteile im Einklang mit anderen wichtigen Bewirtschaftungszielen nicht erreicht werden. Die Forderungen von Ammer (1991) und Müller (2005) von 15 bis $40 \mathrm{~m}^{3}$ Totholz je Hektar entsprechen etwa einem Drittel des Totholzvolumens in Buchenurwäldern. Für verbindliche Zielvorgaben wären allerdings weitere Informationen über den Bedarf an Totholz für Saprophyten und über den Bedarf an Saprophyten für den Wald wünschenswert. Insgesamt scheinen die genannten Forderungen jedoch einen möglichen Rahmen zu bilden. Wenn ein verbindlicher Totholzanteil vorgegeben wird, sollte dies für einzelne Bestände in Abhängigkeit von ihrer Wüchsigkeit geschehen.

(III) Verjüngung ist überall vorhanden. - Diese Hypothese wurde nicht widerlegt. In beiden Reservaten waren 90 \% der Probekreise mit Jungpflanzen besetzt. Ähnliche Ergebnisse liegen aus albanischen Urwäldern vor, in denen 84 bis $96 \%$ der Probekreise $\left(10 \mathrm{~m}^{2}\right)$ besetzt waren (Tabaku 1999). Die Pflanzendichte war jedoch zum Teil gering. Während im Durchschnitt 65.000 bzw. 77.000 Jungpflanzen pro Hektar vorkamen, lag der Median bei 29.000 bzw. 51.000 Pflanzen/ha. Korpel' (1995) stellte in Havešová auf drei jeweils 0,5 ha großen Flächen 6.000 bis $40.000 \mathrm{Pfl} . /$ ha fest. In albanischen Urwäldern betrug die Pflanzendichte weniger als 30.000 Pfl./ha (Tabaku 1999).

Im Plenterwald war die Pflanzendichte dagegen größer. So kamen rd. 124.000 Pfl./ha im Plenterwald Bleicherode vor (Tabaku 1999). In einem anderen plenterartigen Bestand wurden rd. 87.000 Pfl./ha festgestellt (Drößler 2001). 
Der Anflug von Samen anderer Baumarten erfolgte ebenfalls auf der ganzen Fläche. So wurden zumindest auf allen Stichprobenflächen $(62,5 \mathrm{~m}$ x 62,5 m) andere Baumarten neben der Buche gefunden. Die Dichte variierte aber deutlich stärker als bei Buche. Höhen über $1 \mathrm{~m}$ wurden von diesen Arten kaum erreicht. Unter $50 \mathrm{~cm}$ Pflanzenhöhe lag ihr Anteil in Havešová bei rd. 50 \% und in Kyjov bei rd. 10 \%. Durch die sehr geringe Anzahl größerer Pflanzen erscheint die Konkurrenzfähigkeit der beteiligten Mischbaumarten unter den ökologischen Bedingungen im Buchenurwald gering.

(IV) Die ökologischen Bedingungen für das Wachstum der Buchen in der Slowakei sind mit deutschen Buchenbeständen vergleichbar. - Diese Hypothese wurde bestätigt. Die Buche besitzt eine weite Standortamplitude, wie auch das Ökogramm aus Leuschner (1997) zeigt. pH-Werte im Boden können 3 bis über 7 betragen und spielen keine entscheidende Rolle (Leuschner 1998). Die Humusform (Rohhumus bis Mull) und das Nährstoffangebot begrenzen die Buche in ihrem Vorkommen ebenfalls nicht (Leuschner et al. 1993). Ihr Wuchsoptimum findet sie auf „tiefgründigen, nachhaltig frischen, basenreichen, gut durchlüfteten und drainierten Braunerden“ (Mayer 1984, zit. aus Czajkowski et al. 2006). Anders als der Boden besitzt das Klima einen großen Einfluss auf das Vorkommen der Buche: Lange, strenge Winter und Sommertrockenheit begrenzen ihre Verbreitung im Osten (Tarasiuk 1999). De Candolle (1855) und Willkomm (1887, beide zit. aus Czajkowski et al. 2006) heben die Bedeutung der durchschnittlichen Wintertemperatur über $-6{ }^{\circ} \mathrm{C}$ und der Niederschlagsmenge in der Vegetationsperiode hervor. Ellenberg (1996) leitet dagegen aus dem Verhältnis der mittleren Julitemperatur und dem Jahresniederschlag einen Weiser ab. Niederschläge über $450 \mathrm{~mm}$ in der Vegetationsperiode stellen keinen Limitierungsfaktor dar. In Uzhgorod ( $25 \mathrm{~km}$ Luftlinie von den Reservaten entfernt, auf $118 \mathrm{~m}$ ü. NN) beträgt die durchschnittliche Temperatur im Januar $-3^{\circ} \mathrm{C}$ und im Juli $20^{\circ} \mathrm{C}$ (Mühr 2002). Im Frühjahr sind Spätfröste die größte Gefahr für Keimlinge und Blüten (Röhrig et al. 2006). Spätfrostschäden konnten aber in beiden Reservaten nicht entdeckt werden. Die vermutete höhere Spätfrostgefahr im Osten aufgrund eines kontinentaleren Klimas wird durch die Lage in den Bergen verringert, in denen die Kaltluft abfließen kann. Dieses Phänomen ist z.B. im Klimaatlas für Polen gut dokumentiert (Kozminski u. Michalska 2001). Der frühere Blattaustrieb der Buchen aus Havešová im Vergleich zu Buchen aus dem Solling kann als Indikator für eine Anpassung an eine geringe Spätfrostgefahr interpretiert werden. In einem Vergleich der Frostresistenz von Buchen entlang eines Nord-Süd-Gradienten beschreiben 
Višnjic u. Dohrenbusch (2002) eine rumänische Herkunft, die im Vergleich zu einer Herkunft aus dem Solling 11 Tage früher austrieb.

Insgesamt zeigten die Untersuchungsbestände eine sehr gute Höhenwuchsleistung und eine große Konkurrenzkraft gegenüber anderen Baumarten. Deshalb kann man davon ausgehen, dass das im Vergleich zu Deutschland kontinentaler geprägte subozeanische Klima keine großen Unterschiede im Wachstum und im Bestandesaufbau im Vergleich zu Buchenwäldern in Deutschland hervorruft. Diese Annahme wird auch dadurch gestützt, dass Buchenurwälder auf unterschiedlichen Standorten, z.B. in Albanien (Tabaku 1999, Tabaku u. Meyer 1999), Slowenien (Zeibig et al. 2005), in der Slowakei (Korpel' 1995) oder Ukraine (Commarmot et al. 2005, Chernyavskyy 2005), ähnliche Bestockungsverhältnisse und Lückenmuster aufweisen.

Die genetischen Untersuchungen geben ebenfalls keine Hinweise darauf, dass zwischen Havešová und deutschen Buchenbeständen große Unterschiede bestehen (Konnert et al. 2000). Während sich das genetische Material von Bucheckern norddeutscher Bestände untereinander um 3 bis $6 \%$ unterscheidet, liegen die Unterschiede zum Reservat Havešová zwischen zwischen 6 und $11 \%$ (siehe 2.3.5).

Die Artenzusammensetzung in der Krautschicht der untersuchten Reservate ähnelt den Buchenwaldgesellschaften des Galio odorati-Fagenions in Deutschland. So konnten in Havešová elf Arten bestimmt werden, die auch durch eine Aufnahme im Habichtswald bei Kassel von Hakes (1991, zit. nach Ellenberg 1996) beschrieben werden. Lediglich vier der 26 vorgefundenen Arten wurden nicht in dem Werk ,Zeigerwerte von Pflanzen in Mitteleuropa“ von Ellenberg (2001) aufgeführt. Die Zeigerwerte für diese Arten wurden von Zolyomi et al. (1966) angegeben. Insgesamt ergab sich im Habichtswald eine mittlere Feuchtezahl von 5,3 im Vergleich zu 5,5 in Havešová und 5,6 in Kyjov. Die Stickstoffzahl betrug 5,9 (in Hav. 6,0 u. in Kyj. 5,8), die Lichtzahl 3,6 (in Hav. 3,2 u. in Kyj. 4,1), die Temperaturzahl 5,1 (in Hav. 4,9 u. in Kyj. 4,3) und die Kontinentalitätszahl 3,5 (in Hav. 3,9 u. Kyj. 3,8).

(V) Waldentwicklungsphasen treten meistens deutlich kleinflächiger auf, als auf den von Korpel' (1995) ausgewiesenen Untersuchungsflächen. - Diese Hypothese wurde bestätigt. Die Gruppierung von Bäumen ähnlicher Dimension (siehe 3.5.1 u. 5.5.2) und die vorgefundenen Lückengrößen im Abschnitt 4.5.2 demonstrieren, dass Waldentwicklungsphasen unterschiedlich große Flächen einnehmen können. Kleine Flächen werden deutlich häufiger von einzelnen Phasen eingenommen als große Flächen. Ein einzelner Baum kann bereits eine Waldentwicklungsphase darstellen. Ältere Phasen können 
sich mit jüngeren Phasen überlappen. Diese Beobachtung bestätigt das Ergebnis von Grassi (2003) in einem ungleichaltrigen Plenterwald aus Nadelbäumen in den Alpen.

Die Vermutung, dass eine Waldentwicklungsphase im Extremfall eine Fläche von 1/2 ha einnehmen kann, konnte nicht widerlegt werden. Die größte Fläche einer Phase betrug auf den 0,4 ha großen Stichprobenflächen 0,25 ha. Das gewählte Stichprobendesign war für diese Fragestellung ungeeignet. Allerdings lässt die maximale Lückengröße als eine Schlüsselgröße für die Waldentwicklung die Vermutung zu, dass 0,5 ha große Flächen die maximale Ausdehnung von Waldentwicklungsphasen darstellen können. Korpel' (1995) wählte vor 50 Jahren 0,5 ha große Untersuchungsflächen, die er nach Waldentwicklungsphasen unterschied. Heute kann man auf diesen Flächen allerdings wieder mehrere Phasen unterscheiden.

\subsection{Waldbauliche Schlussfolgerungen}

Natürliche Störungen lassen sich in Buchenwäldern am besten durch einzelstammweise Nutzungen über einen langen Zeitraum und gelegentliche Loch- oder Femelhiebe simulieren. Im Gegensatz zum klassischen Femelschlagverfahren (beschrieben durch Röhrig et al. 2006) sollten Eingriffe aber so erfolgen, dass die Verjüngung zu keiner Zeit großflächig aufläuft. Die kleinflächig heterogene Struktur im Urwald wird in erster Linie durch die Ungleichaltrigkeit der Bäume verursacht.

Ein Anteil von $65 \%$ der Einzelbaumlücken an der Lückenzahl bescheinigt dem Plenterschlagverfahren eine sehr naturnahe Vorgehensweise. Interessant ist aber auch, dass die Hälfte der Gesamtlückenfläche von Lücken mit mehr als $600 \mathrm{~m}^{2}$ Größe eingenommen wird. Diese Störungen können durch vereinzelte Femelschläge simuliert werden. Weiterhin zeigt die Untersuchung, dass in sehr seltenen Fällen (< $1 \%$ der Lücken) großflächige Störungen im Urwald vorkommen.

Wenn bereits vor der Endnutzung auf besonders naturnahe Strukturen hingearbeitet werden soll, kann im Rahmen von Durchforstungen auf eine große Durchmesserdifferenzierung benachbarter Bäume geachtet werden. Dazu demonstriert Tabelle 16, welche Gruppengrößen in den unterschiedlichen Alterstufen auftreten. So zeigt sich z.B. in der BHD-Klasse von 7-15 cm, dass rund ein Zehntel der Bäume in Gruppen aus mehr als 20 Bäumen stehen können. Bäume dieser Größe entsprechen im Wirtschaftswald der Alterstufe des Gertenholzes. Der geringe Anteil von 0,5 \% an Mischbaumarten lässt vermuten, dass das kleinflächige Störungsregime im Urwald nicht ausreicht, um lichtbedürftigere Arten in nennenswerten Anteilen ohne weitere Pflegeeingriffe zu erhalten. In diesem Fall würde das Ziel Naturnähe dem Ziel Artenvielfalt entgegenstehen. 
Der entscheidende Unterschied des Wirtschaftswaldes zum Urwald liegt in der fehlenden Zerfalls- und Terminalphase. Eine Annäherung kann durch entsprechendes Totholzmanagement und dem Belassen von Altholzgruppen versucht werden. Totholzanteile von 10-20\% wie im Urwald können aber im Einklang mit anderen wichtigen Bewirtschaftungszielen nicht erreicht werden. Zielvorgaben für den Totholzanteil sollten sich außerdem an der Wüchsigkeit eines Bestandes orientieren. Das Erreichen der größten Stammdurchmesser ist im Wirtschaftswald ebenfalls problematisch, weil diese Bäume durch innere Stammfäule stark entwertet werden.

\section{Zusammenfassung der Dissertation}

\section{Zielsetzung}

Die Arbeit beschreibt den Waldaufbau (waldwachstumskundliche Kennwerte, Waldentwicklungsphasen, Baumgruppen) und das Störungsregime (Größe und Neubildungsrate von Lücken) von Buchenurwäldern am Beispiel von zwei Reservaten im Osten der Slowakei. Weiterhin wurde die Vergleichbarkeit mit deutschen Beständen anhand ökologischer Parameter (Vegetation, Boden, Klima, Verjüngung, Strahlung am Boden, genetische Merkmale) beurteilt.

\section{Untersuchungsansatz}

Die Untersuchung hat zwei Ansätze verfolgt. In beiden Fällen handelt es sich um Stichproben. Der erste Ansatz umfasst systematische Stichprobenflächen, die gleichmäßig verteilt in jedem Reservat angelegt wurden. Auf diesen Flächen wurden waldwachtumskundliche Kennwerte (Stammzahl, Grundfläche, Holzvorrat, Totholzvorrat etc.), Waldentwicklungsphasen und Baumgruppen erfasst. Der zweite Ansatz beruht auf Linientransekt-Stichproben, die zur Untersuchung der Bestandeslücken genutzt wurden. Über den Anteil der Bestandeslücken konnte die Lückenbildungsrate und der durchschnittliche Turnus einer Waldgeneration geschätzt werden. Die auf dem Transekt erfassten Lücken wurden mit den abgestorbenen Bäumen vermessen und die Entstehungsgeschichte jeder Lücke nachträglich konstruiert.

\section{Ergebnisse}

In Havešová stockte ein durchschnittlicher Holzvorrat von 720 m³/ha, in Kyjov von 450 $\mathrm{m}^{3} / \mathrm{ha}$. Kleinflächig schwankten die Werte zwischen 100 und $1000 \mathrm{~m}$ 3/ha. Das Totholzvolumen betrug $130 \mathrm{~m}^{3} / \mathrm{ha}$ bzw. $110 \mathrm{~m}^{3} / \mathrm{ha}$, was einem Fünftel des lebenden Vorrats entspricht. In beiden Reservaten standen ungefähr 300 Bäume mit mindestens $7 \mathrm{~cm}$ BHD auf 
einem Hektar. Die Buche bildete auf beiden Standorten Reinbestände mit einem Mischbaumartenanteil von 0,5\%. Die Grundfläche betrug in Havešová $35 \mathrm{~m}^{2} / \mathrm{ha}$ und in Kyjov 32 m²/ha. Zusammenhänge zwischen Bestandesdichte und Bestandesstruktur und zwischen Bestandesdichte und Stammqualität konnten nicht festgestellt werden.

Das Wasser- und Nährstoffangebot im Boden, die Zusammensetzung der Krautvegetation, die Dichte der Verjüngung, die Strahlungsverhältnisse am Boden und die genetische Ausstattung ergaben keine großen Unterschiede im Vergleich zu bewirtschafteten Buchenbeständen in Deutschland.

Die Ausscheidung von Waldentwicklungsphasen ergab in beiden Urwäldern einen Anteil von 70 Prozent der Plenter-, Terminal- und Zerfallsphase. Die Optimalphase nahm ungefähr 15 Prozent ein. Diese Verteilung unterscheidet sich deutlich vom Wirtschaftswald, in dem die Optimalphase bei weitem überwiegt.

Zwischen den einzelnen Waldentwicklungsphasen schwankte das Holzvolumen von 100 $\mathrm{m}^{3} / \mathrm{ha}$ bis $1000 \mathrm{~m}$ /ha und die Stammzahl von 30 bis $400 \mathrm{Stück} / \mathrm{ha}$. Das Totholzvolumen reichte in Havešová von 14 bis 490 m³/ha und in Kyjov von 7 bis 320 m³ha.

Die Gruppierung von Bäumen ähnlicher Größe ergab einen sehr kleinflächigen Wechsel unterschiedlich großer Bäume im Urwald, der in diesem Merkmal mit einem Plenterwald vergleichbar ist. Die Anzahl der Baumgruppen nimmt mit zunehmender Gruppengröße exponentiell schnell ab. Am häufigsten waren einzeln stehende Bäume. Die größten Baumgruppen im Urwald setzten sich in der BHD-Klasse von 7-15 cm aus 36 Bäumen, in der Klasse 21-34 cm aus 20 Bäumen und in der Klasse 65-79 cm aus 17 Bäumen zusammen. Das letzte Beispiel überschirmte bereits 0,2 ha Fläche. Diese auf die Gruppierung von Bäumen ähnlicher Dimension bezogene Analyse der Bestandesstruktur führte zu Ergebnissen, die leicht vorstellbar sind und deshalb im Wirtschaftwald nachgeahmt werden können, falls ein besonders naturnaher Waldaufbau das Ziel ist. Die Analyse kann unabhängig von der Struktur unterschiedlich bewirtschafteter Waldbestände durchgeführt werden.

Die Berechnung verschiedener Strukturindices (Durchmesserverteilung, Durchmesserdifferenzierung, ein struktureller Komplexitätsindex, Paar- und Markenkorrelationsfunktionen) ergab dagegen oft nur ein relatives Ranking zwischen unterschiedlich naturnahen Wäldern. Lediglich Durchmesserverteilungen ließen praxisrelevante Handlungsoptionen erkennen, die noch dazu mit wesentlich geringerem Aufwand erstellt werden können. 
Ähnlich wie die Häufigkeit der Gruppen aus Bäumen einer BHD-Klasse nahm auch die Häufigkeit der Bestandeslücken mit zunehmender Größe ab. 65 \% der Lücken (= Unterbrechung der oberen Bestandesschicht) wurden durch den Tod eines Baumes verursacht, 20 \% durch den Tod von 2,3 oder 4 Bäumen. Die Kronendachöffnung der größten Lücke war in beiden Reservaten jeweils 0,4 ha groß, in Havešová waren dort 56 Bäume und in Kyjov 80 Bäume der Oberschicht in den letzten 40 Jahren abgestorben. Der Anteil so einer großen Lücke an der Gesamtlückenzahl war kleiner als $1 \%$. Insgesamt bildeten ein Fünftel der abgestorbenen Bäume eine neue Lücke, während vier Fünftel bestehende Lücken erweiterten. Der Lückenanteil betrug 15 bzw. $16 \%$ der Waldfläche. In Havešová wurde Windwurf als häufigste Absterbeursache festgestellt, in Kyjov dagegen Windbruch.

Der Anteil des Totholzvorrats am Gesamtholzvorrat (lebender Bestand und Totholz) schwankte im Urwald zwischen 10 und $20 \%$. Im Einklang mit anderen wichtigen Zielen können diese Anteile im Wirtschaftswald nicht erreicht werden. Derzeitige Forderungen entsprechen etwa einem Drittel dieses Totholzanteils und geben wahrscheinlich einen möglichen Kompromiß wieder.

Natürliche Störungen lassen sich in Buchenwäldern am besten durch einzelstammweise Nutzungen über lange Zeiträume und Loch- bzw. Femelhiebe simulieren. In sehr seltenen Fällen können auch großflächigere Störungen natürliche Prozesse nachbilden. 


\section{Literatur zur Einleitung und Diskussion}

(Literaturquellen, die man hier nicht findet, sucht man am Ende der entsprechenden Publikation.)

Ammer, U.: Konsequenzen aus den Ergebnissen der Totholzforschung für die forstliche Praxis. Forstw. Cbl. 110: 149-157.

Bohn, U. und Weber, H. 2000. Karte der natürlichen Vegetation Europas. Bundesamt für Naturschutz, Bonn. Cermak, L. 1910. Einiges über den Urwald von waldbaulichen Gesichtspunkten. Cbl. ges. Forstwes. 36: 340370.

Chernyavskyy, M. 2005. The dynamics of virgin beech forests in the Ukrainian Carpathians. In: Hamor, F.D. and Commarmot, B. (eds): Natural forests in the temperate zone of Europe - Values and utilisation. International conference in Mukachevo, Ukraine. October 13-17, 2003. Rakhiv, Carpathian biosphere Reserve; Birmensdorf, Swiss Federal Research Institute WSL.

Clark, P. J., Evans, F. C. 1954. Distance to nearest neighbour as a measure of spatial relationships in populations. Ecology 35: 445-453.

Commarmot, B. 2005. Structures of virgin and managed beech forests in Uholka (Ukraine) and Sihlwald (Switzerland): a comparative study. For. Snow Landsc. Res. 79, 1/2: 45-56.

De Candolle, A.: Geographie Botanique raisonée. Paris et Genévé, 1855

Dengler, A. und Markgraf, F. 1931. Aus den südosteuropäischen Urwäldern. I. Die Wälder Albaniens. II. Die Ergebnisse einer Probeflächenaufnahme im Buchenurwald Albaniens. Zeitschrift f. Forst- u. Jagdwesen 63: 2032 u. 310-319.

Donelly, K. P. 1978. Simulations to determine the variance and edge effect of total nearest-neighbour distances. In: Simulation Methods in Archaeology. Cambridge Univ. Press. London.

Drößler, L. 2001. Untersuchungen zum Aufbau eines plenterartigen Laubholzbestandes mit hohem Edellaubholzanteil. Master thesis Univ. Göttingen. (unveröffl.)

Ellenberg, H., 1996. Vegetation Mitteleuropas mit den Alpen. Ulmer Verlag. Stuttgart.

Ellenberg, H., 2001. Zeigerwerte von Pflanzen in Mitteleuropa. Goltze Verlag. Göttingen.

Fröhlich, J. (1925): Aus dem südosteuropäischen Urwalde. Fw. Cbl. 47:199-220.

Füldner, K. 1995. Strukturbeschreibung von Buchen-Edellaubholz-Mischwäldern. Diss. Univ. Göttingen.

Cuvillier. Göttingen.

Gehrhardt, 1923. Ueber Urwaldungen in den Karpathen. Forstliche Wochenschrift Silva 1:361-363.

Gleichmar, W. and Gerold, D. 1998. Indizes zur Charakterisierung der horizontalen Baumverteilung. Ger. J. For. Sci. 117: 69-80.

Grassi, G., Minotta, G., Giannini, R., Bagnaresi, U. 2003. The structural dynamics of managed uneven-aged conifer stands in the Italian eastern Alps. For. Ecol. Manage. 185: 225-237.

Hakes, W. 1991. Das Galio odorati-Fagenion im Habicgtswald bei Kassel - Untersuchungen zur ökologischen Feingliederung. Tuexenia 11: 381-406.

Konnert, M., Ziehe, M., Tröber, U., Maurer, W., Janßen, A., Sander, T., Hussendörfer, E., Hertel, H., 2000. Genetische Variation der Buche (Fagus sylvatica L.) in Deutschland: Gemeinsame Auswertung genetischer Inventuren über verschiedene Bundesländer. Forst und Holz 55, 403-408.

Korpel', Š. 1967. Vyvoj a vekova struktura bukoveho Pralesa na vyhorlate. (Aufbau und Alterstruktur des Buchenurwaldes im Vyhorlatgebirge.) Biologia 22(4): 285-303. Slowakisch.

Korpel', Š. 1995. Die Urwälder der Westkarpaten. Gustav Fischer Verlag, Stuttgart, Jena, New York.

Kozminski, C., Michalska, B. 2001. Atlas klimaticznego ryzyka uprawy roslin w Polsce. (Atlas der klimatischen Risiken für den Ackerbau in Polen.) Uniwersytet Szczecinski, Szczecin.

Leibundgut, H. 1993. Europäische Urwälder. Verlag Paul Haupt, Bern, Stuttgart.

Le Tacon, F.: Caracterisation edaphique. In : Teissier du Cros, E. La Hêtre. 77-84, INRA, Paris, 1981

Leuschner, C., Rode, M., Heinken, T.: Gibt es eine Nährstoffmangel-Grenze der Buche im nordwestdeutschen Flachland? Flora 188: 239-249, 1993

Leuschner, C.: Das Konzept der potentiellen natürlichen Vegetation (PNV): Schwachstellen und Entwicklungsperspektiven. Flora 192: 379-391, 1997

Leuschner, C.: Mechanismen der Konkurrenzüberlegenheit der Rotbuche. Ber. d. Reinh.-Tüxen-Ges. 10: 5-18, 1998

Mayer, H.: Waldbau auf soziologisch-ökologischer Grundlage. Fischer Verlag, Stuttgart, New York, 1984

Meyer, P., Tabaku, V., Lüpke, B.v., 2003. Die Struktur albanischer Rotbuchen-Urwälder - Ableitungen für eine naturnahe Buchenwirtschaft. Forstw. Cbl. 122: 47-58.

Meyer, P., Ackermann, J., Balcar, P., Boddenberg, J., Detsch, R., Förster, B., Fuchs, H., Hoffmann, B., Keitel, W., Kölbel, M., Köthke, C., Koss, H., Unkrig, W., Weber, J., Willig, J. 2001. Untersuchung der Waldstruktur und ihrer Dynamik in Naturwaldreservaten. IHW-Verlag. Eching.

Mühr, B.: URL http://www.klimadiagramme.de/Europa/uzgorod.html (abgerufen am 29.5.06), 2002

Müller, J.: Waldstrukturen als Steuergröße für Artengemeinschaften in kollinen bis submontanen

Buchenwäldern. Diss. TU München, 2005 
Nagel, J. 2003. BwinPro - Programm zur Bestandesanalyse und Prognose. Version 6.2. URL:

http://www.nfv.gwdg.de/A/BwinPro/Download/bwman60.pdf (28.3.2006).

Neumann, M. 1979. Bestandesstruktur und Entwicklungsdynamik im Urwald Rothwald/NÖ und im Urwald

Čorkova Uvala/Kroatien. Diss. Univ. f. Bodenkultur, Wien.

Nuske, R. and Nieschulze, J. (2004): Die Vegetationshöhe als Werkzeug zur Ermittlung von Bestandeshöhen.

Eine Anwendung automatisierter digitaler Photogrammetrie in der Forstwissenschaft. Allg. Forst- u. J.-Ztg.

175(1/2): 13-21.

Ohser, J., Mücklich, F. 2000. Statistical analysis of microstructures in material science. J. Wiley. Chichester.

Pommerening, A., Biber, P., Stoyan, D., Pretzsch, H. 2000. Neue Methoden zur Analyse und Charakterisierung von Bestandesstrukturen. Forstw. Cbl. 119: 62-78.

Pommerening, A. 2002. Approaches to quantifying forest structures. Forestry, Vol. 75: 305-324.

Pretzsch, H. 2001. Modellierung des Waldwachstums. Parey. Berlin.

Ripley, B.D. 1981. Spatial Statistics. J. Wiley. New York.

Röhrig, E., Bartsch, N.: Waldbau auf ökologischer Grundlage. Parey Verlag, Hamburg, Berlin, 1992

Rubner, K. 1960. Die pflanzengeographischen Grundlagen des Waldbaus. Neumann Verlag, Radebeul, Berlin.

Stoyan, D., Stoyan, H. 1994. Fractals, Random Shapes and Point Fields. Methods of Geometrical Statistics. J.

Wiley. Chichester, New York, Brisbane, Toronto, Singapore.

Tabaku, V. 1999. Struktur von Buchen-Urwäldern in Albanien im Vergleich mit deutschen Buchen-

Naturwaldreservaten und -Wirtschaftwäldern. Göttingen, Univ., Diss.

Tabaku, V., und Meyer, P. (1999): Lückenmuster albanischer und mitteleuropäischer Buchenwälder unterschiedlicher Nutzungsintensität. Forstarchiv, 70: 87-97.

Tarasiuk, S.: Buk zwyczajny (Fagus sylvatica L.) na obrzeżach zasięgu w Polsce. Diss. Univ. Warzsawa, 1999

Tsuryk, J.E. 1980. Struktura i vosobnowlenie devstvennyh bukovyh drevostojev Karpat. Lesovedenie 5: 75-84.

Višnjic, C., Dohrenbusch, A.: Frostresistenz und Phänologie europäischer Buchenprovenienzen (Fagus sylvatica L.). Allg. Forst- u. J.-Ztg., 175: 101-108.

Willkomm, M.: Forstliche Flora von Deutschland und Österreich. Winter Verlag, Leipzig, 1887

Zeibig, A., Diaci, J. und Wagner, S. 2005. Gap disturbance patterns of a beech virgin forest remnant in the mountain vegetation belt of Slovenia. In: Hamor, F.D. and Commarmot, B. (eds): Natural forests in the temperate zone of Europe - Values and utilisation. International conference in Mukachevo, Ukraine. October 1317, 2003. Rakhiv, Carpathian biosphere Reserve; Birmensdorf, Swiss Federal Research Institute WSL.

Zenner, E. K. 2004. Does old-growth condition imply high live-tree structural complexity? For. Ecol. Manage. 195: 243-258.

Zolyomi, B., Barath, Z., Fekete, G., Jakucs, P., Karpati, I., Karpati, V., Kovacs, M., Mate, I., 1966. Einreihung von 1400 Arten der ungarischen Flora in ökologische Gruppen nach TWR-Zahlen. Fragmenta Bot. Mus. Hist. Nat. Hung. 4: 101-142.

\section{Dank}

Ich bedanke mich herzlich bei meinem Betreuer Prof. Dr. Burghard von Lüpke und den Mitarbeitern des Instituts für Waldbau in Göttingen, bei Dr. Peter Jaloviar, Prof. Dr. Saniga, Herrn Fako, Herrn Dudiž, Branislav, Marienka, Palo, Matuš, Jan Dohndorf und meinen lieben Eltern. 
Lebenslauf

Name: $\quad$ Lars Drößler

Geburtsdatum:

12.06.1977

Geburtsort:

Eberswalde-Finow

Familienstand: $\quad$ ledig

Schulausbildung: $\quad$ 1984-1991 Polytechnische Oberschule 15

Suhl

1991-1994 Naturwissenschaftliches Gymnasium

Suhl

1994-1996 Staatliches Gymnasium

Schlotheim

Wehrdienst:

1996-1997 Fernmelderegiment 320

Frankenberg

Studium:

1997-2001 Fakultät für Forstwissenschaften und Waldökologie der Georg-August-Universität Göttingen

Berufstätigkeit:

seit 2001 wiss. Angestellter am Institut für Waldbau der Universität Göttingen 